SLAC -291

$\mathrm{UC}-34 \mathrm{D}$

$(\mathrm{T} / \mathrm{E})$

\title{
SYSTEMATICS OF MESON-SKYRMION SCATTERING *
}

\author{
Michael Perelman Mattis \\ Stanford Linear Accelerator Center \\ Stanford University \\ Stanford, California 94305
}

February 1986

Prepared for the Department of Energy

under contract number DE-AC03-76SF00515

Printed in the United States of America. Available from the National Technical Information Service, U.S. Department of Commerce, 5285 Port Royal Road, Springfield, Virginia 22161. Price: Printed Copy A06, Microfiche A01.

* Ph.D. Dissertation. 


$$
\begin{aligned}
& \mathbf{S}_{L \frac{3}{2}, L-\frac{1}{2}}=\frac{(2 L-1)(L-1)}{6 L(2 L+1)} 8_{L-1, L, L}+\frac{2 L-1}{6 L} 8_{L L L}+\frac{2 L+3}{4 L+2} 8_{L+1, L, L} \\
& \mathbf{S}_{L \frac{s}{2}, L+\frac{1}{2}}=\frac{2 L-1}{4 L+2} 8_{L-1, L, L}+\frac{2 L+3}{6 L+6} 8_{L L L}+\frac{(L+2)(2 L+3)}{6(L+1)(2 L+1)} 8_{L+1, L, L}
\end{aligned}
$$

(Of course (III.21a) and (III.21c) only make sense if $L>0$; likewise the first term on the right-hand side of (III.21d) is absent for $L=0$.) Thus for example the $P_{11}$ channel examined in [21] is given by $(1 / 3) s_{011}+(2 / 3) s_{111}$ instead of pure "breathing mode" sol1 $_{\text {; }}$ we will return to this channel shortly.

The Argand plots obtained from these equations are presented in Figure 19, juxtaposed with the corresponding experimental results as drawn from Höhler et al. ${ }^{|26|}$ (The experimental graphs are the 'inner' ones.) As is customary we have graphed the $T$-matrix instead of the $S$-matrix; they are related by $T=(S-1) / 2 i$. Pion energy $\omega$ is given in units of $e f_{\pi}$ for our graphs, while those drawn from Ref. 24 are parametrized by total center-of-mass energy $W$ in $\mathrm{GeV}$. For each of our resonances we give the corresponding value of $\omega$, as well as the mass and width in $\mathrm{MeV}$, using our 'best-fit' values $\left\{e=4.79, f_{\pi}=150 \mathrm{MeV}, e f_{\pi}=718.5 \mathrm{MeV}\right\}$ that we shall obtain at the end of this section. ${ }^{*}$ The locations of resonances in the real-world data are indicated by vertical lines. Note that, as mentioned earlier, a resonance in the Skyrme model (as determined by the speed criterion) tends to occur toward the right-hand side of a circle and not at the top.

Before discussing the successes of the model we should confront its failures; these lie in the $S$ - and $P$-waves. Indeed one's natural inclination is to turn first to the $P_{33}$ channel, where in one of the cleanest examples of elastic scattering in Nature the delta manifests itself dramatically as a full rotation around the unitarity circle. Instead, one finds in the Skyrme model initial repulsive (i.e., clockwise) behavior, followed by a highly inelastic resonance at $\omega \simeq .34 \mathrm{ef}$, then

* In light of our "no-recoil" approach, which is not only built into our treatment of pion scattering in the fired skyrmion background but also implicit in the derivation of Eq. (II.11), we interpret the rest-mass of a resonance to be given simply by the sum $m_{N}+w$; this is in accord with Ref. 21. 

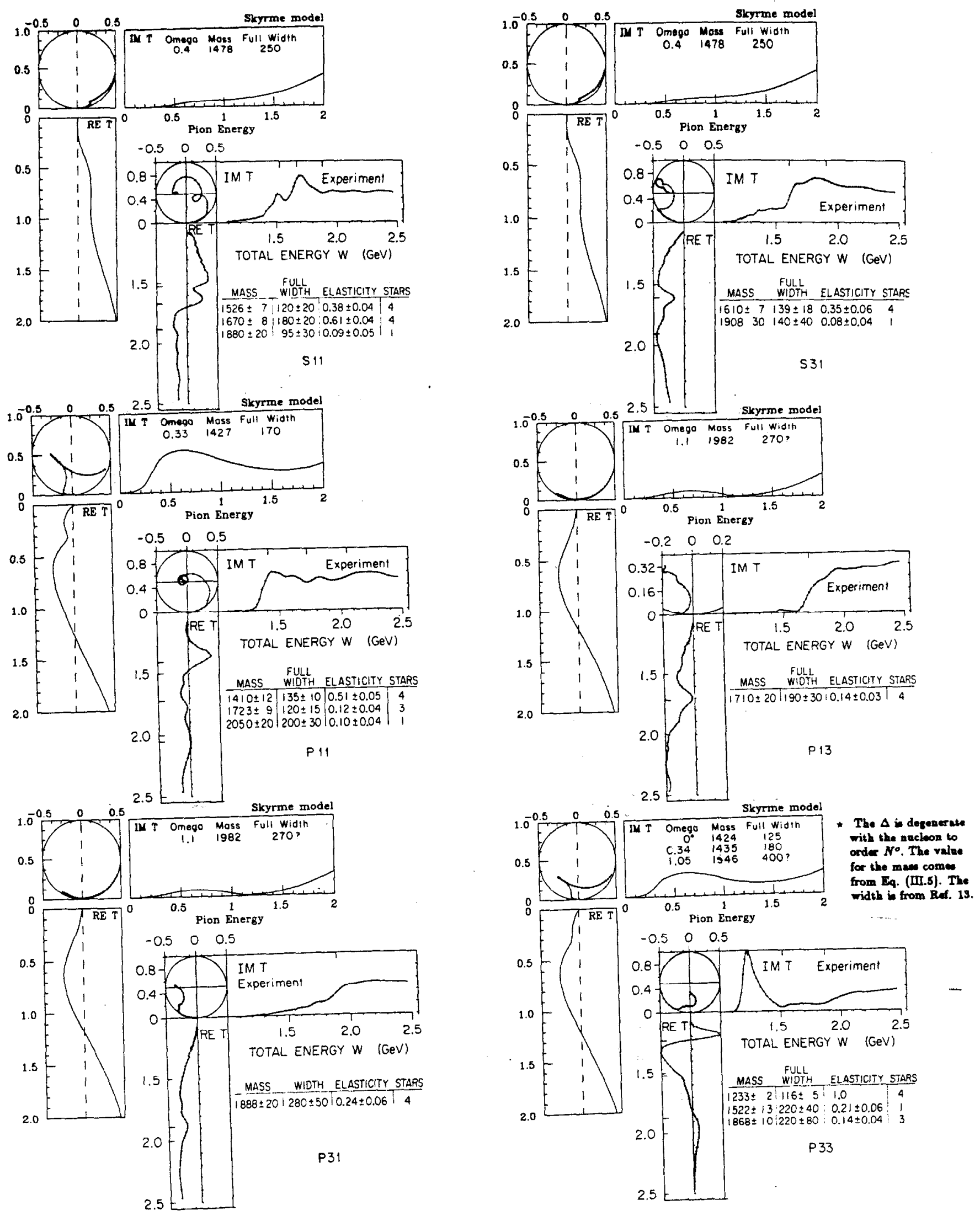

Fig. 19. Partial-wave T-matrix for Skyrme model vs. Nature. The Skyrme-model plots are parametrized by pion energy $w$, in units of $e f_{\pi}$. The experimental graphs are in terms of total c.o.m. energy $W$. 

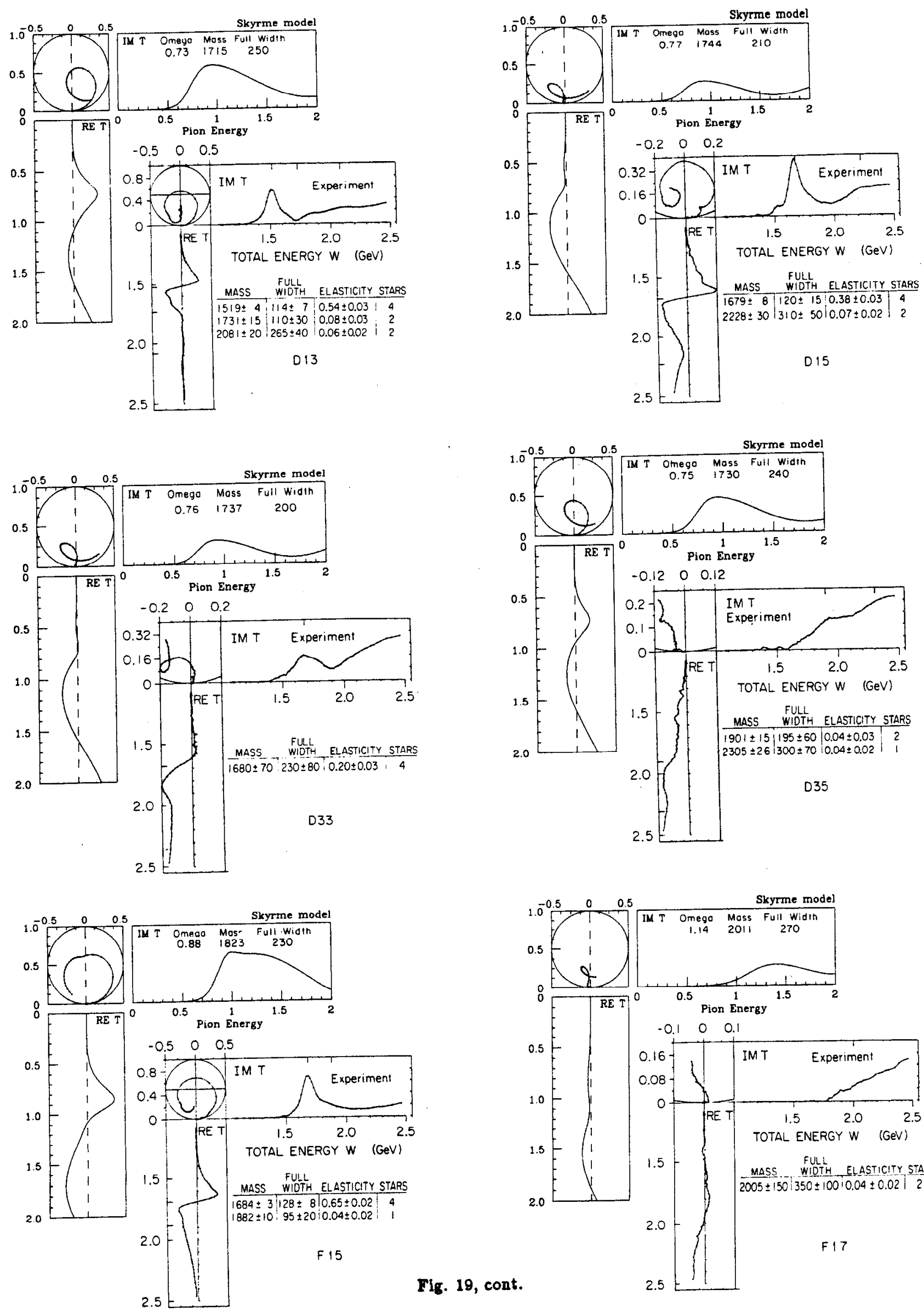

Fig. 19, cont.

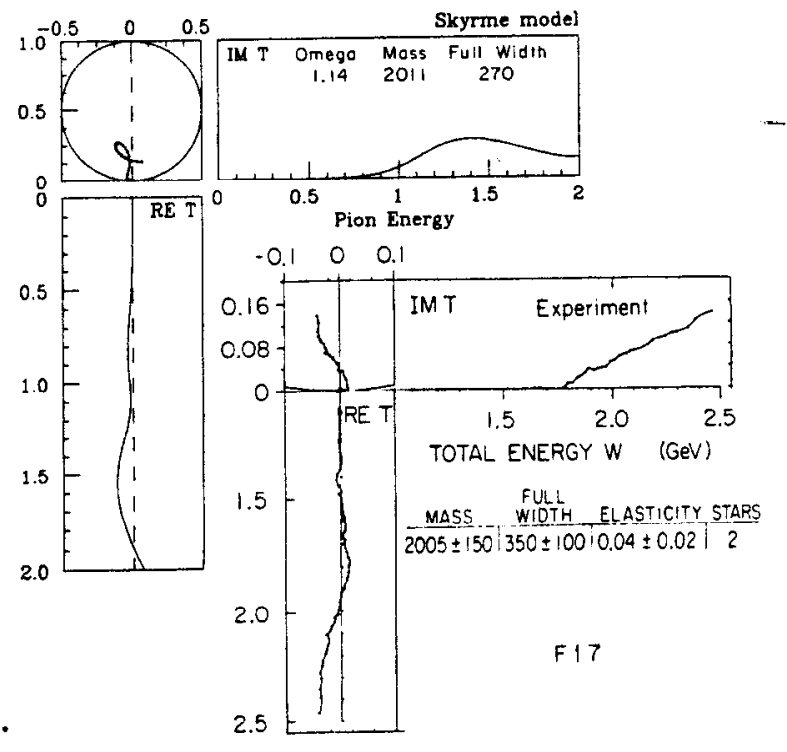



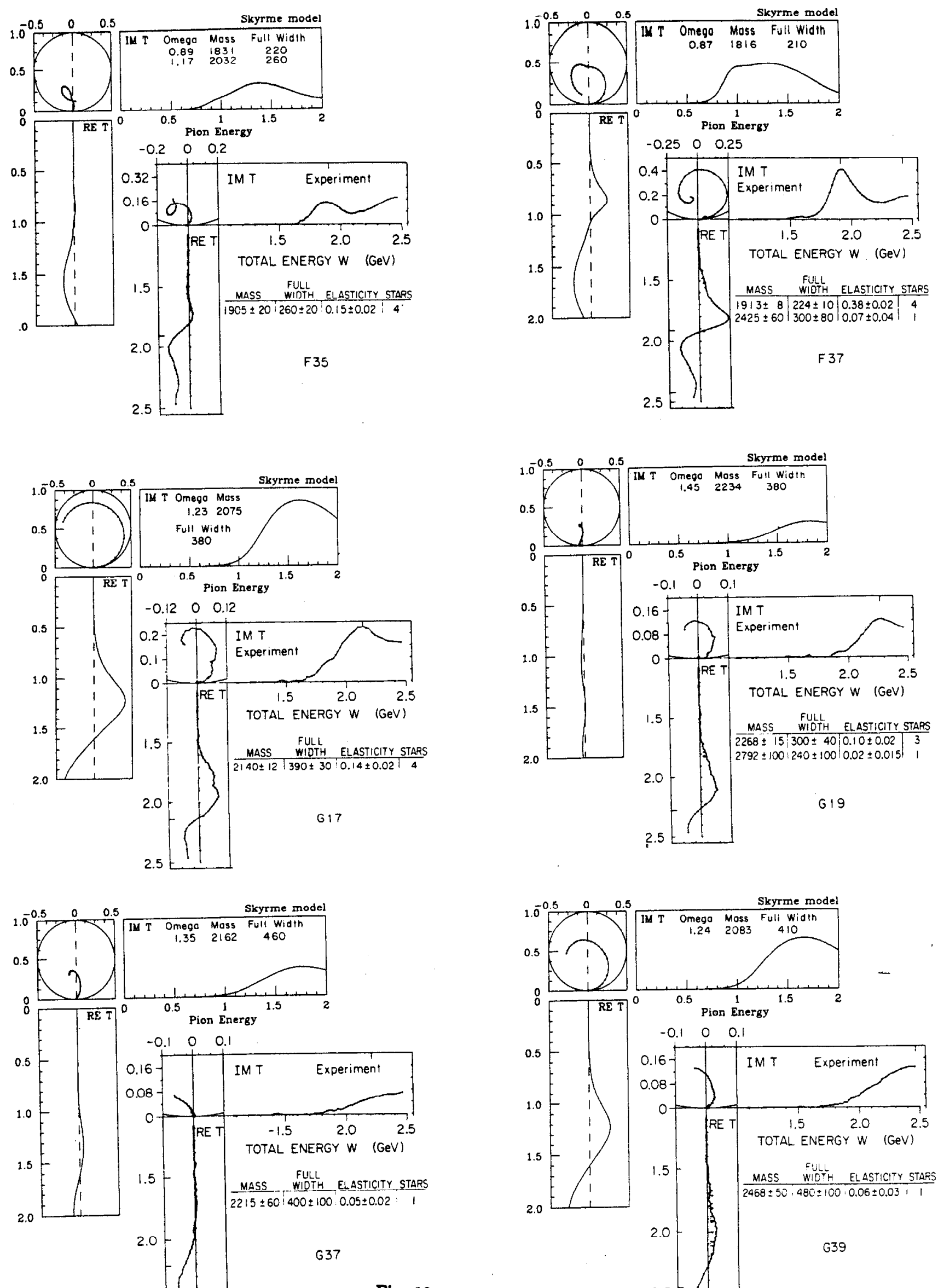

Fig. 19, cont.

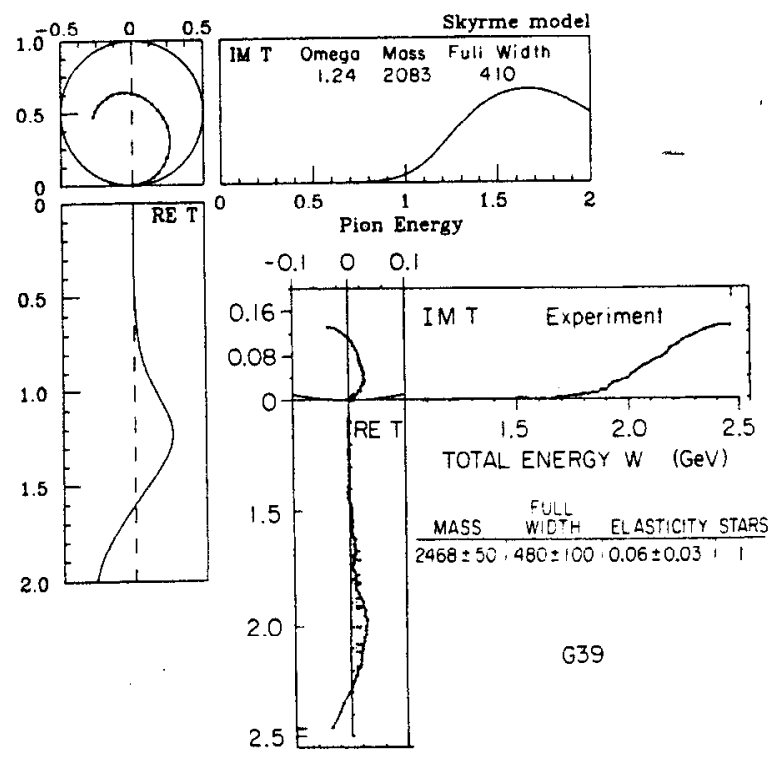



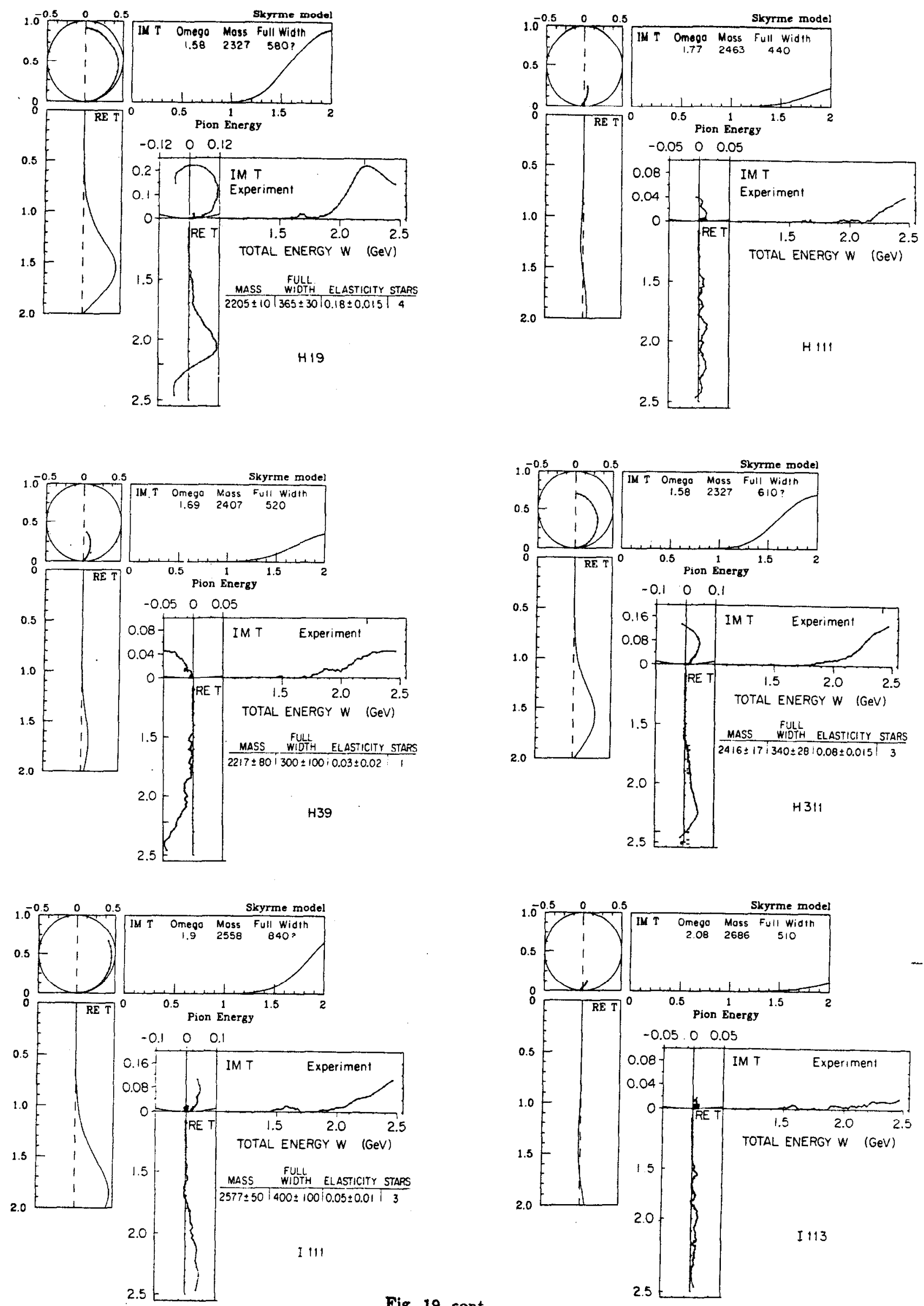

Fig. 19, cont.

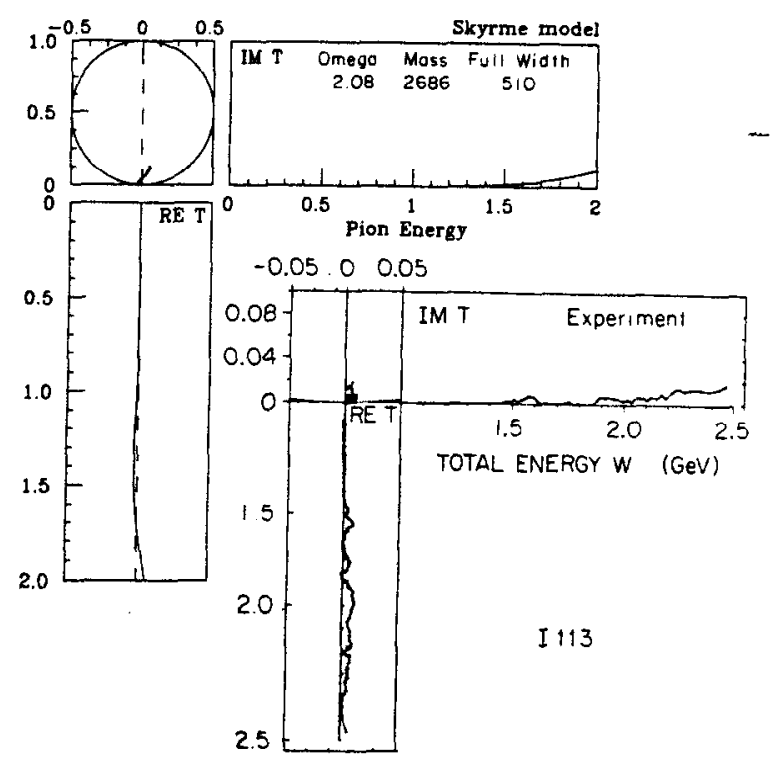



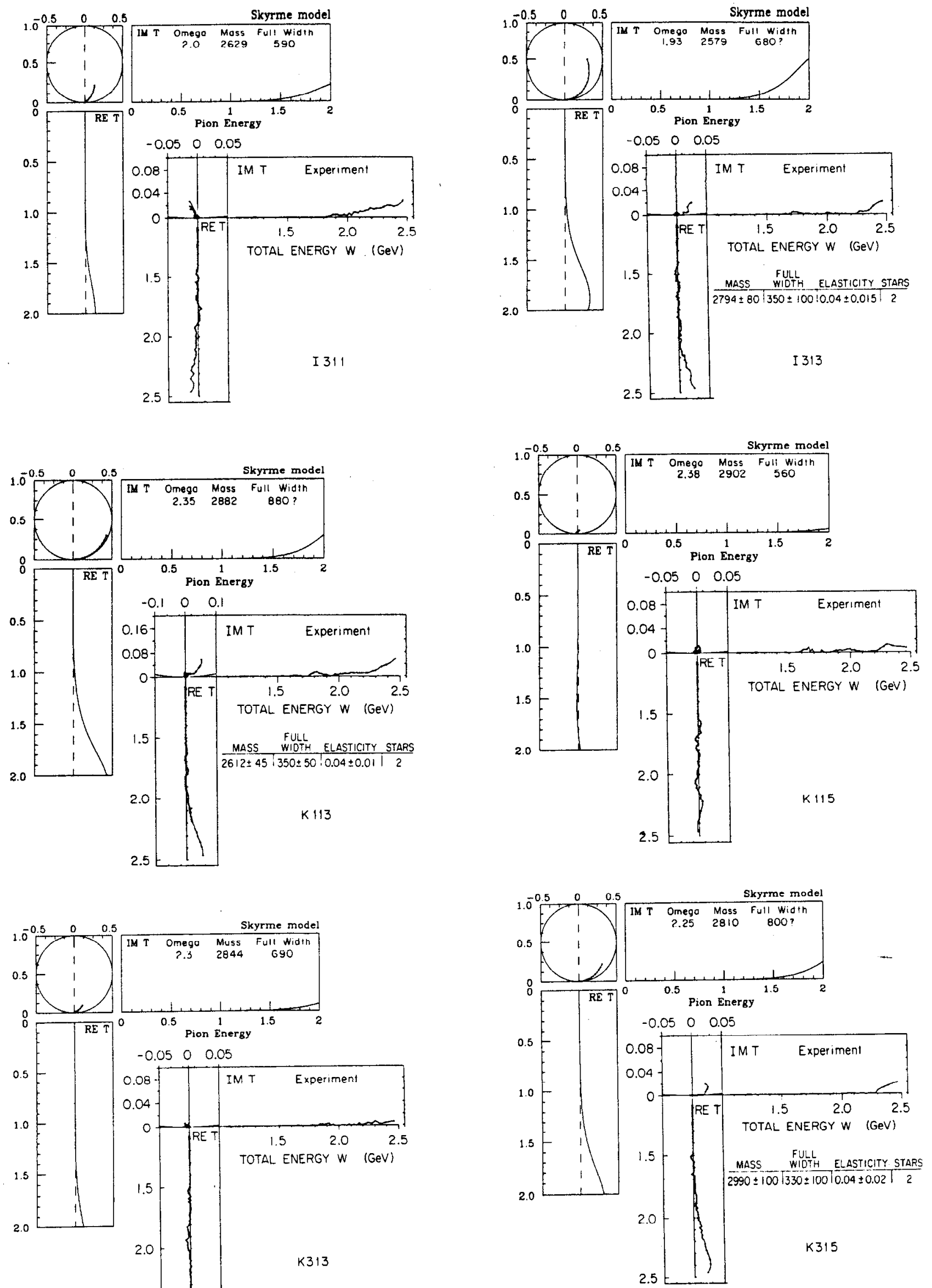

Fig. 19, cont. 
one that is extremely broad (and poorly defined) at $1.05 e f_{\pi}$. A similar sad story, albeit somewhat less egregious, is to be found in the $P_{11}$ channel; this is where the second-lightest resonance, the $N(1440)$, appears in Nature.

Interestingly, this was first noticed by Skyrme himself:

The $P$-wave meson-particle interaction [is] repulsive on the average. There is no indication of the strong attraction observed in the pion-nucleon resonant state, but this would hardly be expected in a static classical treatment where the rotational splitting of the particle states has been ignored. ${ }^{[6]}$

Yet these results are not necessarily fatal for the model. That is, despite the large discrepancies, one can argue that small perturbations in the $P$-wave sector of the theory can cause enormous effects in the corresponding Argand plots which could easily produce the observed real-world behavior for the amplitudes.

To see this, note that the physical $P$-wave amplitudes $\left(P_{11}\right.$ and $P_{33}$ especially) all contain contributions from the elementary $S$-matrix element $\mathbf{s}_{111}$, as is apparent from (III.21). This is the channel to which the rotational zero-mode of the skyrmion, Eq. (III.19a), couples at threshold. As a result, in the model, the $S$-matrix has a pole and a zero that have coalesced at the origin of the energy-plane for all four $P$-wave channels of $\pi N$ scattering. Now, one can easily envision effects which perturb these poles and zeroes away from the origin; certainly the quantization of the collective coordinates, which involves the next order in the $1 / N$ expansion, is one such effect. Consequently some of these poles might end up in the fourth quadrant, slightly below the positive real axis (Fig. 20a), while others might be pushed into the second quadrant (Fig. 20b). (These are quadrants of the 'second sheet.')

If this scenario actually takes place in the real world, what would we actually observe? The channels in which the poles have been perturbed into the fourth quadrant would contain clear $P$-wave resonances lying reasonably close to threshold: suggestively, the resonances our model lacks, the $\Delta(1232)$ and the $N(1440)$ are in fact the two lowest-lying excitations in pion-nucleon scattering. 

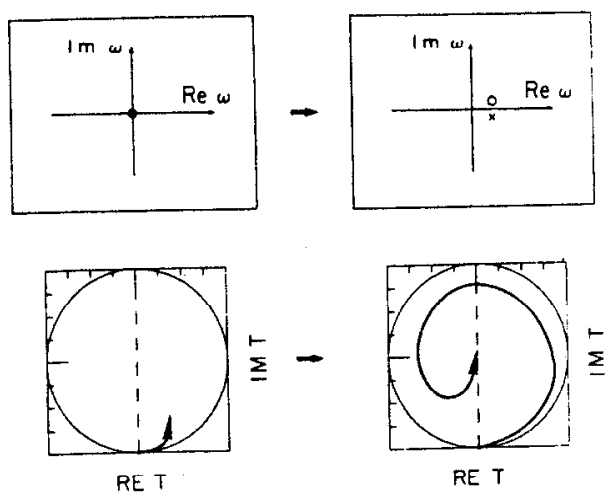

(a)
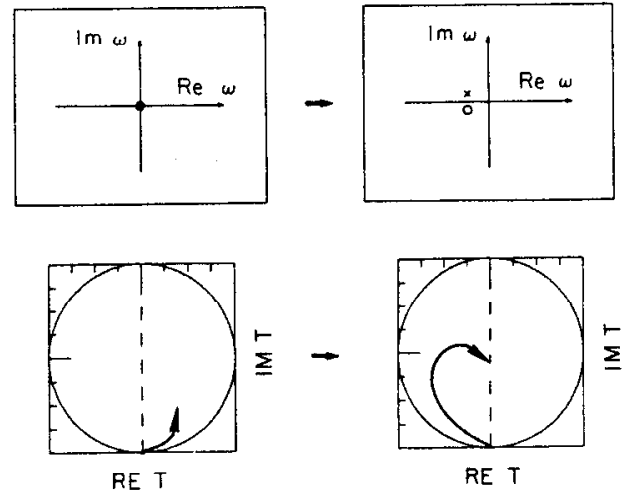

(b)
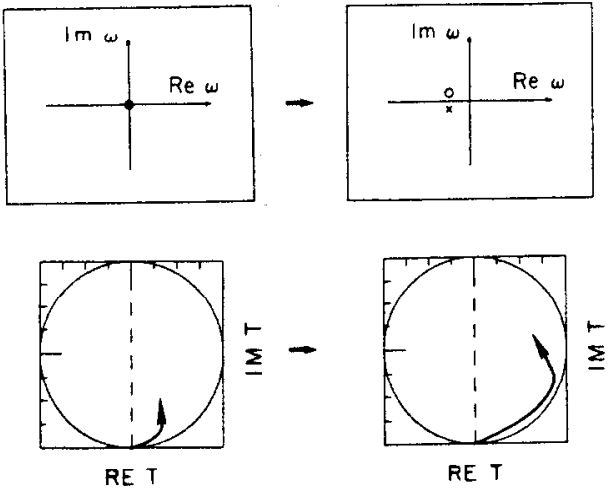

(c)

Fig. 20. Possible movement due to $1 / N$ corrections of the poles and zeroes of the $S$-matrix in the complex energy-plane, and the resulting effects on the amplitude near threshold. Poles are denoted by a cross and zeroes by a circle. 
In contrast, the channels in which the poles have been pushed into the second quadrant would be characterized by precisely the kind of repulsive behavior at low energies that one finds in the $P_{13}$ and $P_{31}$ amplitudes. Thus our scenario gives at least a consistent interpretation of the real-world $P$-wave amplitudes near threshold.

In a sense we already know that the delta-pole must be pushed in to the fourth quadrant by such higher-order corrections. This, after all, is the essence of the calculation in Ref. 13 leading to the mass formula (III.5). Recall that in the large$N$ expansion $f_{\pi} \sim N^{1 / 2}$ while $e \sim N^{-1 / 2}$. Consequently, according to Eq. (III.5) (which of course gets renormalized by additional $1 / N$ contributions), the nucleondelta mass-difference is proportional to $e^{3} f_{\pi}$ and hence of order $1 / N$, whereas typical excitation energies as obtained in the present analysis are measured in units of $e f_{\pi}$ which is of order unity. Thus it would actually have been inconsistent for the delta to appear in our lowest-order calculation. (Note that the ratio $\left(m_{\Delta}-m_{N}\right) / m_{N} \sim 1 / N^{2}$; this is just a special case of the well-known fact that the zero-modes of a soliton, when quantized, produce energy splittings of order $\hbar^{2}$, which in the large- $N$ approach is equivalent to $1 / N^{2}$.)

Before leaving the $P$-waves we ought to point out that the $P_{13}$ and $P_{31}$ amplitudes are already given quite nicely. Indeed, the standard lore is that the repulsive regions of Argand diagrams are very difficult to concoct in quark models of resonances, and so we consider it especially satisfactory to find such behavior emerging automatically from such a simple model. Nor is the agreement merely qualitative: the 'cusps' in the real-world $P_{13}$ and $P_{31}$ diagrams occur at 1530 and $1560 \mathrm{MeV}$, respectively, while the Skyrme-model prediction is $1640 \mathrm{MeV}$ in each case.

We turn next to the $S$-wave channels, where we find a similar discrepancy. In particular the model fails to reproduce the observed initial repulsive behavior of the amplitude in the $S_{31}$ channel. But the $S$-waves couple to the translational modes of the soliton, Eq. (III.19b). Thus just as for the $P$-waves one can argue 
that a small perturbation of the form depicted in Fig. 20b would induce such behavior. The situation for $S_{11}$ is not so clear: If one considers the real-world resonance at $1526 \mathrm{MeV}$ to be 'close' to threshold than presumably it is Fig. 20a that gives the correct picture; otherwise it is Fig. 20c.

In short, we have outlined a framework according to which all the $S$ - and $P$-wave amplitudes in the real world can be understood as arising from higherorder corrections in an underlying chiral-soliton model such as Skyrme's. In particular, repulsive behavior near threshold arises in this picture from $S$-matrix poles that have been perturbed from the origin into the first or second quadrant. (Reassuringly, the only amplitude other than $S_{31}, P_{31}$ and $P_{13}$ which exhibits such behavior in the real world is $D_{35}$, and this, too, mixes with the translational mode (III.19b).) Of course, at higher energies the effect of perturbing a threshold pole becomes negligible and so we would expect to see reasonable agreement once again between the model and experiment, as in fact we do in the $S$ - and $P$-waves.

We turn now to the higher waves, which fortunately present no such problems. We can be brief since the graphs, for better or for worse, speak for themselves. By way of a conclusion we offer the following observations:

1. The partial waves with $L \geq 2$ are on the whole in very satisfactory agreement with Nature. Many of the discrepancies in the higher waves can obviously be accounted for by the fact that our simple approach does not allow for the plethora of inelastic processes that occur in the real world; consequently our Argand plots stick too closely to the rim of the unitarity circle, and are simply much too large. Ideally one should allow for multiple pion production, other mesons and/or strangeness ( $c f$. Chapter IV).

2. The $F$-wave plots are in particularly close correspondence with experiment; this point has already been made in Ref. 18 . Note that these are the first channels which do not mix with the zero-modes of the skyrmion. In the $F_{35}$ channel a speed-analysis actually revealed two overlapping resonances in the model at 1831 
and $2032 \mathrm{MeV}$. Suggestively, the experimental data seems likewise characterized by a double peak, implying "that there might be additional structure, but the data do not allow additional structures to be resolved." ${ }^{[36]}$ Consequently the assignment in the real world is to a single broad ( $\Gamma=260 \pm 20 \mathrm{MeV}) F_{35}$ resonance at $1905 \mathrm{MeV}$. (Interestingly, a similar splitting of the $F_{35}$ resonance is predicted by the quark model. ${ }^{[26]}$

3. Even in those channels where the Argand diagrams are not reproduced very well, the model accurately predicts the locations of resonances with an appropriate choice of $e$ and $f_{\pi}$ (see Table I). In fact, almost all of the masses are given to within $16 \%$ of their actual values, and a majority are given to $6 \%$. This holds for all known resonances up to $3 \mathrm{GeV}$, which is surprising for a "lowenergy" theory. The general rule that masses increase with increasing partial wave comes out naturally, while the model correctly pinpoints several exceptions to this rule in the lower waves.

4. A serious discrepancy is that, except for $P_{33}$, the model fails to predict more than one resonance at reasonable energies in the channels where it should do so. In particular the model misses three 3- or 4-star resonances, namely the $S_{11}(1650), S_{31}(1900)$ and $D_{13}(1700)$, in addition of course to the delta and the Roper resonance as we discussed at length.

5. Except for the $F$ - and $G$-waves, the model predicts widths that are too large by roughly $50 \%$ or more. (Question-marks following some of our width assignments indicate a strong background phase-shift to the right of the resonance which makes a precise determination of the widths difficult.) Note that, unlike the quark model, there is no particular reason in the Skyrme model why resonances should be narrow.

6. Finally, the Skyrme model accurately mimics the big-small-small-big pattern discussed in Chapter II. This is because the dynamical assumption that 
$\mathbf{s}_{L+1, L L}$ varies negligibly from unity in comparison to $\mathbf{s}_{L-1, L L}$ and $\mathbf{s}_{L L L}$ is true for the Skyrme model, as we have seen.

Our results for the mass spectrum are presented in Table I. They are based on a least-squares fit with all resonances weighted equally. In fit \#1 we fixed the proton mass, leaving only one free parameter, while in fit \#2 we allowed the proton mass to vary. The optimal values for the Skyrme parameters turn out to be $\left\{e=6.29, f_{\pi}=142 \mathrm{MeV}\right\}$ and $\left\{e=4.79, f_{\pi}=150 \mathrm{MeV}\right\}$, respectively. An alternative approach would be to fix both the proton and the delta mass using (III.5), which gives ${ }^{[13]}\left\{e=5.45, f_{\pi}=129 \mathrm{MeV}\right\}$, but this yields a much poorer fit to the spectrum as a whole. (This is not too surprising since specifying the nucleon-delta mass-difference involves a fine-tuning to order $1 / N$.) In light of our earlier discussion we have chosen to compare our lowest-lying excitations in the $P_{11}$ and $P_{33}$ channels, not with the Roper and the delta, but with the nexthigher resonances in those channels; our 'predictions' in Table I for the deltamass merely come from Eq. (III.5). In all other cases where there was more than one resonance in a channel we compared the Argand plots to determine which resonance we should actually use.

Note that fit \#1 gives a nucleon-delta mass-difference that is much too large; in fact, it inverts the ordering of the first two $P_{33}$ resonances. Furthermore the corresponding Skyrme parameters yield substantially worse static properties of the model when plugged into the formulas obtained in Ref. 13. For these reasons we prefer fit \#2, which actually improves some of these properties, at the expense of allowing a proton mass of $1190 \mathrm{MeV}$ (from Eq. (III.5)); it is these mass assignments that we have noted in the Argand plots.

Table II lists a handful of static properties that were first calculated ${ }^{[13]}$ in the Skyrme model by Adkins, Nappi and Witten (ANW). The middle column lists their predictions for the proton and neutron magnetic moments, the axial coupling constant, and the mean isoscalar and isoscalar magnetic radii; the third column gives the same quantities recalculated using the values for the Skyrme 
parameters given by fit \#2; and the first column lists the experimental results. In summary we find it intriguing that this simple two-parameter model could yield a reasonable fit to such a wide range of both static and dynamic properties of hadrons. 
Table I

Comparison between experiment and Skyrme model predictions for baryon resonances

\begin{tabular}{|c|r|r|r|r|r|}
\hline Channel & Experiment & Fit \#1 & \% error & Fit \#2 & \% error \\
\hline S11 & 1526 & 1295 & -15 & 1478 & -3 \\
\hline S31 & 1610 & 1295 & -20 & 1478 & -8 \\
\hline P11 & 939 & 939 & 0 & 1190 & 27 \\
\hline P11 & 1723 & 1233 & -28 & 1427 & -17 \\
\hline P13 & 1710 & 1919 & 12 & 1982 & 16 \\
\hline P31 & 1888 & 1919 & 2 & 1982 & 5 \\
\hline P33 & 1232 & 1436 & 17 & 1424 & 16 \\
\hline P33 & 1522 & 1242 & -18 & 1435 & -6 \\
\hline P33 & 1868 & 1874 & 0.3 & 1946 & 4 \\
\hline D13 & 1519 & 1589 & 5 & 1715 & 13 \\
\hline D15 & 1679 & 1625 & -3 & 1744 & 4 \\
\hline D33 & 1680 & 1616 & -4 & 1737 & 3 \\
\hline D35 & 1901 & 1607 & -15 & 1730 & -9 \\
\hline F15 & 1684 & 1723 & 2 & 1823 & 8 \\
\hline F17 & 2005 & 1954 & -3 & 2011 & 0.3 \\
\hline F35 & 1905 & $1856^{a}$ & -3 & $1931^{6}$ & 1 \\
\hline F37 & 1913 & 1714 & -10 & 1816 & -5 \\
\hline G17 & 2140 & 2034 & -5 & 2075 & -3 \\
\hline G19 & 2268 & 2230 & -2 & 2234 & -2 \\
\hline G37 & 2215 & 2141 & -3 & 2162 & -2 \\
\hline G39 & 2468 & 2043 & -17 & 2083 & -16 \\
\hline H19 & 2205 & 2346 & 6 & 2327 & 6 \\
\hline H39 & 2217 & 2444 & 10 & 2407 & 9 \\
\hline H311 & 2416 & 2346 & -3 & 2327 & -4 \\
\hline I111 & 2577 & 2631 & 2 & 2558 & -1 \\
\hline I313 & 2794 & 2658 & -5 & 2579 & -8 \\
\hline K113 & 2612 & 3032 & 16 & 2882 & 10 \\
\hline K315 & 2990 & 2943 & -2 & 2810 & -6 \\
\hline & & & & & \\
\hline
\end{tabular}

a Average of two peaks at 1732 and $1981 \mathrm{MeV}$.

${ }^{b}$ Average of two peaks at 1831 and $2032 \mathrm{MeV}$.

Fit \# 1 - Nucleon mass fixed.

Fit \# 2 - Nucleon mass allowed to vary. 


\section{Table II}

Static properties in the Skyrme model

\begin{tabular}{|c|c|c|c|}
\hline Quantity & Experiment & ANW & Fit \#2 \\
\hline$f_{\pi}$ & $186 \mathrm{MeV}$ & $129 \mathrm{MeV}$ & $150 \mathrm{MeV}$ \\
\hline$e$ & $?$ & 5.45 & 4.79 \\
\hline$\mu_{p}$ & 2.79 & 1.87 & 2.23 \\
\hline$\mu_{n}$ & -1.91 & -1.31 & -1.81 \\
\hline$g_{A}$ & 1.23 & .61 & .79 \\
\hline$\left\langle r^{2}\right\rangle_{I=0}^{1 / 2}$ & $.72 \mathrm{fm}$ & $.59 \mathrm{fm}$ & $.58 \mathrm{fm}$ \\
\hline$\left\langle r^{2}\right\rangle_{M, I=0}^{1 / 2}$ & $.81 \mathrm{fm}$ & $.92 \mathrm{fm}$ & $.90 \mathrm{fm}$ \\
\hline
\end{tabular}




\section{EXTENSION TO THREE FLAVORS}

\section{Formalism for 3-Flavor Scattering}

It is important to consider the effect of incorporating additional low-lying mesons into our analysis. In this chapter we shall discuss how to extend the scattering formalism to the case of three light flavors, restricting ourselves to the idealized case of exact $S U(3)_{\text {favor }}$. We shall focus in particular on how the introduction of strangeness modifies the 2-flavor results of the last two chapters.

The unrotated skyrmion can be embedded in an $S U(2)$ subgroup of $S U(3)$ as follows: ${ }^{[8,27]}$

$$
U_{0}=\exp \left\{i F(r) \sum_{i=1}^{3} \hat{\mathbf{r}}^{i} \lambda^{i}\right\}
$$

with $\lambda^{a}, a=1, \ldots, 8$, the Gell-Mann matrices. ${ }^{\star}$ A novel feature of models with three or more flavors is that the effective Lagrangian must be augmented by the so-called Wess-Zumino term: ${ }^{\dagger}$

$$
-\frac{i N}{240 \pi^{2}} \int d^{5} x \epsilon^{i j k l m} \operatorname{Tr}\left(U^{\dagger} \partial_{i} U U^{\dagger} \partial_{j} U U^{\dagger} \partial_{k} U U^{\dagger} \partial_{l} U U^{\dagger} \partial_{m} U\right)
$$

which correctly reproduces the flavor-current anomalies of the strong interactions; here the integration is over the manifold $S^{3} \times D^{2}$ whose boundary is compactified space-time $S^{3} \times S^{1} .^{[8,28 \mid}$ Although, strictly speaking, the space of collective coordinates should be taken to be $S U(3) / U(1)_{\text {hypercharge }}{ }^{[27,8]}$ it turns out that for physically relevant baryon representations, one can allow the collective coordinates to range conveniently over the full $S U(3) .{ }^{[27,17]}$

* The alternative, "spin-1" embedding of the skyrmion turns out to have baryon number two.

$\dagger$ Our conventions are $\epsilon^{01234}=\epsilon^{0123}=-\epsilon_{0123}=-1$. 
Experience teaches us that it is fruitful to forget about the baryon's collective coordinates at first, and to concentrate instead on the simplified problem of a pseudoscalar-octet meson $\phi^{a}$ scattering from an unrotated skyrmion in its canonical hedgehog orientation (IV.1); we shall again refer to this as reduced scattering. Following the 2-flavor example, these mesons are naturally incorporated into the Lagrangian by letting

$$
U_{0}=\exp \left\{i F(r) \sum_{i=1}^{3} \hat{\mathbf{r}}^{i} \lambda^{i}\right\} \longrightarrow \exp \left\{i F(r) \sum_{i=1}^{3} \hat{\mathbf{r}}^{i} \lambda^{i}+\frac{2 i}{f_{\pi}} \sum_{a=1}^{8} \phi^{a} \lambda^{a}\right\}
$$

with $\lambda^{a}$ the Gell-Mann matrices. The Lagrangian is then expanded about the skyrmion to quadratic order in the $\phi$ 's. In particular, the WZ term makes a contribution

$$
\frac{i N}{4 \pi^{2}} \int d^{4} x \frac{F^{\prime} \sin ^{2} F}{F^{2} r^{2}}(1-\cos F)\left(K^{-} \dot{K}^{+}+\bar{K}^{0} \dot{K}^{0}\right)
$$

to the action. ${ }^{*}$ As always, cubic and higher terms are ignored in our lowest-order treatment, since they are suppressed by powers of $1 / f_{\pi} \sim 1 / \sqrt{N}$. The result is a set of linear Euler-Lagrange equations for the $\phi$ 's.

As in the 2-flavor procedure, we can obtain an effective radial problem by expanding $\phi^{a}$ in eigenstates of the symmetries of the unrotated skyrmion: in this case, $\left(\mathbf{K}^{2}, K_{z}, Y\right)$. Here $\mathbf{K}$ (not to be confused with the kaon!) is the vectorial sum $\mathbf{I}+\mathbf{L}$ of the meson's isospin and angular momentum, ${ }^{\ddagger}$ and $Y$ is its hypercharge (proportional to $\lambda^{8}$ ). Specifically, these fluctuations decompose into the following noninteracting sectors:

(a) There are the vector spherical harmonics familiar from the 2-flavor analysis, Eq. (III.8), where the first, second and third components stand for fluctuations in the $\pi^{+}, \pi^{0}$ and $\pi^{-}$directions, respectively. These are definite states of

* This contrasts with the baryon-number-zero sector of the theory, in which the WZ term first contributes to five-meson processes. ${ }^{[a, 8 \mid}$

$\ddagger$ We identify the isospin subgroup as the group generated by $\frac{1}{2} \lambda^{i}$ with $i=1,2,3$. 
$\mathbf{K}^{2}$ and $K_{z}$ by construction, and have $Y=0$. We will call the associated reduced $S$-matrices $\mathrm{s}_{K L^{\prime} L}^{\text {pion }}$ with $K=L-1, L, L+1$.

(b) Analogously, one can define "spinor spherical harmonics"

$$
y_{L}^{K K_{z}}(\Omega)=\left(\begin{array}{c}
\left\langle L \frac{1}{2}, K_{z}-\frac{1}{2}, \frac{1}{2} \mid K K_{z}\right\rangle Y_{L, K_{z}-\frac{1}{2}}(\Omega) \\
\left\langle L \frac{1}{2}, K_{z}+\frac{1}{2},-\frac{1}{2} \mid K K_{z}\right\rangle Y_{L, K_{z}+\frac{1}{2}}(\Omega)
\end{array}\right),
$$

where the first and second components stand for fluctuations in the $K^{+}$and $K^{0}$ directions, respectively. These have $Y=1$. By parity, $y_{K-\frac{1}{2}}^{K K_{F}}$ does not mix with $y_{K+\frac{1}{2}}^{K K_{z}}$, so that $L=L^{\prime}$. The associated reduced $S$-matrices will be called $\mathbf{s}_{K L}^{k a o n}$ with $K=L \pm \frac{1}{2}$. As is clear from (IV.4), the Wess-Zumino term contributes a term linear in time derivatives to the differential equations that determine the meson field (from which the phase-shifts are extracted). ${ }^{[20]}$

(c) Same as above, with the first and second component now standing for $-\bar{K}^{0}$ and $K^{-}$. These fluctuations have $Y=-1$. The corresponding reduced $S$-matrices, which we shall call $\mathrm{s}_{K L}^{k-b a r}$, are extracted from precisely the same differential equations as $\mathbf{s}_{K L}^{k a o n}$, except that the Wess-Zumino term contributes with the opposite sign. ${ }^{[20]}$ In the absence of the Wess-Zumino term, we would have $\mathbf{s}_{K L}^{k-b a r}(\omega) \equiv \mathbf{s}_{K L}^{k a o n}(\omega)$.

(d) Finally, fluctuations in the $\eta$ direction, expanded in the usual spherical harmonics. These have $\vec{K}=\vec{L}$ and $Y=0$. The corresponding reduced $S$-matrix is $\mathbf{s}_{L}^{\text {eta }}$.

This decomposition allows us to characterize the $S$-matrix for the process

$$
\phi^{a}(\vec{L})+\mathrm{URS} \rightarrow \phi^{b}\left(\vec{L}^{\prime}\right)+\mathrm{URS},
$$

with $\phi$ the meson field and URS standing for "unrotated skyrmion." Here $a$ and $b$ are $S U(3)$ flavor indices labeling the octet; each is short for the triplet of indices $\left(i, i_{z}, Y\right)$. When $a$ and $b$ are of the form $\left(1, i_{z}, 0\right)$, Eq. $(A .8)$ of Appendix A is 
valid. When $a$ and $b$ are both of the form $\left(\frac{1}{2}, i_{z},+1\right)$ or $\left(\frac{1}{2}, i_{z},-1\right)$, the relevant formula is

$$
\delta_{L L}, \sum_{K K_{*}}\left\langle K K_{z} \mid L \frac{1}{2} L_{z}, i_{z}\right\rangle\left\langle L^{\prime} \frac{1}{2} L_{z}^{\prime}, i_{z}^{\prime} \mid K K_{z}\right\rangle 8_{K L}
$$

where the reduced amplitude stands for $\mathbf{s}_{K L}^{k a o n}$ or $\mathbf{s}_{K L}^{k-b a r}$, respectively. And when $a=b=(0,0,0)$, the $S$-matrix is just

$$
\delta_{L L} \delta_{L_{*} L_{x}^{\prime}} \mathbf{8}_{L}^{\text {ta }}
$$

These formulas together form the 3-flavor analog of Eq. (A.8). The conserved quantities for the process (IV.6) are the sum $\mathbf{K}=\mathbf{I}+\mathbf{L}$ of the meson's isospin and angular momentum, as well as the meson's hypercharge $Y$. Of course, these are not preserved in physical 3-flavor meson-baryon scattering, for which the conserved quantities are the total meson-baryon angular momentum $\mathbf{J}$, and the total $S U(3)_{\text {flavor }}$ quantum numbers $\left\{R_{\text {tot }}, \gamma, I_{\text {tot }}, I_{z \text { tot }}, Y_{\text {tot }}\right\} .^{\star} \quad$ Pleasingly, we shall see momentarily that these conservation laws emerge naturally from the skyrmion formalism once the collective coordinate structure of the baryons is properly taken into account. Other physically relevant (albeit not necessarily conserved) quantum numbers are the meson partial wave $L$, and the spin $s$ and flavor representation $R$ of the baryon [i.e., $(s, R)=\left(\frac{1}{2}, \underline{8}\right)$ or $\left(\frac{3}{2}, \underline{10}\right)$ ].

In order to describe physical scattering, we need the analog of Eq. (II.8), with the integral now ranging over the group $S U(3)$. For this we require the correct $S U(3)$ generalization of the baryon wavefunctions, which is given by:

$$
\chi(A)=\frac{i}{\pi} \sqrt{\frac{\operatorname{dim} R}{2}}\left[D^{R}(A)^{-1}\right]_{a, b} \cdot(-1)^{s-s_{z}}
$$

where $a=\left(s,-s_{z}, 1\right), b=\left(i, i_{z}, Y\right)$, and $R$ denotes the representation of the

* Here $\gamma$ is a largely redundant index whose only real purpose is to distinguish between degenerate representations that can occur in the product of two $S U(3)$ representations, as for example the $8_{\mathrm{sym}}$ and $8_{\text {antisym }}$ in $8 \times 8$. It is not in general conserved, as is clear if one consider the non-vanishing coupling $\operatorname{Tr}(\{\bar{B}, \Phi\} \mid B, \Phi])$ of the baryon and meson octets. 
baryon. ${ }^{\dagger}$

The projection of the initial and final meson-baryon systems onto states of definite total $S U(3)$ quantum numbers is accomplished with the help of an $S U(3)$ Clebsch-Gordan coefficient

$$
\left\langle R_{1} i_{1} i_{z 1} Y_{1} ; R_{2} i_{2} i_{z 2} Y_{2} \mid R_{\text {tot }} \gamma I_{\text {tot }} I_{\mathrm{ztot}} Y_{\text {tot }}\right\rangle
$$

which can be factored conveniently into the product

$$
\left\langle i_{1} i_{2} i_{z 1} i_{z 2} \mid I_{\text {tot }} I_{\mathrm{ztot}}\right\rangle \cdot\left(\begin{array}{cc|c}
R_{1} & R_{2} & R_{\mathrm{tot}} \gamma \\
i_{1} Y_{1} & i_{2} Y_{2} & I_{\mathrm{tot}} Y_{\mathrm{tot}}
\end{array}\right)
$$

of an $S U(2)$ Clebsch with a so-called isoscalar factor. ${ }^{\text {[8ol }}$ The projection onto states of definite total angular momentum $\vec{J}$ is of course accomplished with ordinary $S U(2)$ Clebsches as before. As in the 2-flavor case, the physical $S$-matrix can then be expressed as a linear superposition of reduced amplitudes:

$\dagger$ These differ somewhat from the wavefunctions given in Ref. 27, which have nonstandard transformation properties under $I$ and $J$. The fact that the "left-handed hypercharge" is unity is a nontrivial quantization condition arising from consideration of the Wess-Zumino term. ${ }^{(27)}$ Our normalization in (IV.9) is such that $\int_{S U(9)} d A=2 \pi^{2}=\int_{S U(2)} d A$. 


$$
\begin{aligned}
& \mathbf{S}\left(\left[L s R R_{\text {tot }} \gamma I_{\text {tot }} I_{\text {stot }} Y_{\text {tot }} \vec{J}\right] \rightarrow\left[L^{\prime} s^{\prime} R^{\prime} R_{\text {tot }}^{\prime} \gamma^{\prime} I_{\text {tot }}^{\prime} I_{\text {stot }}^{\prime} Y_{\text {tot }}^{\prime} \vec{J}^{\prime}\right]\right) \\
& \delta_{R_{\text {tot }} R_{\text {tot }}^{\prime}} \delta_{I_{\text {tot }} I_{\text {tot }}^{\prime}} \delta_{I_{\text {stot }} I_{\text {stot }}^{\prime}} \delta_{Y_{\text {tot }} Y_{\text {tot }}^{\prime}} \delta_{J J^{\prime}} \delta_{J_{z} J_{x}^{\prime}} \quad \times \\
& \left\{\delta_{L L^{\prime}} \delta_{s s^{\prime}} \frac{\sqrt{\operatorname{dim} R \cdot \operatorname{dim} R^{\prime}}}{\operatorname{dim} R_{\mathrm{tot}}}\left(\begin{array}{c|cc}
R_{\mathrm{tot}} \gamma^{\prime} & R^{\prime} & 8 \\
s 1 & s 1 & 00
\end{array}\right)\left(\begin{array}{cc|c}
R & 8 & R_{\mathrm{tot}} \gamma \\
s 1 & 00 & s 1
\end{array}\right) \mathrm{s}_{L}^{\text {eta }}+\right. \\
& \sum_{I} \sqrt{\frac{\operatorname{dim} R \cdot \operatorname{dim} R^{\prime}}{(2 s+1)\left(2 s^{\prime}+1\right)}} \frac{2 I+1}{\operatorname{dim} R_{\mathrm{tot}}}\left[\delta_{L L^{\prime}}\left(\begin{array}{c|cc}
R_{\mathrm{tot}} \gamma^{\prime} & R^{\prime} & 8 \\
I 0 & s^{\prime} 1 & \frac{1}{2},-1
\end{array}\right) \times\right. \\
& \left(\begin{array}{cc|c}
R & 8 & R_{\text {tot }} \gamma \\
s 1 & \frac{1}{2},-1 & I 0
\end{array}\right) \sum_{K=L \pm \frac{1}{2}} \widetilde{P}_{L L s s^{\prime} I J K} \cdot \mathbf{s}_{K L}^{k-b a r}+ \\
& \delta_{L L^{\prime}}\left(\begin{array}{c|cc}
R_{\mathrm{tot}} \gamma^{\prime} & R^{\prime} & 8 \\
I 2 & s^{\prime} 1 & \frac{1}{2} 1
\end{array}\right)\left(\begin{array}{cc|c}
R & 8 & R_{\mathrm{tot}} \gamma \\
s 1 & \frac{1}{2} 1 & I 2
\end{array}\right) \sum_{K=L \pm \frac{1}{2}} \widetilde{P}_{L L s s^{\prime} I J K} \cdot \mathrm{s}_{K L}^{\text {kaon }}+ \\
& \left.\left.\left(\begin{array}{c|cc}
R_{\mathrm{tot}} \gamma^{\prime} & R^{\prime} & 8 \\
I 1 & s^{\prime} 1 & 1,0
\end{array}\right)\left(\begin{array}{cc|c}
R & 8 & R_{\mathrm{tot}} \gamma \\
s 1 & 1,0 & I 1
\end{array}\right) \sum_{K=L, L \pm 1} P_{L L^{\prime} s s^{\prime} I J K} \cdot \mathrm{s}_{K L^{\prime} L}^{\text {pion }}\right]\right\}
\end{aligned}
$$

This is the 3-flavor analog of Eq. (II.11). The $\tilde{P}$-symbols are defined exactly like the $P$-symbols, Eq. (II.10), with the single exception that the 1 's in the 6-j symbols are to be replaced by $\frac{1}{2}$ 's (reflecting the fact that kaons have isospin $\frac{1}{2}$ ). Only half-integral values of the index $I$ contribute to the coefficient of $\mathbf{s}^{\text {pion }}$, while only integral values contribute for $\mathrm{s}^{\text {kaon }}$ and $\mathrm{s}^{k-b a r}$. The long string of Kronecker $\delta$ 's in the first line of Eq. (IV.10) expresses the reassuring fact that total angular momentum and $S U(3)_{\text {flavor }}$ are conserved in the scattering process, as promised.

The explicit forms of the group-theoretic coefficients multiplying the reduced amplitudes in (IV.10) are presented in Appendix D. We have focused there on the physically relevant cases where the initial baryon is in the octet, and the final baryon is in either the octet or decuplet.

As in the 2-flavor case, Eq. (IV.10) points the way to two possible avenues of inquiry. On the one hand, one can calculate the reduced amplitudes in a specific 
model, such as the 3-flavor Skyrme model. On the other hand, one can extract from Eq. (IV.10) a host of model-independent linear relations between scattering amplitudes in different representations of $S U(3)$ and different channels of total angular momentum, albeit in the same partial wave. We will consider both these approaches in turn.

\section{The 3-Flavor Skyrme Model}

We now consider the specific case of the chirally-invariant 3-flavor Skyrme model, whose Lagrangian is given by the sum of Eqs. (I.17) and (IV.2), and whose underlying skyrmion solution is given by Eq. (IV.1). The scattering of pseudoscalar mesons in this model from the baryon octet/decuplet is governed by Eq. (IV.10), with the following dynamical input:

1) The reduced amplitudes $s_{K L^{\prime} L}^{\text {pion }}$ are precisely the same as in the 2-flavor case, as a moment's thought will confirm. (These were depicted in Figs. 13-18.)

2) The Lagrangian which governs fluctuations in the $\eta$ (i.e., $\lambda^{8}$ ) direction is just that of a free field in this model. Consequently, $s_{L}^{\text {eta }}(\omega) \equiv 1$.

3) Consider next the fluctuations $\psi_{K K_{s} L}(r) e^{i \omega t}, K=L \pm \frac{1}{2}$, in the kaon direction, summed against the spinor spherical harmonics defined in Eq. (IV.5). The effective radial equation of motion for these fluctuations turns out to be:*

* We have multiplied the equation directly obtained from a variation of the Lagrangian by $2 F /(1-\cos F)$ and introduced the dimensionless variable $\tilde{r}=e f_{\pi} r$. 


$$
\begin{aligned}
& \left(\tilde{r}^{2}+2 \sin ^{2} F\right) \frac{d^{2}}{d \tilde{r}^{2}}\left(\psi_{K K_{x} L} / F\right) \\
& +\left[2 \tilde{r}-4 F^{\prime} \sin F+F^{\prime}(1+\cos F)\left(\frac{\tilde{r}^{2}}{\sin F}+6 \sin F\right)\right] \frac{d}{d \tilde{r}}\left(\psi_{K K_{x} L} / F\right) \\
& +\left\{[ L ( L + 1 ) - K ( K + 1 ) - \frac { 5 } { 4 } ] \left[2+3 F^{\prime \prime} \sin F+\frac{5 \sin ^{2} F}{\tilde{r}^{2}}-\left(F^{\prime}\right)^{2}\right.\right. \\
& \left.-(1+\cos F)\left(1+\frac{4 \sin ^{2} F}{\tilde{r}^{2}}-2\left(F^{\prime}\right)^{2}\right)\right]-(L-1)(L+2)\left[1+\frac{\sin ^{2} F}{\tilde{r}^{2}}+\left(F^{\prime}\right)^{2}\right] \\
& +\frac{2 F}{1-\cos F}\left[\left(\frac{\tilde{r}^{2}}{4}+2 \sin ^{2} F\right) F^{\prime \prime}+\frac{\tilde{r}}{2} F^{\prime}+F^{\prime 2} \sin 2 F-\frac{\sin 2 F}{4}-\frac{\sin ^{2} F \sin 2 F}{\tilde{r}^{2}}\right] \\
& \left.+\omega^{2}\left(\tilde{r}^{2}+2 \sin ^{2} F+\tilde{r}^{2}\left(F^{\prime}\right)^{2}\right)-\frac{\omega N}{\pi^{2}} F^{\prime} \sin ^{2} F\right\} \cdot\left(\psi_{K K_{x} L} / F\right)=0 \cdot
\end{aligned}
$$

Here the bracketed expression in the next to last line is the defining equation for $F$, Eq. (III.4), which vanishes identically. The final term in (IV.11) represents the effect of the WZ term; note that it gives an attractive contribution to the phase-shifts. Numerically, however, its contribution turns out to be extremely small. The reduced $S$-matrix elements $s_{K L}^{k a o n}, K=L \pm \frac{1}{2}$, are extracted from (IV.11) by the usual phase-shift analysis.

4) Finally, there are the fluctuations in the antikaon direction, likewise expanded in spinor spherical harmonics. The corresponding reduced amplitudes $\mathbf{s}_{K L}^{k-b a r}$ are also extracted from (IV.11), except that the WZ term contributes with the opposite sign (as recently noted in Ref. 29), yielding a repulsive contribution to the phase-shifts. Numerically, we find $s_{K L}^{k a o n} \cong s_{K L}^{k-b a r}$.

As a check on Eq. (IV.11), we note the existence of the four independent zero-mode $(\omega=0)$ solutions, two of type (3) and two of type (4), corresponding to $L=1, K= \pm K_{z}=\frac{1}{2}$, and $\psi(r)=F(r)$. These are the zero-modes associated with perturbing the canonical embedding of the skyrmion depicted in (IV.1) by an infinitesimal unitary transformation of the form $\exp \left(i \epsilon \lambda^{a}\right), a=4,5,6,7$. As a 


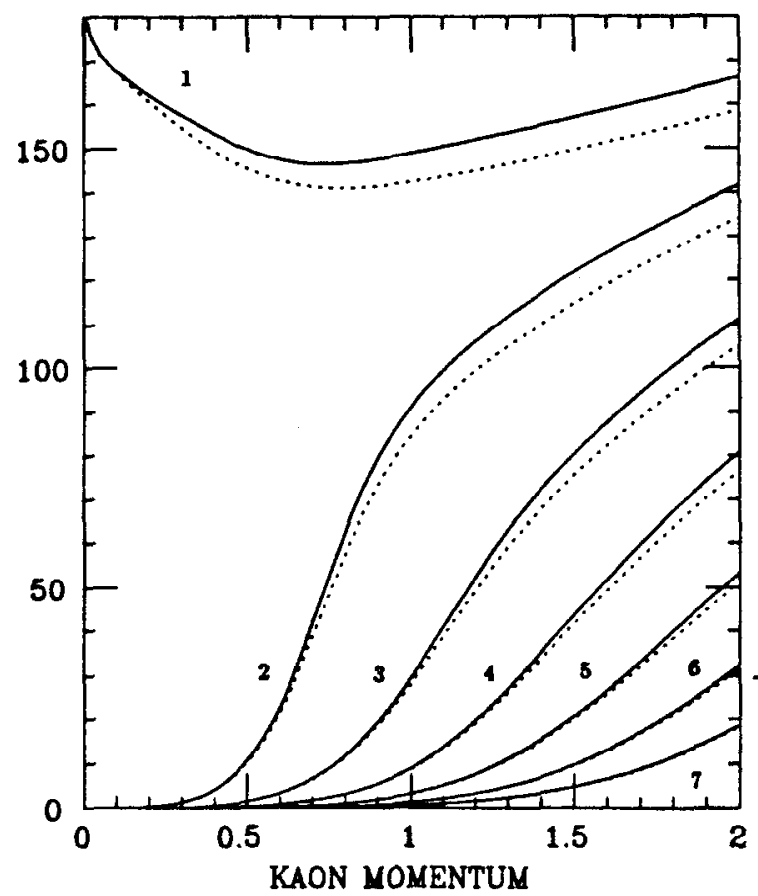

(a)

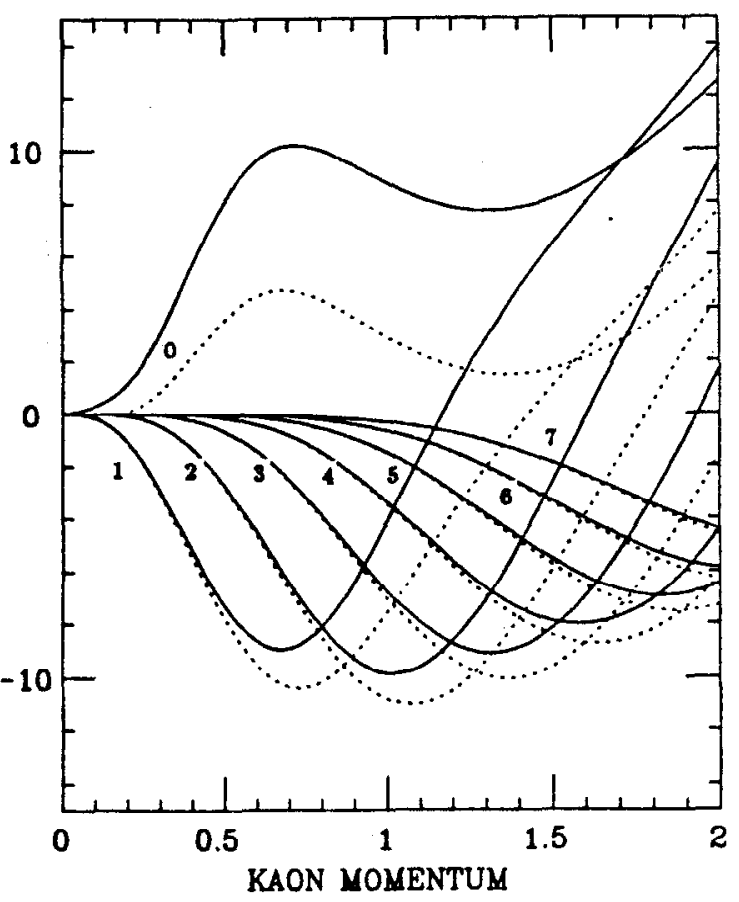

(b)

Fig. 21. Phase-shifts $\delta_{K L}^{k a o n}$ (solid lines) and $\delta_{K L}^{k-b a r}$ (dotted lines) plotted against kaon momentum in units of $e f_{\pi}$. Values of $L$ ranging from 0 to 7 are indicated. (a) The case $K=L-\frac{1}{2}$. (b) The case $K=L+\frac{1}{2}$.

second check on (IV.11), it is easy to verify that far away from the skyrmion, it correctly describes the propagation of a free particle in a partial wave $L$.

Figure 21 depicts the phase-shifts $\delta_{K L}^{k a o n}$ and $\delta_{K L}^{k-b a r}$ related to the reduced amplitudes via by $\mathrm{s}_{K L}^{\text {kaon }}=\exp \left(2 i \delta_{K L}^{\text {kaon }}\right)$, etc. It is clear that $\delta_{K L}^{\text {kaon }}(\omega) \approx \delta_{K L}^{k-b a r}(\omega)$, as asserted. Note that, for $L>1$, the phase-shifts with $K=L-\frac{1}{2}$ enjoy a 
much more dramatic rise than those with $K=L+\frac{1}{2}$; we shall make use of this observation later. In contrast, note the threshold repulsive behavior associated with $\delta_{\frac{1}{2} 1}^{k a a n}$ and $\delta_{\frac{1}{2} 1}^{k-b a r}$; this is the hallmark of the threshold boundstates in this channel mentioned above ( $c f$. Levinson's Theorem).

We are now in a position to compare 3-flavor meson-baryon scattering in the Skyrme model to Nature. We will focus first on the familiar process $\pi N \rightarrow \pi N$. Note that, according to Eq. (IV.10), there are contributions to this process from the "strange" reduced amplitudes $\mathbf{s}_{K L}^{k a 0 n}$ and $\mathbf{s}_{K L}^{k-b a r}$.

Figure 22 shows both the 2- and 3-flavor Skyrme model amplitudes for a couple of representative partial waves compared with experiment. Clearly the size of the amplitude has moved into somewhat closer agreement with Nature. The same pattern holds for most partial waves, and can be attributed to the opening-up of additional inelastic channels such as $\Sigma K$ in the 3-flavor approach. We should emphasize, however, that the poor agreement obtained in the previous chapter for the $P_{11}, P_{33}$ and $S_{31}$ channels is not improved; improvement in these channels must await a higher-order $1 / N$ analysis.

Figure 23 depicts the 2- and 3-flavor $H$-wave amplitudes in the Skyrme model. These illustrate the point that the 3-flavor Skyrme model does just as good a job as the 2-flavor model in mimicking the big-small-small-big pattern which characterizes the experimental curves for nearly all partial waves, as discussed in Chapter II. We will return to this phenomenon in the next section.

A particularly intriguing modification of the 2-flavor results occurs in the $F_{15}$ and $F_{37}$ channels (Fig. 24). The dominant peaks in these graphs indicate Skyrme-model resonances at roughly $1820 \mathrm{MeV}$, in reasonable accord with the 4star $F_{15}(1684)$ and $F_{37}(1913)$ states found in Nature. The interesting new feature is the emergence in the 3-flavor model of additional (weak) resonances at 2060 $\mathrm{MeV}$, in plausible correspondence with the observed 1-star $F_{15}(1882)$ and 2-star $F_{37}(2425)$. Suggestively, no such second peak emerges from the Skyrme model in the $F_{17}$ channel, where in Nature no second resonance is observed. The $F_{35}$ 

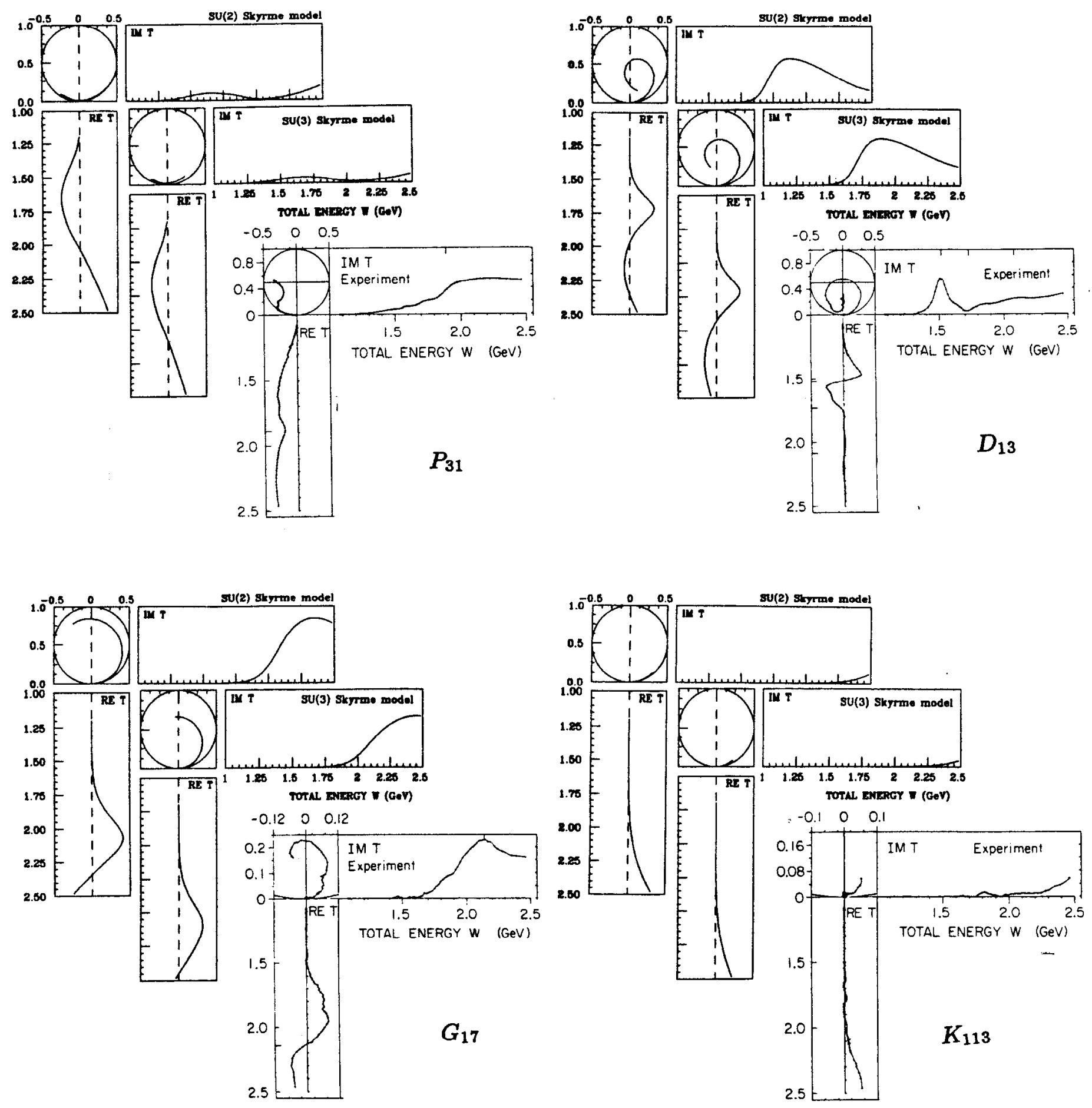

Fig. 22. Comparison of 2- and 3-flavor Skyrme model amplitudes with experiment for some representative partial waves 

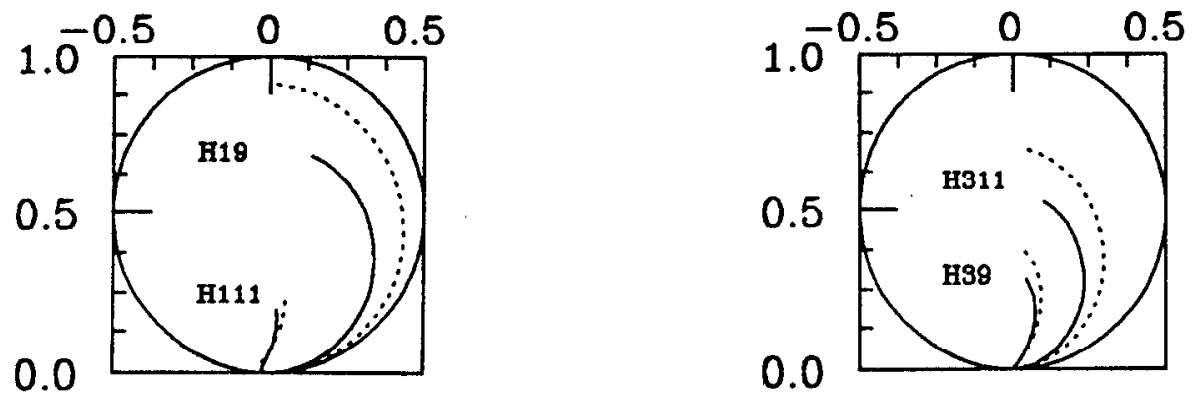

Fig. 23. The big-tmall-mmall-big pattern in both the 2-flavor (dotted lines) and 3-flavor (solid lines) Skyrme models.

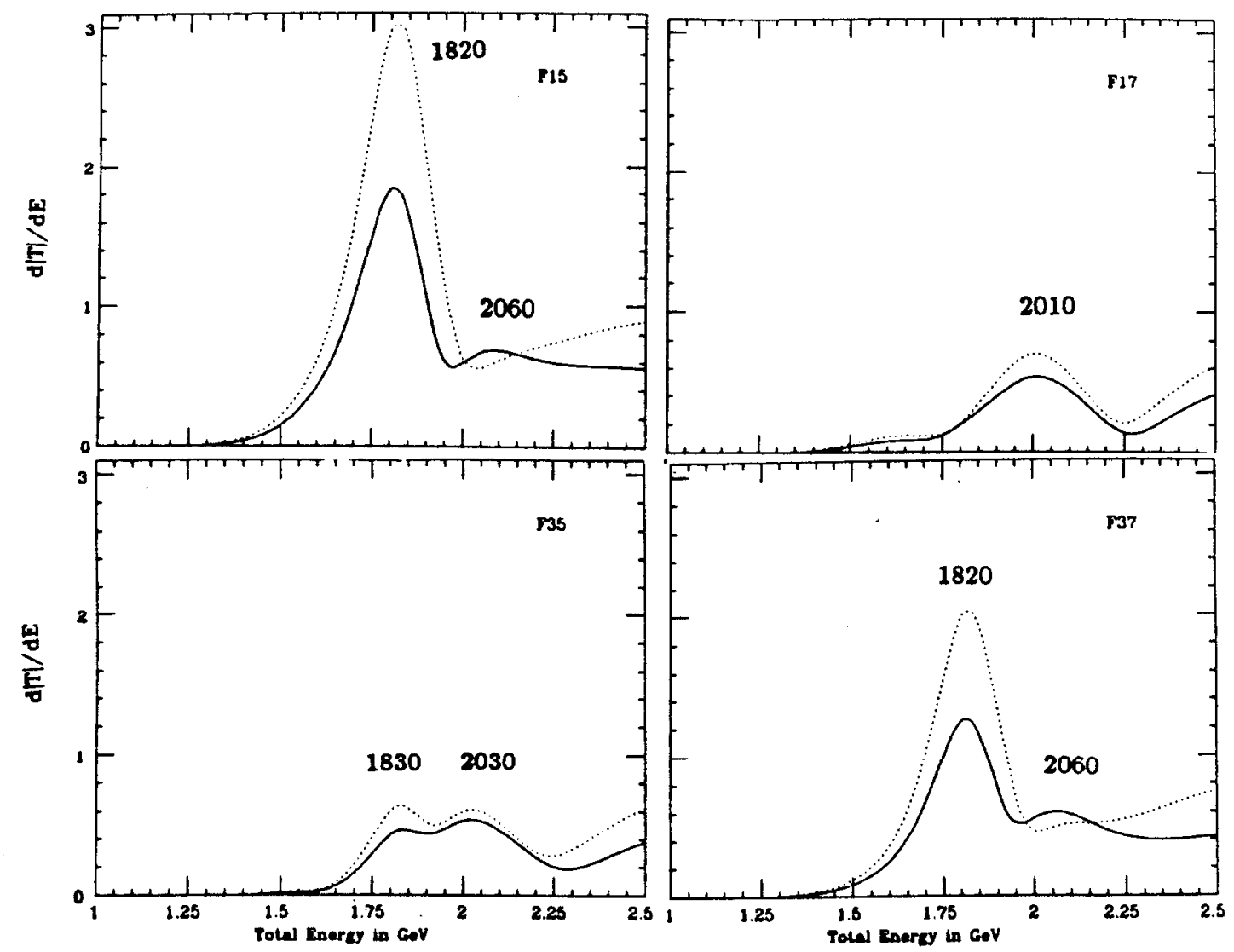

Fig. 24. Speed of $T$-matrix plotted against total c.o.m. energy in GoV for the four independent $F$-wave amplitudes in the 2-flavor (dotted lines) and 3-flavor (solid lines) Skyrme models. Resonance masses are indicated. Note the emergence of subsidiary resonances in $F_{15}$ and $F_{37}$ channels in the 3-flavor model. 
amplitude in both the 2- and 3-flavor models is characterized by two overlapping resonances at 1830 and $2030 \mathrm{MeV}$, although the experimental situation here is somewhat unclear, as discussed in the previous chapter (cf. Section III.2).

Finally, it should be emphasized that the values of the resonance masses are hardly affected by inclusion of strangeness, and the $8 \%$ "best-fit" agreement with experiment found in Chapter III continues to hold. Overall, the inclusion of a third light flavor improves the agreement between the Skyrme model and experiment for the 2-flavor process $\pi N \rightarrow \pi N$.

Of course, Eq. (IV.10) enables us to study $K N$ and $\bar{K} N$ scattering processes as well. However, these constitute much less rigid tests of the model because the experimental status of these processes is much more volatile than for $\pi N$. An additional problem for our purposes is that most of the experimental work has focused on $S$-, $P$ - and $D$-wave scattering, where, to leading order in $1 / N$, the Skyrme model can be expected to stumble due to mixing with zero-modes.

Figure 25 illustrates elastic $K N$ scattering in the Skyrme model versus experiment for a variety of partial waves. Although there is nothing particularly special about $K N$ scattering in the chiral soliton approach, it is very interesting from the point of view of the quark model, since resonances in these channels (unlike $\bar{K} N$ ) cannot correspond to $q q q$, but rather $q q q q \bar{q}$ states. The existence of such exotics has been the subject of considerable controversy over the past 15 years, with recent results tentatively favoring such states in the $P_{01}, P_{13}$ and $D_{03}$ channels. ${ }^{[31]}$ The Skyrme model results confirm the $D_{03}$ state. In addition, there appear to be very weak low-lying resonances in the $P_{01}$ and $P_{13}$ channels (note that there is actually counterclockwise activity in these curves before the cusps at $2 \mathrm{GeV}^{\star}$ ). In agreement with experiment, there are no low-lying Skyrme-model resonances in the $P_{11}$ and $P_{03}$ channels.

It is not clear that these results should be taken as anything more than suggestive, given the failure of the model to reproduce the observed repulsive

* This number assumes that excitation energies are measured from the $K N$ threshold. 

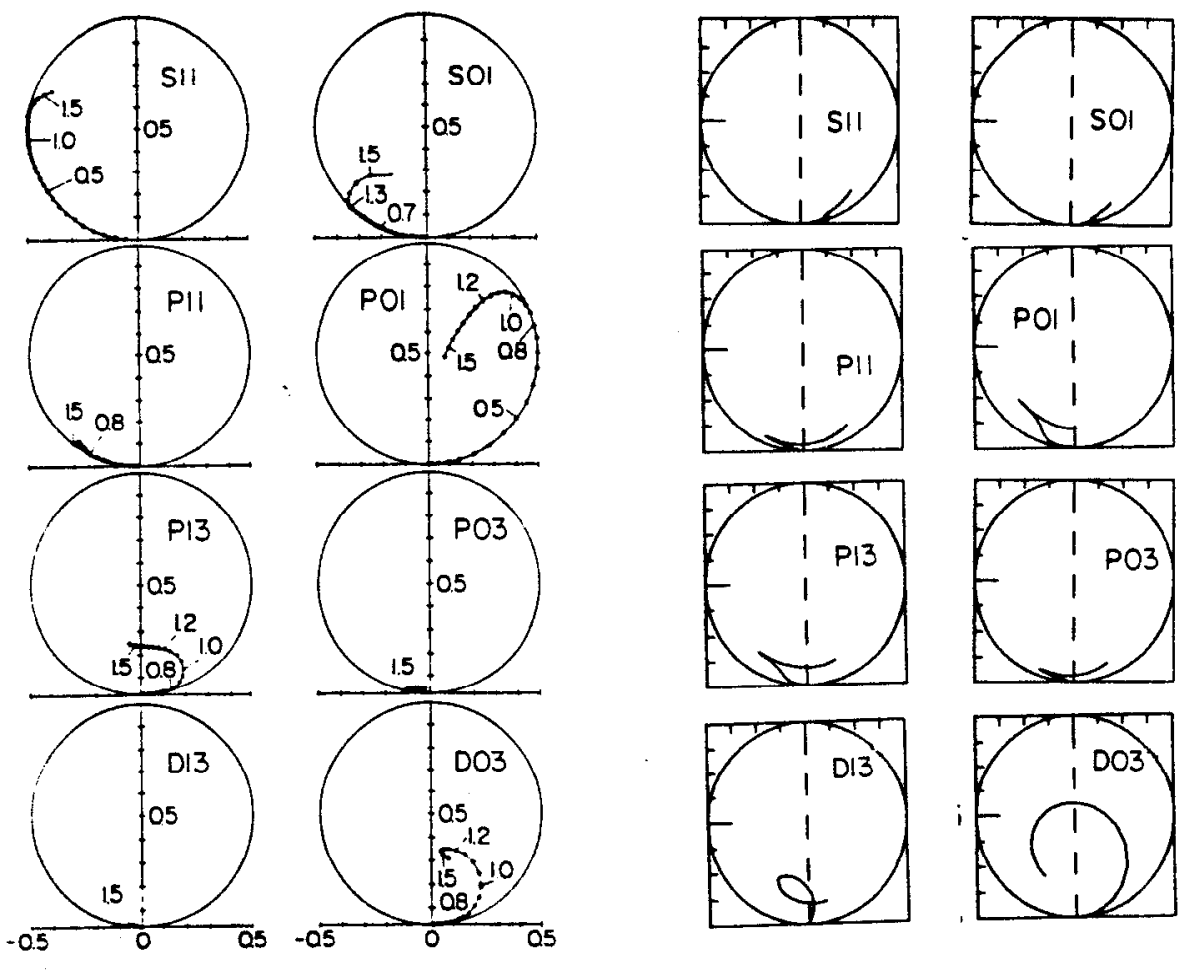

(a)

(b)

Fig. 25. Elastic $K N$ scattering amplitudes in a variety of partial waves. The notation is $L_{J, 2 J}$. (a) Experiment. $^{[81]}$ (b) The 3-flavor Skyrme model.

behavior in the $S$-waves. Furthermore, the Skyrme model predicts resonances in several channels where, in Nature, no counterclockwise behavior has been seen. Clearly a higher-order $1 / N$ analysis, in which the skyrmion's zero-modes have been properly disentangled, would be of great interest.

\section{Model-Independent Results}

We now leave the specific case of the 3-flavor Skyrme model behind, and apply Eq. (IV.10) directly to the study of real-world $\pi N$ amplitudes. Our operating assumptions are that a chiral soliton interpretation of the baryon is legitimate, and that the simultaneous approximations of large- $N$ and exact $S U(3)_{\text {favor }}$ (both of which entered crucially in the derivation of (IV.10)) are physically relevant.

A particularly notable success of the 2-flavor chiral soliton approach to $\pi N$ scattering was the natural emergence of the big-small-small-big pattern, as dis- 
cussed in Chapter II. The necessary dynamical input was the assumption that, for each value of $L$, the reduced amplitude $\mathbf{s}_{L+1, L L}$ varies negligibly from unity compared to the other two. A natural question is: Will the big-small-small-big pattern survive the extension to (unbroken) $S U(3)$ ?

It is clear from Table IV of Appendix D that the "big" physical amplitudes with $(I, J)=\left(\frac{1}{2}, L-\frac{1}{2}\right)$ or $\left(\frac{3}{2}, L+\frac{1}{2}\right)$ are built primarily out of the reduced amplitudes $\mathrm{s}_{L-1, L L}^{\text {pion }}, \mathrm{s}_{L L L}^{\text {pion }}, \mathrm{s}_{L-\frac{1}{2}, L}^{\text {kaon }}$, and $\mathrm{s}_{L-\frac{1}{2}, L}^{k-b a r}$. In contrast, the "small" amplitudes with $(I, J)=\left(\frac{1}{2}, L+\frac{1}{2}\right)$ or $\left(\frac{3}{2}, L-\frac{1}{2}\right)$ receive large contributions from $\mathrm{s}_{L+1, L L}^{\text {pion }}, \mathrm{s}_{L+\frac{1}{2}, L}^{\text {kaon }}$ and $\mathbf{s}_{L+\frac{1}{2}, L}^{\text {kaon }}$. Accordingly, if one makes the dynamical assumptions that, for each partial wave $L$, the deviations from unity of $\mathbf{s}_{L+1, L L}^{\text {pion }}, \mathbf{s}_{L}^{\text {eta }}, \mathbf{s}_{L+\frac{1}{2}, L}^{k a o n}$ and $\mathbf{s}_{L+\frac{1}{2}, L}^{k-b a r}$ are essentially negligible compared to the other four, then the pattern emerges automatically. (These assumptions are numerically the case in the $S U(3)$ Skyrme model, as we have just seen.) Furthermore, as in the 2-flavor case, the "big" amplitude with $I=\frac{1}{2}$ is predicted to be somewhat bigger than the amplitude with $I=\frac{3}{2}$, which holds experimentally for $L>1$ (cf. Fig. 9).

We next consider what model-independent linear relations follow from Eq. (IV.10). We will again focus our attention on the familiar 2-flavor processes $\pi N \rightarrow \pi N$ and $\pi N \rightarrow \pi \Delta$.

Let us represent the physical amplitudes for $\pi N \rightarrow \pi N$ by $\mathbf{S}_{L I J}^{\pi N N}$, with $L$ denoting the pion partial wave and $I$ and $J$ the total isospin and angular momentum of the pion-nucleon system. Eq. (IV.10) can be shown to imply:

$$
\begin{aligned}
& (4 L+2) \mathbf{S}_{L \frac{3}{2}, L-\frac{1}{2}}^{\pi \pi N}-(L-1) \mathbf{S}_{L \frac{1}{2}, L-\frac{1}{2}}^{\pi N N}-(3 L+3) \mathbf{S}_{L \frac{1}{2}, L+\frac{1}{2}}^{\pi N \pi} \\
& =\frac{13 L-5}{90}\left(\mathrm{~s}_{L-\frac{1}{2}, L}^{k a a n}-\mathbf{s}_{L-\frac{1}{2}, L}^{k-b a r}\right)+\frac{23(L+1)}{90}\left(\mathrm{~s}_{L+\frac{1}{2}, L}^{k a a n}-\mathrm{s}_{L+\frac{1}{2}, L}^{k-b a r}\right)
\end{aligned}
$$

and 


$$
\begin{aligned}
& (4 L+2) S_{L \frac{3}{2}, L+\frac{1}{2}}^{\pi N+N}-3 L S_{L \frac{1}{2}, L-\frac{1}{2}}^{\pi N+N}-(L+2) S_{L \frac{1}{2}, L+\frac{1}{2}}^{\pi N \pi N} \\
& =\frac{23 L}{90}\left(8_{L-\frac{1}{2}, L}^{k a a n}-8_{L-\frac{1}{2}, L}^{k-b a r}\right)+\frac{13 L+18}{90}\left(8_{L+\frac{1}{2}, L}^{k a a n}-\mathbf{s}_{L+\frac{1}{2}, L}^{k-b a r}\right)
\end{aligned}
$$

These equations relate the experimental $\pi N$ amplitudes to reduced amplitudes obtainable, in principle, from a phase-shift analysis of the "optimal" nonlinear sigma model of Nature. Unfortunately, this optimal nonlinear sigma model, obtainable in principle from $\mathrm{QCD}$, is unknown. Thus, without some further approximation, Eq. (IV.12) is entirely without predictive power.

However, using the 3-flavor Skyrme model as a guide (cf. Fig. 21), one can expect the right-hand sides of (IV.12) to be extremely small (note that they would vanish identically were it not for the $\mathrm{WZ}$ term). Accordingly, let us examine the linear relations between experimental amplitudes that result from setting the right-hand sides of (IV.12) to zero. Here we find a surprise: these are precisely the relations (II.18) which we derived, without any such dynamical assumption, from the 2-flavor formalism! Since, in general, these relations work quite well, the logical conclusion is that the $\mathrm{WZ}$ term probably makes only a very small numerical contribution to the real-world meson-baryon $S$-matrix. ${ }^{\dagger}$

It should be emphasized that we had no right to expect any relations, approximate or not, between physical $\pi N \rightarrow \pi N$ amplitudes to emerge from the 3-flavor formalism. The reason is the following. In the 2-flavor approach, the four physical $\pi N$ amplitudes for each $L$ (i.e., $J=L \pm \frac{1}{2}$ and $I=\frac{1}{2}, \frac{3}{2}$ ) are expressed through Eq. (II.11) as superpositions of only three reduced amplitudes. Consequently, at least one nontrivial relation between physical amplitudes is guaranteed (in fact, there are two). In contrast, in the 3-flavor approach, these same four amplitudes are linear combinations of eight reduced amplitudes. That the relations

* Recall that the WZ term vanishes in the 2 -flavor case.

$\dagger$ This conclusion differs from that of Ref. 29. 
turn out to be the same in both formalisms is cause for further surprise, since the group-theoretic factors multiplying the $\mathbf{s}_{K L L}^{\text {pion }} \mathrm{s}$ are completely different in the two approaches. (This can be seen by comparing Eq. (B.1) of Appendix B to Table IV in Appendix D.)

We can also extract from (IV.10) information about $\pi N \rightarrow \pi \Delta$. Let us represent the physical amplitudes for this process by $S_{L L^{\prime}}^{\pi * \Delta}$ w with $L^{\prime}$ the exiting pion partial wave (which can differ from $L$ by two). For the case $L=L^{\prime}$, Eq. (IV.10) implies:

$$
\begin{aligned}
& \mathrm{S}_{L L \frac{3}{2}, L-\frac{1}{2}}^{\pi N \pi \Delta}-\frac{4(L-1)}{\sqrt{10(2 L+1)} \cdot \mathrm{S}_{L L \frac{1}{2}, L-\frac{1}{2}}^{N \pi \Delta}} \\
& -\frac{3}{2 L+1} \sqrt{\frac{(L+1)(2 L+3)(2 L-1)}{10 L}} \cdot \mathbf{S}_{L L \frac{1}{2}, L+\frac{1}{2}}^{N \pi \Delta}= \\
& \frac{\sqrt{(2 L-1)(L+1)}}{18(2 L+1)}\left(8_{L-\frac{1}{2}, L}^{k a o n}-8_{L-\frac{1}{2}, L}^{k-b a r}-8_{L+\frac{1}{2}, L}^{k a o n}+8_{L+\frac{1}{2}, L}^{k-b a r}\right)
\end{aligned}
$$

and likewise

$$
\begin{aligned}
& \mathrm{S}_{L L \frac{3}{2}, L+\frac{1}{2}}^{\pi \pi \Delta}-\frac{3}{2 L+1} \sqrt{\frac{L(2 L+3)(2 L-1)}{10(L+1)}} \cdot \mathrm{S}_{L L \frac{1}{2}, L-\frac{1}{2}}^{\pi \pi \Delta} \\
& -\frac{4(L+2)}{\sqrt{10}(2 L+1)} \cdot \mathrm{S}_{L L \frac{1}{2}, L+\frac{1}{2}}^{\pi N \Delta}= \\
& \frac{1}{18(2 L+1)} \sqrt{\frac{(2 L+3)(2 L+1) L}{L+1}}\left(\mathrm{~s}_{L-\frac{1}{2}, L}^{k a a n}-\mathrm{s}_{L-\frac{1}{2}, L}^{k-b a r}-\mathrm{s}_{L+\frac{1}{2}, L}^{k a n}+\mathrm{s}_{L+\frac{1}{2}, L}^{k-b a r}\right)
\end{aligned}
$$

Setting the right-hand sides to zero as before, we again recover precisely the 2-flavor predictions (II.19). Similarly, for the case $L=L^{\prime} \pm 2$, Eq. (IV.10) implies the simple proportionality relations given by Eq. (II.20), with no "WZ corrections." (This is due to the $\delta_{L L^{\prime}}$ multiplying the kaon-like and antikaon-like reduced amplitudes in (IV.10).) 
In the 2-flavor case, there was for each $L$ one further model-independent prediction relating the processes $\pi N \rightarrow \pi N$ to $\pi N \rightarrow \pi \Delta$, namely Eq. (II.21), but this is lost in the 3-flavor formalism.

Thus, with the dynamical assumption $\mathbf{s}_{K L}^{k a o n} \approx \mathbf{s}_{K L}^{k-b a r}$ suggested by the 3-flavor Skyrme model, the 3-flavor formalism yields almost all the model-independent linear relations between experimental $\pi N$ scattering amplitudes that emerged from the 2-flavor approach. It is natural to explore the consequences of making additional dynamical assumptions about the optimal 2- and 3-flavor nonlinear sigma models of Nature.

A natural set of such assumptions is suggested by the big-small-small-big pattern. This pattern characterizes the broad class of 2-flavor models (Skyrme's included) for which the reduced amplitude $s_{L+1, L L}^{\text {pion }}$ vary neglibly from unity in comparison with $\mathbf{s}_{L-1, L L}^{\text {pion }}$ and $\mathbf{s}_{L L L}^{\text {pion }}$. Similarly, it emerges from 3-flavor models if, out of the eight reduced amplitudes for each partial wave, the variation of $\mathbf{s}_{L+1, L L}^{\text {pion }}, \mathbf{s}_{L}^{\text {eta }}, \mathbf{s}_{L+\frac{1}{2}, L}^{k a a n}$, and $\mathbf{s}_{L+\frac{1}{2}, L}^{k-b a r}$ is small compared with the others. Fortunately, we have the means of directly testing whether these dynamical assumptions are valid approximations as regards the "optimal" 2- and 3-flavor nonlinear sigma models of Nature. For, with these additional approximations, Eqs. (II.11) and (IV.10) can be shown to imply the extra relations:

$$
\begin{gathered}
9 L\left(\mathrm{~S}_{L \frac{1}{2}, L-\frac{1}{2}}^{\pi N \pi N}-1\right)+(3 L+6)\left(\mathrm{S}_{L \frac{1}{2}, L+\frac{1}{2}}^{\pi N N}-1\right) \\
\cong 9 L \sqrt{\frac{2 L-1}{L+1}} S_{L L \frac{1}{2}, L-\frac{1}{2}}^{\pi N \pi \Delta}+3(10 L+11) \sqrt{\frac{L}{2 L+3}} S_{L L \frac{1}{2}, L+\frac{1}{2}}^{\pi N \pi \Delta} \cdot
\end{gathered}
$$

and

$$
9 L\left(\mathrm{~S}_{L \frac{1}{2}, L-\frac{1}{2}}^{\pi \pi N}-1\right)+(3 L+6)\left(\mathrm{S}_{L \frac{1}{2}, L+\frac{1}{2}}^{\pi N * N}-1\right)
$$




$$
\cong 32 L \sqrt{\frac{2 L-1}{10(L+1)}} \mathbf{S}_{L L \frac{1}{2}, L-\frac{1}{2}}^{\pi N \Delta}+8(14 L+15) \sqrt{\frac{L}{10(2 L+3)}} \mathbf{S}_{L L \frac{1}{2}, L+\frac{1}{3}}^{\pi N \Delta},
$$

respectively.

Figure 26 displays these relations as applied to the experimental $\pi N \rightarrow \pi N$ and $\pi N \rightarrow \pi \Delta P-, D$ - and $F$-wave amplitudes. ${ }^{\star}$ Clearly, there is no substantial difference in agreement between the 2- and 3-flavor predictions. Although the overall magnitudes of the curves are not in particularly close accord, the shapes are roughly similar. Furthermore, the agreement in the signs of the amplitudes is in itself a completely nontrivial result (as a glance at the Argand plots of Ref. 14 will confirm). Certainly (IV.14) and (IV.15) are as successful as Eqs. (II.19)(II.21), which were derived without additional dynamical assumptions. In other words, incorporating these extra approximations does not noticeably worsen the agreement.

Our conclusion, suggested by the big-small-small-big pattern and reinforced by Fig. 26, is that the dynamical assumptions stated above are accurate descriptions of the 2- and 3-flavor effective Lagrangians derived from QCD. We hope that, as such, they will prove to be useful constraints on the current modelbuilding efforts in skyrmion physics.

* In order to make use of the available curves for $\pi N \rightarrow \pi \Delta,{ }^{[14]}$ we have combined Eqs. (IV.14) and (IV.15) with Eq. (II.19) in Figs. 26a and 26c. 


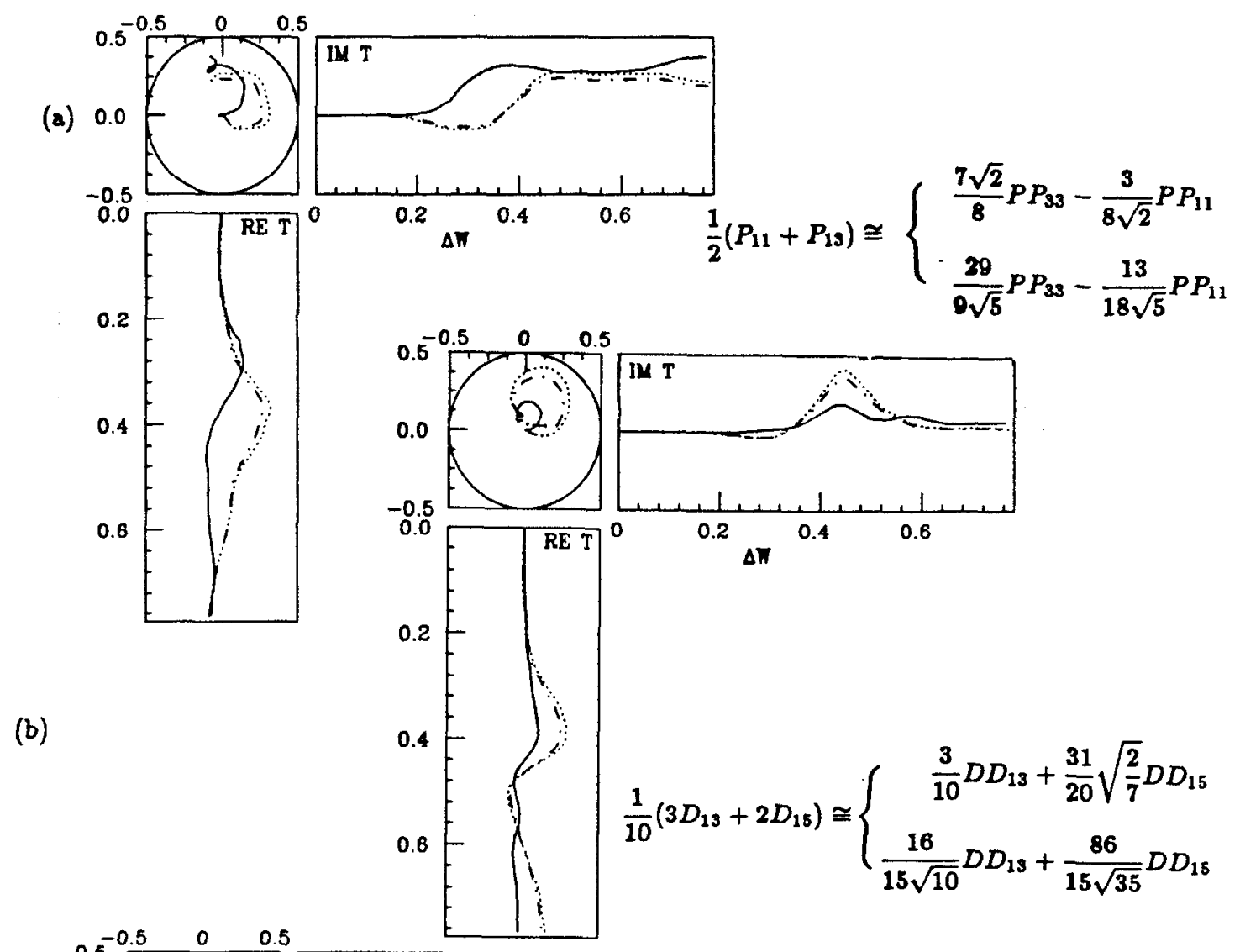

(c)

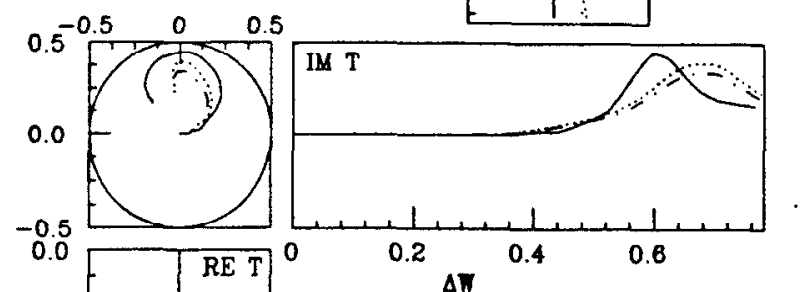

$$
\frac{1}{14}\left(9 F_{15}+5 F_{17}\right) \cong\left\{\begin{array}{l}
\frac{27}{14 \sqrt{2}} F F_{35}+\frac{11}{42} \sqrt{\frac{6}{5}} F F_{37} \\
\frac{26}{7 \sqrt{5}} F F_{35}+\frac{16 \sqrt{3}}{105} F F_{37}
\end{array}\right.
$$

Fig. 26. Test of Eqs. (IV.14) and (IV.15). The upper and lower expressions in braces are the 2- and 3-flavor predictions, depicted by dot-dash and dotted. lines, respectively. The expressions to the left of the equalities are depicted by solid lines. 


\section{APPENDIX A: Derivation of Equation (II.9)}

The purpose of this appendix is to fill in the steps between Eqs. (II.8) and (II.9). We shall not assume isospin and angular momentum conservation $a b$ initio; these will emerge in the course of our derivation.

We begin by considering the integral over the $S U(2)$ group manifold in (II.8). Changing variables to $A \epsilon^{-1}$ transforms the integral to

$$
\begin{aligned}
\int d A & D^{R^{\prime}}(A)_{i_{z}^{\prime} s_{z}^{\prime}}\left(D^{1}(A) \epsilon^{1}\right)_{i m}\left(\epsilon^{1} D^{1}(A)^{-1}\right)_{n j}\left(D^{R}(A)^{-1}\right)_{s_{x} i_{x}} \\
= & (-1)^{n-m} \int d A D^{R^{\prime}}(A)_{i_{z}^{\prime} s_{1}^{\prime}} D^{1}(A)_{i,-m}\left(D^{1}(A)^{-1}\right)_{-n, j}\left(D^{R}(A)^{-1}\right)_{s_{x} i_{x}}
\end{aligned}
$$

using

$$
\epsilon^{1}=\left(\begin{array}{ccc}
0 & 0 & 1 \\
0 & -1 & 0 \\
1 & 0 & 0
\end{array}\right)
$$

It turns out that the $A$-integration can be carried out explicitly, thanks to the Clebsch-Gordon decomposition

$$
D^{R_{1}}(A)_{a b} D^{R_{2}}(A)_{c d}=\sum_{\widetilde{R}} D^{\widetilde{R}}(A)_{a+c, b+d}\left\langle R_{1} R_{2} a c \mid \widetilde{R}, a+c\right\rangle\left\langle\widetilde{R}, b+d \mid R_{1} R_{2} b d\right\rangle
$$

and the orthogonality relation

$$
\int d A D^{R_{1}}(A)_{a b}\left(D^{R_{2}}(A)^{-1}\right)_{c d}=\frac{2 \pi^{2}}{2 R_{1}+1} \delta_{R_{1} R_{2}} \delta_{b c} \delta_{a d} .
$$

We obtain:

$$
\begin{aligned}
\left\langle\pi^{i} \pi^{j}\right\rangle_{R i_{z} s_{z} ; R^{\prime} i z_{z}^{\prime} s_{z}^{\prime}}= & \sum_{m n \tilde{R}}\left\langle\pi^{m} \pi^{n}\right\rangle_{\circ}(-1)^{n-m} \frac{\sqrt{(2 R+1)\left(2 R^{\prime}+1\right)}}{2 I+1} \\
& \times\left\langle R^{\prime} 1 i_{z}^{\prime} i \mid \tilde{R}, j+i_{z}\right\rangle\left\langle\tilde{R}, s_{z}-n \mid R^{\prime} 1 s_{z}^{\prime},-m\right\rangle \\
& \times\left\langle R 1 s_{z},-n \mid \tilde{R}, s_{z}-n\right\rangle\left\langle\tilde{R}, j+i_{z} \mid R 1 i_{z} j\right\rangle
\end{aligned}
$$


It is useful to project the initial and final pion-baryon states onto states of definite total isospin $\left|I I_{z}\right\rangle$ and $\left|I^{\prime} I_{z}^{\prime}\right\rangle$, respectively. A straightforward calculation yields the somewhat simpler expression

$$
\begin{aligned}
\langle\pi \pi\rangle_{R \vec{I}_{x} ; R^{\prime} \vec{I}^{\prime} s_{z}^{\prime}}= & \delta_{I I^{\prime}} \delta_{I_{z} I} \frac{\sqrt{(2 R+1)\left(2 R^{\prime}+1\right)}}{2 I+1} \\
& \times \sum_{m n}(-1)^{n-m}\left\langle\pi^{m} \pi^{n}\right\rangle_{0} \\
& \times\left\langle I, s_{z}-n \mid R^{\prime} 1 s_{z}^{\prime},-m\right\rangle\left\langle R 1 s_{z},-n \mid I, s_{z}-n\right\rangle .
\end{aligned}
$$

Happily, isospin conservation is now manifest in the Kronecker-deltas.

We have not yet arrived at our final destination, where the initial and final pion-baryon states are characterized, not only by definite isospin, but by definite angular momentum as well. But in order to make progress we must necessarily return to the question of diagonalizing the differential operators $\widehat{\mathbf{L}}_{i j}$ defined in Eqs. (II.3) and (II.4). Complicated though $\hat{\mathbf{L}}$ may be, it respects the symmetry $\vec{K} \equiv \vec{I}($ pion $)+\vec{L}($ pion $)$. Consequently $\hat{\mathbf{L}}$ preserves the subspaces of states of definite $K$ and $K_{z}$.

How do we construct these subspaces? Consider the quantity

$$
\Pi_{L}^{K K_{z}}(\Omega)=\left(\begin{array}{c}
\left\langle L 1 K_{z}-1,1 \mid K K_{z}\right\rangle Y_{L, K_{z}-1}(\Omega) \\
\left\langle L 1 K_{z} 0 \mid K K_{z}\right\rangle Y_{L K_{z}}(\Omega) \\
\left\langle L 1 K_{z}+1,-1 \mid K K_{z}\right\rangle Y_{L, K_{z}+1}(\Omega)
\end{array}\right)
$$

these are the vector spherical harmonics. By the familiar rules for addition of angular momenta $\Pi_{L}^{K K_{z}}$ is indeed a state of definite $K$ and $K_{z}$. Unfortunately it also has definite orbital angular momentum $L$, which is not preserved by $\hat{\mathbf{L}}$ : the pion can jump two units in $L$ in the process $\pi N \rightarrow \pi \Delta$, for example, in a manner consistent with angular momentum conservation. Therefore, in order to block-diagonalize $\hat{\mathbf{L}}$ we must sum over all allowed values of $L$ for each $K$, namely 
$L=(K-1, K, K+1)$. Parity precludes the states with $L=K$ from mixing with those with $L=K \pm 1$; consequently the eigenstates of $\widehat{\mathbf{L}}$ are of the following form:

$$
\begin{aligned}
& \operatorname{Parity}(-1)^{L-1}: \quad \psi_{-}^{K}(r, t) \Pi_{K-1}^{K K_{x}}(\Omega)+\psi_{+}^{K}(r, t) \Pi_{K+1}^{K K_{z}}(\Omega) \\
& \operatorname{Parity}(-1)^{L}: \quad \psi_{\circ}^{K}(r, t) \Pi_{K}^{K K_{*}}(\Omega) .
\end{aligned}
$$

One can imagine expanding an arbitrary pion field in terms of these eigenfunctions and carrying out the angular integration in (II.3). The result will be a purely radial problem, where for each value of $K, \widehat{\mathbf{L}}$ is replaced by an operator

$$
\hat{\mathbf{L}}_{K}^{\text {eff }}(r, t)=\left(\begin{array}{ccc}
\hat{\mathbf{L}}_{K}^{-} & 0 & \hat{\mathbf{L}}_{K}^{+} \\
0 & \hat{\mathbf{L}}_{K}^{\circ \circ} & 0 \\
\hat{\mathbf{L}}_{K}^{+-} & 0 & \hat{\mathbf{L}}_{K}^{++}
\end{array}\right)
$$

acting on the space

$$
\left(\begin{array}{l}
\psi_{-}^{K} \\
\psi_{0}^{K} \\
\psi_{+}^{K}
\end{array}\right)
$$

with the zeroes of course reflecting parity conservation.

Furthermore from the radial wavefunctions $\psi^{K}(r, t)$ one can construct the "effective" Green's functions $g_{K}\left(r t ; r^{\prime} t^{\prime}\right)$ associated with $\widehat{\mathbf{L}}_{K}^{\text {eff }}$; this is of course a $3 \times 3$ matrix checkered with zeroes just as in (A.7). We will find it convenient to label its matrix elements by pairs of subscripts $\left(L, L^{\prime}\right)$ that take values $(K-$ $1, K, K+1)$ instead of the usual $(1,2,3)$ or $(+, 0,-)$; thus $\mathbf{g}_{435}$, say, instead of $\left(\mathrm{g}_{4}\right)_{1,3}$.

Having defined $\mathbf{g}_{K}$ we are now in a position to write down the partial wave decomposition of the pion propagator in the unrotated soliton background. One 
finds

$$
\begin{aligned}
&\left\langle\pi^{m}\left(\vec{x}^{\prime} t^{\prime}\right) \pi^{n *}(\vec{x}, t)\right\rangle_{0}=\sum_{L L_{z} L^{\prime} L_{z}^{\prime}}\left\{Y_{L^{\prime} L_{z}^{\prime}}\left(\Omega^{\prime}\right) Y_{L L_{z}}^{*}(\Omega) \times\right. \\
&\left.\sum_{K K_{z}}\left\langle K K_{z} \mid L 1 L_{z} n\right\rangle\left\langle L^{\prime} 1 L_{z}^{\prime} m \mid K K_{z}\right\rangle g_{K L^{\prime} L}\left(r t ; r^{\prime} t^{\prime}\right)\right\}
\end{aligned}
$$

with $\vec{L}$ and $\vec{L}^{\prime}$ the incoming and outgoing pion angular momentum, respectively. This formula will presently prove very useful to us.

We now have all the machinery in place for our final result. We return to Eq. (A.5) and project the initial and final pion-baryon states onto states of definite total angular momentum $\left|J J_{x}\right\rangle$ and $\left|J^{\prime} J_{z}^{\prime}\right\rangle$, respectively; likewise the incoming and outgoing pions are restricted to partial waves $L$ and $L^{\prime}$, which we still have the freedom to specify. A short calculation making use of (A.8) then produces

$$
\begin{aligned}
\langle\pi \pi\rangle_{R \vec{I} \vec{J} L ; R^{\prime} \vec{I}^{\prime} \vec{J}^{\prime} L^{\prime}}= & \delta_{I I^{\prime}} \delta_{I_{z} I_{z}^{\prime}} \frac{\sqrt{(2 R+1)\left(2 R^{\prime}+1\right)}}{2 I+1} \\
& \times \sum_{K K_{z} m n} \sum_{x_{z}^{\prime} L_{z} L_{z} L_{z}^{\prime}}(-1)^{n-m} g_{K L^{\prime} L} \\
& \times\left\langle R L s_{z} L_{z} \mid J J_{z}\right\rangle\left\langle J^{\prime} J_{z}^{\prime} \mid R^{\prime} L^{\prime} s_{z}^{\prime} L_{z}^{\prime}\right\rangle \\
& \times\left\langle I, s_{z}-n \mid R^{\prime} 1 s_{z}^{\prime},-m\right\rangle\left\langle R 1 s_{z},-n \mid I, s_{z}-n\right\rangle \\
& \times\left\langle K K_{z} \mid L 1 L_{z} n\right\rangle\left\langle L^{\prime} 1 L_{z}^{\prime} m \mid K K_{z}\right\rangle .
\end{aligned}
$$

It is certainly not manifest from this rather unwieldy formula that total angular momentum is conserved, as of course it must be. In order to see that, it is convenient to reexpress the Clebsch-Gordon coefficients as $3 j$-symbols

$$
\left(\begin{array}{ccc}
j_{1} & j_{2} & J \\
m_{1} & m_{2} & -M
\end{array}\right)=(-1)^{j_{1}-j_{2}+M}(2 J+1)^{-1 / 2}\left\langle j_{1} j_{2} m_{1} m_{2} \mid J M\right\rangle
$$


These, in turn, can be used to construct the $6 j$-symbols via

$$
\begin{gathered}
\sum_{M_{1} M_{2} M_{3}}(-1)^{J_{1}+J_{2}+J_{3}+M_{1}+M_{2}+M_{3}}\left(\begin{array}{ccc}
J_{1} & J_{2} & j_{3} \\
M_{1} & -M_{2} & m_{3}
\end{array}\right) \\
\times\left(\begin{array}{ccc}
J_{2} & J_{3} & j_{1} \\
M_{2} & -M_{3} & m_{1}
\end{array}\right)\left(\begin{array}{ccc}
J_{3} & J_{1} & j_{2} \\
M_{3} & -M_{1} & m_{2}
\end{array}\right) \\
=\left(\begin{array}{ccc}
j_{1} & j_{2} & j_{3} \\
m_{1} & m_{2} & m_{3}
\end{array}\right)\left\{\begin{array}{lll}
j_{1} & j_{2} & j_{3} \\
J_{1} & J_{2} & J_{3}
\end{array}\right\}
\end{gathered}
$$

A short calculation making use of some standard properties of $6 j$-symbols produces

$$
\begin{aligned}
\left\langle\pi\left(r^{\prime} t^{\prime}\right) \pi(r t)\right\rangle_{R \vec{I} J_{L} ; R^{\prime} \vec{I}^{\prime} \vec{J}^{\prime} L^{\prime}} & \\
& =\delta_{I I^{\prime}} \delta_{I_{z} I_{z}} \delta_{J J^{\prime}} \delta_{J_{k} J !} \sum_{K} P_{L L^{\prime} R R^{\prime} I J K} \cdot \mathrm{g}_{K L^{\prime} L}\left(r t ; r^{\prime} t^{\prime}\right)
\end{aligned}
$$

where the $P$-symbols are defined in Eq. (II.10). Both isospin and angular momentum are now manifestly conserved. This is Eq. (II.9). 


\section{APPENDIX B: Explicit formulae for $P$-symbols}

This appendix contains explicit formulas for those $P$-symbols as defined by Eq. (II.10) which contribute to either $\pi N \rightarrow \pi N$ or $\pi N \rightarrow \pi \Delta$. The notation is $P_{L L^{\prime} R R^{\prime} I J K}$ where $L$ and $L^{\prime}$ give the orbital angular momentum of the incoming and outgoing pion, respectively; $R$ and $R^{\prime}$ denote the spin (and isospin) representation of the initial and final baryon; $I$ and $J$ are total isospin and angular momentum; and $K$ labels the conserved vector $\vec{I}$ (pion) $+\vec{L}($ pion $)$.

We consider first the process $\pi N \rightarrow \pi N$, which in our notation implies $L=$ $L^{\prime}$ and $R=R^{\prime}=\frac{1}{2}$. With two exceptions as noted below, the scattering must be $P$-wave or higher in order for the $P$-symbol not to vanish. For $L \geq 1$ the nonvanishing values of the $P$-symbols are given by: 


$$
\begin{aligned}
P_{L L \frac{1}{2} \frac{1}{2}, L-\frac{1}{2}, L-1} & =\frac{2 L-1}{3 L} \\
P_{L L \frac{1}{2} \frac{1}{2} \frac{1}{2}, L-\frac{1}{2}, L} & =\frac{L+1}{3 L} \\
P_{L L \frac{1}{2} \frac{1}{2} \frac{1}{2}, L+\frac{1}{2}, L} & =\frac{L}{3 L+3} \\
P_{L L \frac{1}{2} \frac{1}{2} \frac{1}{2}, L+\frac{1}{2}, L+1} & =\frac{2 L+3}{3 L+3} \quad(\text { also valid for } L=0) \\
P_{L L \frac{1}{2} \frac{1}{2} \frac{3}{2}, L-\frac{1}{2}, L-1} & =\frac{(2 L-1)(L-1)}{6 L(2 L+1)} \\
P_{L L \frac{1}{2} \frac{1}{2} \frac{3}{2}, L-\frac{1}{2}, L} & =\frac{2 L-1}{6 L} \\
P_{L L \frac{1}{2} \frac{1}{2} \frac{1}{2}, L-\frac{1}{2}, L+1} & =\frac{2 L+3}{4 L+2} \\
P_{L L \frac{1}{2} \frac{3}{2} \frac{3}{2}, L+\frac{1}{2}, L-1} & =\frac{2 L-1}{4 L+2} \\
P_{L L \frac{1}{2} \frac{1}{2} \frac{3}{2}, L+\frac{1}{2}, L} & =\frac{2 L+3}{6 L+6} \\
P_{L L \frac{1}{2} \frac{1}{2} \frac{3}{2}, L+\frac{1}{2}, L+1} & =\frac{(L+2)(2 L+3)}{(6 L+6)(2 L+1)} \quad(\text { also valid for } L=0)
\end{aligned}
$$




$$
\begin{aligned}
P_{L L \frac{1}{2} \frac{3}{2} \frac{1}{2}, L-\frac{1}{2}, L-1} & =\frac{\sqrt{(L+1)(2 L-1)}}{3 L} \\
P_{L L \frac{1}{2} \frac{1}{2} \frac{1}{2}, L-\frac{1}{2}, L} & =\frac{-\sqrt{(L+1)(2 L-1)}}{3 L} \\
P_{L L \frac{1}{2} \frac{8}{2} \frac{1}{2}, L+\frac{1}{2}, L} & =\frac{\sqrt{L(2 L+3)}}{3 L+3} \\
P_{L L \frac{1}{2} \frac{3}{2} \frac{1}{2}, L+\frac{1}{2}, L+1} & =\frac{-\sqrt{L(2 L+3)}}{3 L+3} \\
P_{L L \frac{1}{2} \frac{3}{2} \frac{3}{2}, L-\frac{1}{2}, L-1} & =\frac{4(L-1)}{3 L(2 L+1)} \sqrt{\frac{(2 L-1)(L+1)}{10}} \\
P_{L L \frac{1}{2} \frac{3}{2} \frac{3}{2}, L-\frac{1}{2}, L} & =\frac{(L+4)}{3 L} \sqrt{\frac{2 L-1}{10(L+1)}} \\
P_{L L \frac{1}{2} \frac{3}{2} \frac{3}{2}, L-\frac{1}{2}, L+1} & =-\frac{2 L+3}{2 L+1} \sqrt{\frac{2 L-1}{10(L+1)}} \\
P_{L L \frac{1}{2} \frac{3}{2} \frac{3}{2}, L+\frac{1}{2}, L-1} & =\frac{(2 L-1)}{2 L+1} \sqrt{\frac{2 L+3}{10 L}} \\
P_{L L \frac{1}{2} \frac{3}{2} \frac{3}{2}, L+\frac{1}{2}, L} & =-\frac{L-3}{3 L+3} \sqrt{\frac{2 L+3}{10 L}} \\
P_{L L \frac{1}{2} \frac{3}{2} \frac{3}{2}, L+\frac{1}{2}, L+1} & =-\frac{4(L+2)}{(3 L+3)(2 L+1)} \sqrt{\frac{L(2 L+3)}{10}}
\end{aligned}
$$

The final possibility is $\pi N \rightarrow \pi \Delta$ with $L=L^{\prime} \pm 2$. Now $L=0$ is allowed, and we have: 


$$
\begin{aligned}
& P_{L, L+2, \frac{1}{2} \frac{8}{2} \frac{1}{2}, L+\frac{1}{2}, L+1}=-\sqrt{\frac{2 L+3}{3(L+1)}} \\
& P_{L, L+2, \frac{1}{2} \frac{8}{2} \frac{3}{2}, L+\frac{1}{2}, L+1}=\sqrt{\frac{2 L+3}{30(L+1)}} \\
& P_{L+2, L \frac{1}{2} \frac{8}{2} \frac{1}{2}, L+\frac{3}{2}, L+1}=\sqrt{\frac{2 L+3}{3(L+2)}} \\
& P_{L+2, L \frac{1}{2} \frac{8}{2} \frac{8}{2}, L+\frac{8}{2}, L+1}=-\sqrt{\frac{2 L+3}{30(L+2)}}
\end{aligned}
$$

All other $P$-symbols with $R=\frac{1}{2}$ and $R^{\prime}=\frac{1}{2}$ or $\frac{3}{2}$ vanish. 


\section{APPENDIX C: Further Details on Skyrme Model Equations}

In this appendix we give some further details concerning the differential equations (III.12a) and (III.12b). We will express our results in terms of the dimensionless variables $\tilde{r}=e f_{\pi} r$ and $\tilde{m}=m /\left(e f_{\pi}\right)$.

We consider the ' $1 \times 1$ ' case (III.12a) first. After multiplying through by $2 F^{2} / \sin ^{2} F$ the equation turns out to be

$$
\begin{aligned}
& \left(1+\frac{4 \sin ^{2} F}{\tilde{r}^{2}}\right) \frac{d^{2}}{d \tilde{r}^{2}} \psi_{0}^{K}+\left[\frac{2}{\tilde{r}}+F^{\prime}\left(2 \cot F-\frac{2}{F}+\frac{8 \sin 2 F}{\tilde{r}^{2}}-\frac{8 \sin ^{2} F}{F^{2}}\right)\right] \frac{d}{d \tilde{r}} \psi_{0}^{K} \\
& -\left\{\frac{K(K+1)}{\tilde{r}^{2}}\left[1+4\left(F^{\prime}\right)^{2}\right]-\frac{2}{\tilde{r}^{2}}+F^{\prime \prime}\left(\frac{1}{F}+\frac{4 \sin ^{2} F}{\tilde{r}^{2} F}\right)+\frac{2 F^{\prime}}{\tilde{r} F}\right. \\
& +\left(F^{\prime}\right)^{2}\left(-\frac{2}{F^{2}}-\frac{8 \sin ^{2} F}{\tilde{r}^{2} F^{2}}-\frac{8}{\tilde{r}^{2}}+\frac{2 \cot F}{F}+\frac{8 \sin 2 F}{\tilde{r}^{2} F}\right) \\
& \left.+\frac{F}{\tilde{r}^{2} \sin ^{2} F}\left[\tilde{r}^{2} F^{\prime \prime}+\ldots\right]-\omega^{2}\left[1+4\left(F^{\prime}\right)^{2}+4 \frac{\sin ^{2} F}{\tilde{r}^{2}}\right]\right\} \psi_{0}^{K}=0
\end{aligned}
$$

The term $\left[\widetilde{r}^{2} F^{\prime \prime}+\ldots\right]$ denotes the defining equation for $F$, Eq. (III.4), which of course is identically zero. Near the origin the regular solution satisfies $\psi_{0}^{K} \sim$ $\tilde{r}^{K-1}\left[\pi-\alpha \tilde{r}+O\left(\tilde{r}^{2}\right)\right]$ where $\alpha \equiv\left|F^{\prime}(0)\right| \cong 1$; from this we obtain the initial conditions needed to carry out the numerical integration.

The ' $2 \times 2$ ' case (III.12b) is more complicated. It is convenient to change variables to

$$
\psi_{1}^{K}=\frac{-\sqrt{K} \psi_{-}^{K}+\sqrt{K+1} \psi_{+}^{K}}{\sqrt{2 K+1}}
$$

and

$$
\psi_{2}^{K}=\frac{\sqrt{K+1} \psi_{-}^{K}+\sqrt{K} \psi_{+}^{K}}{\sqrt{2 K+1}}
$$


We then need to solve the coupled linear equations

$$
\begin{aligned}
& -2 F^{4} \tilde{r}^{2}\left(\tilde{r}^{2}+8 \sin ^{2} F\right) \frac{d^{2}}{d \tilde{r}^{2}} \psi_{1}^{K}-4 F^{4} \tilde{r}^{2}\left(\tilde{r}+4 F^{\prime} \sin 2 F\right) \frac{d}{d \tilde{r}} \psi_{1}^{K}+ \\
& \left\{2 F^{4}[2 \cos 2 F+K(K+1)]\left[\tilde{r}^{2}+4 \sin ^{2} F\right]\right. \\
& +2 F^{4}\left[4 \sin ^{2} 2 F-8 \tilde{r}^{2} F^{\prime \prime} \sin 2 F+\tilde{m}^{2} \tilde{r}^{4} \cos F-8 \tilde{r}^{2}\left(F^{\prime}\right)^{2} \cos 2 F\right] \\
& \left.-2 \tilde{r}^{2} F^{4}\left(\tilde{r}^{2}+8 \sin ^{2} F\right) \omega^{2}\right\} \psi_{1}^{K}-8 \sqrt{K(K+1)} F^{3} F^{\prime} \tilde{r}^{2} \sin ^{2} F \frac{d}{d \tilde{r}} \psi_{2}^{K} \\
& +\left\{F ^ { 2 } \sqrt { K ( K + 1 ) } \operatorname { s i n } ^ { 2 } F \left\{16 F \sin 2 F-16 \tilde{r}^{2} F F^{\prime \prime}\right.\right. \\
& \left.\left.+4 r^{2} F \cot F\left[1-4\left(F^{\prime}\right)^{2}\right]+8 \tilde{r}^{2}\left(F^{\prime}\right)^{2}\right\}\right\} \psi_{2}^{K}=0
\end{aligned}
$$

and 


$$
\begin{aligned}
& -2 F^{2} \tilde{r}^{2}\left(\tilde{r}^{2}+4 \sin ^{2} F\right) \frac{d^{2}}{d \tilde{r}^{2}} \psi_{2}^{K}+ \\
& {\left[-4 F^{2} \tilde{r}^{3}+\tilde{r}^{2} F^{\prime}\left(-16 F^{2} \sin 2 F-4 F^{2} \cot F^{2}+16 F \sin ^{2} F+4 \tilde{r}^{2} F\right)\right] \frac{d}{d \tilde{r}} \psi_{2}^{K}} \\
& +\left\{K(K+1) F^{2}\left\{8 \sin ^{2} F+2 \tilde{r}^{2}\left[1+4\left(F^{\prime}\right)^{2}\right]\right\}\right. \\
& +2 \tilde{r}^{2}\left[-8 F^{2}\left(F^{\prime}\right)^{2}-2 F^{2}+4 F \sin ^{2} F F^{\prime \prime}+8 F \sin 2 F\left(F^{\prime}\right)^{2}+F \tilde{r}\left(2 F^{\prime}+\tilde{r} F^{\prime \prime}\right)\right. \\
& \left.+2 F \cot F^{2}\left(F^{\prime}\right)^{2}-8 \sin ^{2} F\left(F^{\prime}\right)^{2}-2 \tilde{r}^{2}\left(F^{\prime}\right)^{2}\right]-\frac{2 \tilde{r}^{2} F^{3}}{\sin ^{2} F}\left[\tilde{r}^{2} F^{\prime \prime}+\ldots\right] \\
& \left.-2 \omega^{2} F^{2} \tilde{r}^{2}\left[4 \sin ^{2} F+4 \tilde{r}^{2}\left(F^{\prime}\right)^{2}+\tilde{r}^{2}\right]\right\} \psi_{2}^{K}+8 \sqrt{K(K+1)} \tilde{r}^{2} F^{3} F^{\prime} \frac{d}{d \tilde{r}} \psi_{1}^{K} \\
& +\left\{8 F^{3} \sqrt{K(K+1)}\left[2 \sin 2 F-\tilde{r}^{2} F^{\prime \prime}+\frac{\tilde{r}^{2}}{2} \cot F\right]\right\} \psi_{1}^{K}=0
\end{aligned}
$$

where we have multiplied through by $-4 \widetilde{r}^{4} F^{4}$ and $-4 \widetilde{r}^{4} F^{4} / \sin ^{2} F$, respectively. (The advantage of the change of variables is that each of these equations is second-order in only one variable.) We are relieved to find that, in the case of massless pions, Eqs. (C.1)-(C.3) are in agreement with Ref. 22. One can check that the translational mode (III.19b) is a zero-energy solution of Eq. (C.3) when $K=1$.

Now there are two regular solutions near the origin:

$$
\left(\begin{array}{c}
\psi_{1}^{K} \\
\psi_{2}^{K}
\end{array}\right) \sim\left(\begin{array}{c}
\tilde{r}^{K \pm 1}\left[1+O\left(\tilde{r}^{2}\right)\right] \\
B_{ \pm} \tilde{r}^{K \pm 1}\left[\frac{\pi}{\alpha \tilde{f}}-1+O(\tilde{r})\right]
\end{array}\right)
$$

where

$$
B_{+}=\sqrt{\frac{K}{K+1}} \frac{1+2 \alpha^{2} K+10 \alpha^{2}}{-1+2 \alpha^{2} K-4 \alpha^{2}}
$$


and

$$
B_{-}=\sqrt{\frac{K+1}{K}}
$$

It is straightforward to integrate these equations numerically out to large $\tilde{r}$, reconstruct $\psi_{ \pm}^{K}$, and extract the phase shifts. The development then proceeds as outlined in Sec. III.1. 


\section{APPENDIX D: Explicit Formulae for $S U(3)$ Scattering Coefficients}

In this appendix we present explicit formulae for the group-theoretic coefficients multiplying the eight reduced amplitudes in the $S U(3)$ scattering formula, Eq. (IV.10). For fixed baryon representations, these depend only on the total meson-baryon representation $R_{\text {tot }}$ and the total angular momentum $J$. We will restrict ourselves to the physically relevant cases when the initial baryon is in the spin- $\frac{1}{2}$ octet, and the final baryon is in either the octet or the spin- $\frac{3}{2}$ decuplet.

Table III presents the coefficients of the reduced amplitudes for the case when the initial and final baryon are both in the octet. The relevant decomposition is

$$
8 \times 8=27+\overline{10}+10+8_{1}+8_{2}+1
$$

where (following DeSwart ${ }^{|30|}$ ) the $8_{1}$ and $8_{2}$ are synonymous with $8_{\text {sym }}$ and $8_{\text {antisym }}$, respectively.

Table IV gives the prescription for $\pi N$ elastic scattering in the 3-flavor formalism. These coefficients can be directly compared to their 2-flavor cousins presented in Appendix B.

Table $\mathrm{V}$ presents the relevant coefficients when the initial and final baryons are in the octet and decuplet, respectively, and when the initial and final meson partial waves are the same $\left(L=L^{\prime}\right)$. The relevant decomposition is

$$
10 \times 8=35+27+10+8 .
$$

Table VI lists the coefficients for the analogous $8 \times 8 \longrightarrow 10 \times 8$ processes when $\left|L-L^{\prime}\right|=2$. Note that these coefficients all multiply the single contributing reduced amplitude $s_{K L^{\prime} L}^{\text {pion }}$, where $K=\left(L+L^{\prime}\right) / 2$. Thus, to leading order in $1 / N$, all these processes are predicted to be strictly proportional to one another; this is a model-independent result. 


\begin{tabular}{|c|c|c|c|c|c|c|c|c|}
\hline$R_{t o t}$ & $8_{L-1, L, L}^{\text {pion }}$ & $8_{L L L}^{p i o n}$ & $\mathrm{~s}_{L+1, L, L}^{\text {pion }}$ & $\mathbf{s}_{L L}^{e t a}$ & $\mathbf{s}_{L-\frac{1}{2}, L}^{k a o n}$ & $8_{L+\frac{1}{2}}^{k a o n}$ & $\mathbf{8}_{L-\frac{1}{2}, L}^{k-b a r}$ & $\mathbf{s}_{L+\frac{1}{2}}^{k-b a r}$ \\
\hline$\langle 27 \mid 27\rangle$ & $\frac{2}{135} \frac{(2 L-1)(4 L-3)}{L(2 L+1)}$ & $\frac{2}{195} \frac{7 L-3}{L}$ & $\frac{4}{27} \frac{2 L+3}{2 L+1}$ & $\frac{2}{15}$ & $\frac{4}{27} \frac{2 L-1}{2 L+1}$ & $\frac{16}{27} \frac{L+1}{2 L+1}$ & $\frac{1}{135} \frac{14 L-1}{2 L+1}$ & $\frac{16}{135} \frac{L+1}{2 L+1}$ \\
\hline$\langle\overline{\mathbf{1 0}} \mid \overline{\mathbf{1 0}}\rangle$ & $\frac{1}{15} \frac{2 L-1}{L}$ & $\frac{1}{15} \frac{L+1}{L}$ & 0 & $\frac{1}{5}$ & $\frac{2}{5}$ & 0 & $\frac{1}{15} \frac{2 L-1}{2 L+1}$ & $\frac{4}{15} \frac{L+1}{2 L+1}$ \\
\hline$\langle\mathbf{1 0} \mid \mathbf{1 0}\rangle$ & $\frac{2}{15} \frac{(2 L-1)(L-1)}{L(2 L+1)}$ & $\frac{2}{15} \frac{2 L-1}{L}$ & $\frac{2}{5} \frac{2 L+3}{2 L+1}$ & 0 & 0 & 0 & $\frac{1}{15} \frac{2 L-1}{2 L+1}$ & $\frac{4}{15} \frac{L+1}{2 L+1}$ \\
\hline$\left\langle\mathbf{8}_{1} \mid \mathbf{8}_{1}\right\rangle$ & $\frac{3}{20} \frac{2 L-1}{L}$ & $\frac{3}{20} \frac{L+1}{L}$ & 0 & $\frac{1}{20}$ & 0 & 0 & $\frac{1}{10} \frac{4 L-1}{2 L+1}$ & $\frac{3}{5} \frac{L+1}{2 L+1}$ \\
\hline$\left\langle\mathbf{8}_{2} \mid \mathbf{8}_{2}\right\rangle$ & $\frac{2 L-1}{12 L}$ & $\frac{L+1}{12 L}$ & 0 & $\frac{1}{4}$ & 0 & 0 & $\frac{1}{6} \frac{4 L+1}{2 L+1}$ & $\frac{1}{3} \frac{L+1}{2 L+1}$ \\
\hline$\left\langle\mathbf{8}_{1} \mid \mathbf{8}_{2}\right\rangle$ & $\frac{2 L-1}{L \sqrt{80}}$ & $\frac{L+1}{L \sqrt{80}}$ & 0 & $\frac{-1}{\sqrt{80}}$ & 0 & 0 & $\frac{1}{2 \sqrt{5}(2 L+1)}$ & $\frac{-(L+1)}{\sqrt{5}(2 L+1)}$ \\
\hline$\langle\mathbf{1} \mid \mathbf{1}\rangle$ & 0 & 0 & 0 & 0 & 0 & 0 & 1 & 0 \\
\hline
\end{tabular}

\begin{tabular}{|c|c|c|c|c|c|c|c|c|}
\hline \multicolumn{8}{|c|}{$J=L+\frac{1}{2}$} \\
\hline$R_{\text {tot }}$ & $\mathrm{s}_{L-1, L, L}^{\text {pion }}$ & $\mathrm{s}_{L L L}^{\text {pion }}$ & $\mathrm{s}_{L+1, L, L}^{\text {pion }}$ & $\mathrm{s}_{L L}^{\text {eta }}$ & $\mathrm{s}_{L-\frac{1}{2}, L}^{\text {kaon }}$ & $\mathrm{s}_{L+\frac{1}{2}}^{\text {kaon }}$ & $\mathrm{s}_{L-\frac{1}{2}, L}^{k-b a r}$ & $\mathrm{~s}_{L+\frac{1}{2}}^{k-b a r}$ \\
\hline$\langle\mathbf{2 7} \mid \mathbf{2 7}\rangle$ & $\frac{4}{27} \frac{2 L-1}{2 L+1}$ & $\frac{2}{135} \frac{7 L+10}{L+1}$ & $\frac{2}{135} \frac{(2 L+3)(4 L+7)}{(L+1)(2 L+1)}$ & $\frac{2}{15}$ & $\frac{16}{27} \frac{L}{2 L+1}$ & $\frac{4}{27} \frac{2 L+3}{2 L+1}$ & $\frac{16}{135} \frac{L}{2 L+1}$ & $\frac{1}{135} \frac{14 L+15}{2 L+1}$ \\
$\langle\overline{10} \mid \overline{10}\rangle$ & 0 & $\frac{1}{15} \frac{L}{L+1}$ & $\frac{1}{15} \frac{2 L+3}{L+1}$ & $\frac{1}{5}$ & 0 & $\frac{2}{5}$ & $\frac{4}{15} \frac{L}{2 L+1}$ & $\frac{1}{15} \frac{2 L+3}{2 L+1}$ \\
$\langle 10 \mid 10\rangle$ & $\frac{2}{5} \frac{2 L-1}{2 L+1}$ & $\frac{2}{15} \frac{2 L+3}{L+1}$ & $\frac{2}{15} \frac{(L+2)(2 L+3)}{(L+1)(2 L+1)}$ & 0 & 0 & 0 & $\frac{4}{15} \frac{L}{2 L+1}$ & $\frac{1}{15} \frac{2 L+3}{2 L+1}$ \\
$\left\langle 8_{1} \mid 8_{1}\right\rangle$ & 0 & $\frac{3}{20} \frac{L}{L+1}$ & $\frac{3}{20} \frac{2 L+3}{L+1}$ & $\frac{1}{20}$ & 0 & 0 & $\frac{3}{5} \frac{L}{2 L+1}$ & $\frac{1}{10} \frac{4 L+5}{2 L+1}$ \\
$\left\langle 8_{2} \mid 8_{2}\right\rangle$ & 0 & $\frac{L}{12(L+1)}$ & $\frac{2 L+3}{12(L+1)}$ & $\frac{1}{4}$ & 0 & 0 & $\frac{1}{3} \frac{L}{2 L+1}$ & $\frac{1}{6} \frac{4 L+3}{2 L+1}$ \\
$\left\langle 8_{1} \mid 8_{2}\right\rangle$ & 0 & $\frac{L}{(L+1) \sqrt{80}}$ & $\frac{2 L+3}{(L+1) \sqrt{80}}$ & $\frac{-1}{\sqrt{80}}$ & 0 & 0 & $\frac{-L}{\sqrt{5}(2 L+1)}$ & $\frac{-1}{2 \sqrt{5}(2 L+1)}$ \\
$\langle 1 \mid 1\rangle$ & 0 & 0 & 0 & 0 & 0 & 0 & 0 & 1 \\
\hline
\end{tabular}

Table III 
$\pi N \longrightarrow \pi N$

\begin{tabular}{|c|c|c|c|c|c|c|c|c|c|}
\hline $\mathbf{s}_{L-1, L, L}^{\text {pion }}$ & $\mathrm{s}_{L L L}^{\text {pion }}$ & $\mathbf{8}_{L+1, L, L}^{\text {pion }}$ & $8_{L L}^{e t a}$ & $\mathrm{~B}_{L-\frac{1}{2}, L}^{k a o n}$ & $\mathbf{s}_{L+\frac{1}{2}}^{k a o n}$ & $\mathbf{8}_{L-\frac{1}{2}, L}^{k-b a r}$ & $\mathbf{8}_{L+\frac{1}{2}}^{k-b a r}$ & $\mathrm{~J}$ & I \\
\hline$\frac{(2 L-1)(49 L+24)}{135 L(2 L+1)}$ & $\frac{25 L+24}{135 L}$ & $\frac{2 L+3}{135(2 L+1)}$ & $\frac{1}{15}$ & $\frac{58 L+25}{270(2 L+1)}$ & $\frac{4(L+1)}{135(2 L+1)}$ & $\frac{104 L+35}{270(2 L+1)}$ & $\frac{17(L+1)}{135(2 L+1)}$ & $L-\frac{1}{2}$ & $\frac{1}{2}$ \\
\hline$\frac{2 L-1}{135(2 L+1)}$ & $\frac{25 L+1}{135(L+1)}$ & $\frac{(2 L+3)(49 L+25)}{135(2 L+1)(L+1)}$ & $\frac{1}{15}$ & $\frac{4 L}{135(2 L+1)}$ & $\frac{58 L+33}{270(2 L+1)}$ & $\frac{17 L}{135(2 L+1)}$ & $\frac{104 L+69}{270(2 L+1)}$ & $L+\frac{1}{2}$ & $\frac{1}{2}$ \\
\hline$\frac{(2 L-1)(13 L-12)}{135 L(2 L+1)}$ & $\frac{25 L-12}{135 L}$ & $\frac{37}{135} \frac{2 L+3}{2 L+1}$ & $\frac{1}{15}$ & $\frac{2(2 L-1)}{27(2 L+1)}$ & $\frac{8(L+1)}{27(2 L+1)}$ & $\frac{16 L-5}{135(2 L+1)}$ & $\frac{26(L+1)}{135(2 L+1)}$ & $L-\frac{1}{2}$ & $\frac{3}{2}$ \\
\hline$\frac{37}{135} \frac{2 L-1}{2 L+1}$ & $\frac{25 L+37}{135(L+1)}$ & $\frac{(2 L+3)(13 L+25)}{135(2 L+1)(L+1)}$ & $\frac{1}{15}$ & $\frac{8 L}{27(2 L+1)}$ & $\frac{2(2 L+3)}{27(2 L+1)}$ & $\frac{26 L}{135(2 L+1)}$ & $\frac{16 L+21}{135(2 L+1)}$ & $L+\frac{1}{2}$ & $\frac{3}{2}$ \\
\hline
\end{tabular}

\section{Table IV}

$J=L-\frac{1}{2}$

\begin{tabular}{|c|c|c|c|c|c|c|c|c|}
\hline$R_{\text {tot }}$ & $\mathbf{p}_{L-1, L, L}^{\text {pion }}$ & $e_{L L L}^{\text {pion }}$ & $\|_{L+1, L, L}^{\text {sion }}$ & $\mathbf{s}_{L}^{e t a t}$ & $\boldsymbol{\theta}_{L-\frac{1}{2}, L}^{\text {kaon }}$ & $a_{L+\frac{1}{1}, L}^{\text {kean }}$ & $\boldsymbol{z}_{L-\frac{1}{2}, L}^{k-b a r}$ & $a_{L+\frac{1}{2}, L}^{k-\text {-ar }}$ \\
\hline$\langle 27 \mid \mathbf{2 7}\rangle \mid$ & $\frac{(3-4 L) \sqrt{10(L+1)(2 L-1)}}{136 L(2 L+1)}$ & $\frac{-(L+6)}{(135 L} \sqrt{\frac{(2 L L-1)}{2(L+1)}}$ & $\frac{2 L+3}{27(2 L+1)} \sqrt{\frac{5(2 L-1)}{2(L+1)}}$ & 0 & $\frac{-2 \sqrt{10(2 L-1)(L+1)}}{27(3 L+1)}$ & $\frac{2 \sqrt{20(2 L-1)(L+1)}}{27(2 L+1)}$ & $\frac{2 \sqrt{(2 L-1)(L+1) / 10}}{27(2 L+1)}$ & $\frac{-2 \sqrt{(2 L-1)(L+1) / 10}}{37(2 L+1)}$ \\
\hline$\langle 10 \mid 10\rangle\}$ & $\frac{2(1-L) \sqrt{(2 L-1)(L+1) / 5}}{3 L(2 L+1)}$ & $\frac{-(L+4)}{\delta L} \sqrt{\frac{2 L-1}{\delta(L+1)}}$ & $\frac{2 L+3}{2(2 L+1)} \sqrt{\frac{2 L-1}{3(L+1)}}$ & 0 & 0 & 0 & $\frac{-\sqrt{(2 L-1)(L+1) / 5}}{3(2 L+1)}$ & $\frac{\sqrt{(2 L-1)(L+1) / 5}}{3(2 L+1)}$ \\
\hline$\left\langle 8 \mid 8_{1}\right\rangle$ & $\frac{-\sqrt{(L+1)(2 L-1) / 10}}{2 L}$ & $\frac{\sqrt{(L+1)(2 L-1) / 10}}{2 L}$ & 0 & 0 & 0 & 0 & $\frac{-\sqrt{(L+1)(2 L-1) / 10}}{2 L+1}$ & $\frac{\sqrt{(L+1)(2 L-1) / 30}}{2 L+1}$ \\
\hline$\left\langle 8 \mid 8_{3}\right\rangle$ & $\frac{-\sqrt{2(L+1)(2 L-1)}}{12 L}$ & $\frac{\sqrt{2(L+1)(2 L-1)}}{12 L}$ & o & 0 & 0 & 0 & $\frac{\sqrt{2(L+1)(2 L-1)}}{6(2 L+1)}$ & $\frac{-\sqrt{2(L+1)(2 L-1)}}{6(2 L+1)}$ \\
\hline
\end{tabular}

$$
J=L+\frac{1}{2}
$$

\begin{tabular}{|c|c|c|c|c|c|c|c|c|}
\hline$R_{\text {tot }}$ & $2_{L-1, L, L}^{\text {pion }}$ & $\Delta_{L L L}^{\text {pion }}$ & $\mathbf{s}_{L+1, L, L}^{\text {nion }}$ & $\boldsymbol{s}_{L}^{e t a}$ & $\mathbf{s}_{L-\frac{1}{2}, L}^{k e o n}$ & $\underbrace{k \text { aon }}_{L+b, L}$ & $\mathrm{~s}_{L-1, L}^{k-b a r}$ & $\boldsymbol{e}_{L+\frac{1}{2}, L}^{k-b a r}$ \\
\hline$\langle 27 \mid \mathbf{2 7}\rangle$ & $\frac{1-2 L}{27(2 L+1)} \sqrt{\frac{5(2 L+3)}{2 L}}$ & $\frac{L-5}{135(L+1)} \sqrt{\frac{5(2 L+3)}{2 L}}$ & $\frac{2(4 L+7) \sqrt{3 L(2 L+3) / 2}}{183(L+1)(2 L+1)}$ & 0 & $\frac{-2 \sqrt{10 L(2 L+3)}}{27(2 L+1)}$ & $\frac{2 \sqrt{10 L(2 L+3)}}{27(2 L+1)}$ & $\frac{2 \sqrt{L(2 L+3) / 10}}{27(2 L+1)}$ & $\frac{-2 \sqrt{L(2 L+3) / 10}}{27(2 L+1)}$ \\
\hline$\langle 10 \mid 10\rangle$ & $\frac{1-2 L}{3(2 L+1)} \sqrt{\frac{(2 L+3)}{5 L}}$ & $\frac{L-3}{6(L+1)} \sqrt{\frac{2 L+3}{6 L}}$ & $\frac{2(L+2) \sqrt{L(2 L+3) / 5}}{3(L+1)(2 L+1)}$ & 0 & 0 & 0 & $\frac{-\sqrt{L(2 L+3) / 5}}{3(2 L+1)}$ & $\frac{\sqrt{L(2 L+3) / 5}}{3(2 L+1)}$ \\
\hline$\left\langle 8 \mid 8_{1}\right\rangle$ & 0 & $\frac{-\sqrt{L(2 L+3) / 10}}{2(L+1)}$ & $\frac{\sqrt{L(2 L+3) / 10}}{2(L+1)}$ & 0 & 0 & 0 & $\frac{-\sqrt{L(2 L+3) / 10}}{2 L+1}$ & $\frac{\sqrt{L(2 L+3) / 10}}{2 L+1}$ \\
\hline$\left\langle 8 \mid B_{2}\right\rangle$ & 0 & $\frac{-\sqrt{2 L(2 L+3)}}{12(L+1)}$ & $\frac{\sqrt{2 L(2 L+3)}}{12(L+1)}$ & 0 & 0 & 0 & $\frac{\sqrt{2 L(2 L+3)}}{6(2 L+1)}$ & $\frac{-\sqrt{2 L(2 L+3)}}{6(2 L+1)}$ \\
\hline
\end{tabular}

Table V 


$$
J=L+\frac{1}{2}
$$

\begin{tabular}{|c|c|}
\hline$R_{\text {tot }}$ & $s_{L+1, L+2, L}^{\text {pion }}$ \\
\hline$\langle 27 \mid 27\rangle$ & $-\frac{1}{9} \sqrt{\frac{2 L+3}{30(L+1)}}$ \\
$\langle 10 \mid 10\rangle$ & $-\sqrt{\frac{2 L+3}{60(L+1)}}$ \\
$\left\langle 8 \mid 8_{1}\right\rangle$ & $\frac{3}{2} \sqrt{\frac{2 L+3}{30(L+1)}}$ \\
$\left\langle 8 \mid 8_{2}\right\rangle$ & $\frac{1}{4} \sqrt{\frac{2(2 L+3)}{3(L+1)}}$ \\
\hline
\end{tabular}

$$
J=L+\frac{3}{2}
$$

\begin{tabular}{|c|r|}
\hline$R_{\text {tot }}$ & $s_{L+1, L, L+2}^{\text {pion }}$ \\
\hline$\langle 27 \mid 27\rangle$ & $\frac{1}{9} \sqrt{\frac{2 L+3}{30(L+1)}}$ \\
$\langle 10 \mid 10\rangle$ & $\sqrt{\frac{2 L+3}{60(L+1)}}$ \\
$\left\langle 8 \mid 8_{1}\right\rangle$ & $-\frac{3}{2} \sqrt{\frac{2 L+3}{30(L+1)}}$ \\
$\left\langle 8 \mid 8_{2}\right\rangle$ & $-\frac{1}{4} \sqrt{\frac{2(2 L+3)}{3(L+1)}}$ \\
\hline
\end{tabular}

Table VI 


\section{REFERENCES}

1. M. P. Mattis and M. Karliner, Phys. Rev. D31 (1985) 2833.

2. M. P. Mattis and M. Peskin, Phys. Rev. D32 (1985) 58.

3. M. P. Mattis, Aspects of Meson-Skyrmion Scattering, SLAC-PUB. 3795, September 1985, to appear in S. Brodsky and E. Moniz, eds., Proceedings of the 1985 ITP Workshop on Nuclear Chromodynamics, World Scientific Press.

4. Karliner and Mattis, Hadron Dynamics in the 3-Flavor Skyrme Model, SLAC-PUB 3796.

5. T. H. R. Skyrme Proc. Roy. Soc.A260 (1961) 127.

6. T. H. R. Skyrme, Nucl. Phys. 31 (1962) 556.

7. D. Finkelstein and J. Rubinstein, J. Math. Phys. 9, 1762 (1968).

8. E. Witten, Nucl. Phys. B223 (1983) 422; ibid. 433.

9. S. Coleman, J. Wess, B. Zumino, Phys. Rev. 177 (1969) 2239; C. Callan, S. Coleman, J. Wess, B. Zumino, ibid. 2247.

10. S. Weinberg, Phys. Rev. Lett. 17, 168 (1966).

11. H. Schnitzer, Phys. Lett. 139B, 217 (1984); Brandeis preprint BRX-TH179, April 1985.

12. G. H. Derrick, J. Math. Phys. 5 (1964) 1252.

13. G. Adkins, C. Nappi, and E. Witten, Nucl. Phys. B228, 552 (1983).

14. D. M. Manley, R. A. Arndt, Y. Goradia, and V. L. Teplitz, Phys. Rev. D30, 904 (1984).

15. G. 't Hooft, Nucl. Phys. B72 (1974) 461; Nucl. Phys. B75 (1974) 461. 
16. E. Witten Nucl. Phys. B160 57 (1979).

17. A. Manohar, Nucl. Phys. B248 19 (1984).

18. A. Hayashi, G. Eckart, G. Holzwarth, and H. Walliser, Phys. Lett. 147B, 5 (1984).

19. I. Zahed, U.-G. Meissner, and U. B. Kaulfuss, Nucl. Phys. A426, 525 (1984).

20. A. Hayashi and G. Holzwarth, Phys. Lett. 140B, 175 (1984).

21. J. D. Breit and C. R. Nappi, Phys. Rev. Lett. 53, 889 (1984).

22. H. Walliser and G. Eckart, Nucl. Phys. A429, 514 (1984).

23. See, for example, J. R. Taylor, Scattering Theory. (Wiley, N. Y., 1972).

24. G. Höhler, F. Kaiser, R. Koch, and E. Pietarinen, Handbook of PionNucleon Scattering (Fachinformationszentrum, Karlsruhe, 1979), Physik Daten No. 12-7.

25. M. Messiah, Quantum Mechanics, Amsterdam, North Holland, 1961.

26. N. Isgur and G. Karl, Phys. Rev. D18 (1978) 4187; ibid, 19 (1979) 2653; 20 (1979) 1191.

27. E. Guadagnini, Nucl. Phys. B236 (1984) 35.

28. J. Wess and B. Zumino, Phys. Lett. 37B (1971) 95.

29. C. G. Callan and I. Klebanov, Bound state approach to strangeness in the Skyrme model, Princeton preprint Print-85-0733, June 1985.

30. J. J. deSwart, Rev. Mod. Phys. 35 (1963) 916; reprinted in M. Gell-Mann and Y. Ne'eman, The Eightfold Way, New York 1964, p. 120.

31. K. Nakajima et al., Phys. Lett. 112B, 80 (1982). 


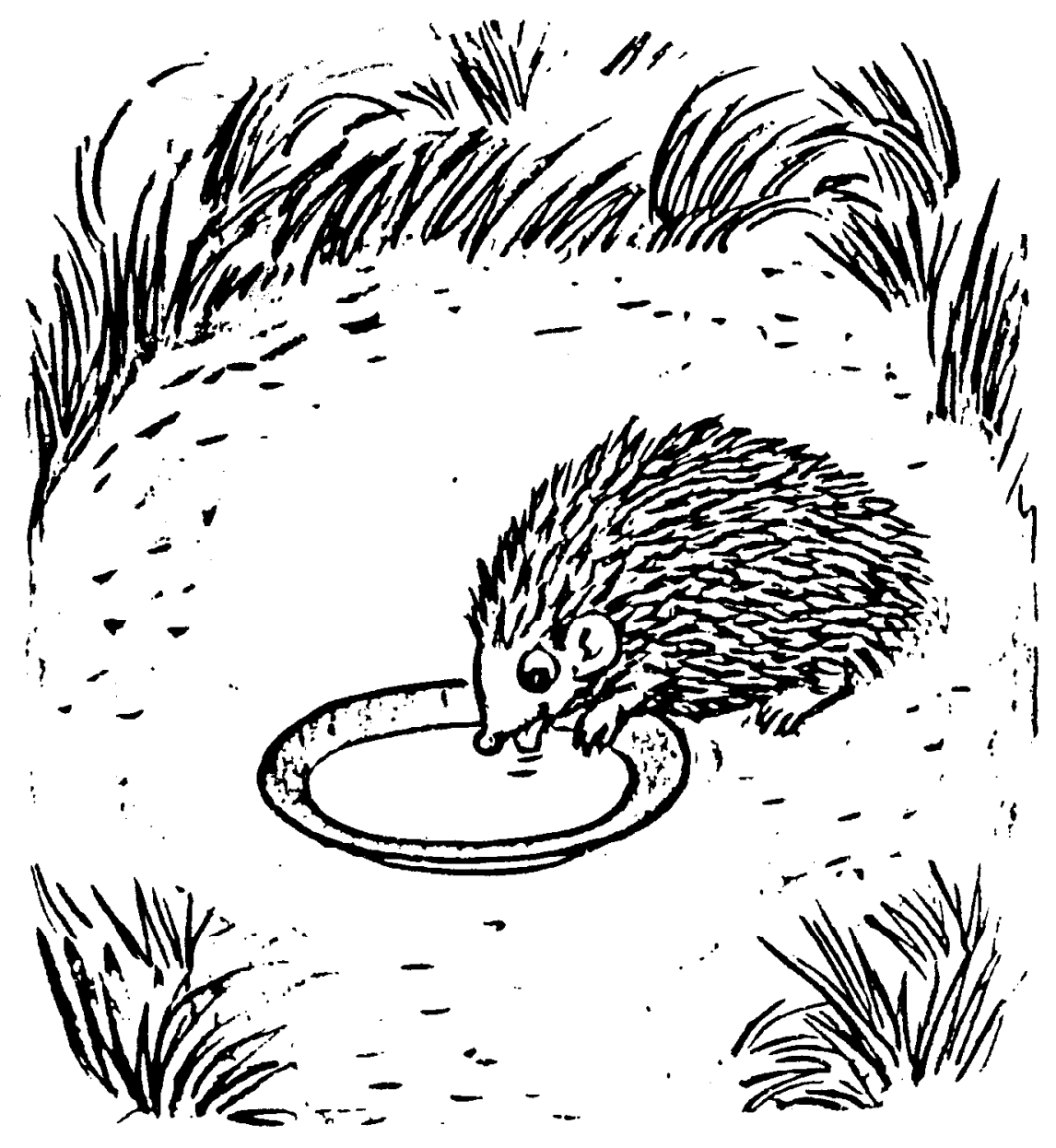

Fig. 27. An unrotated hedgehog. 


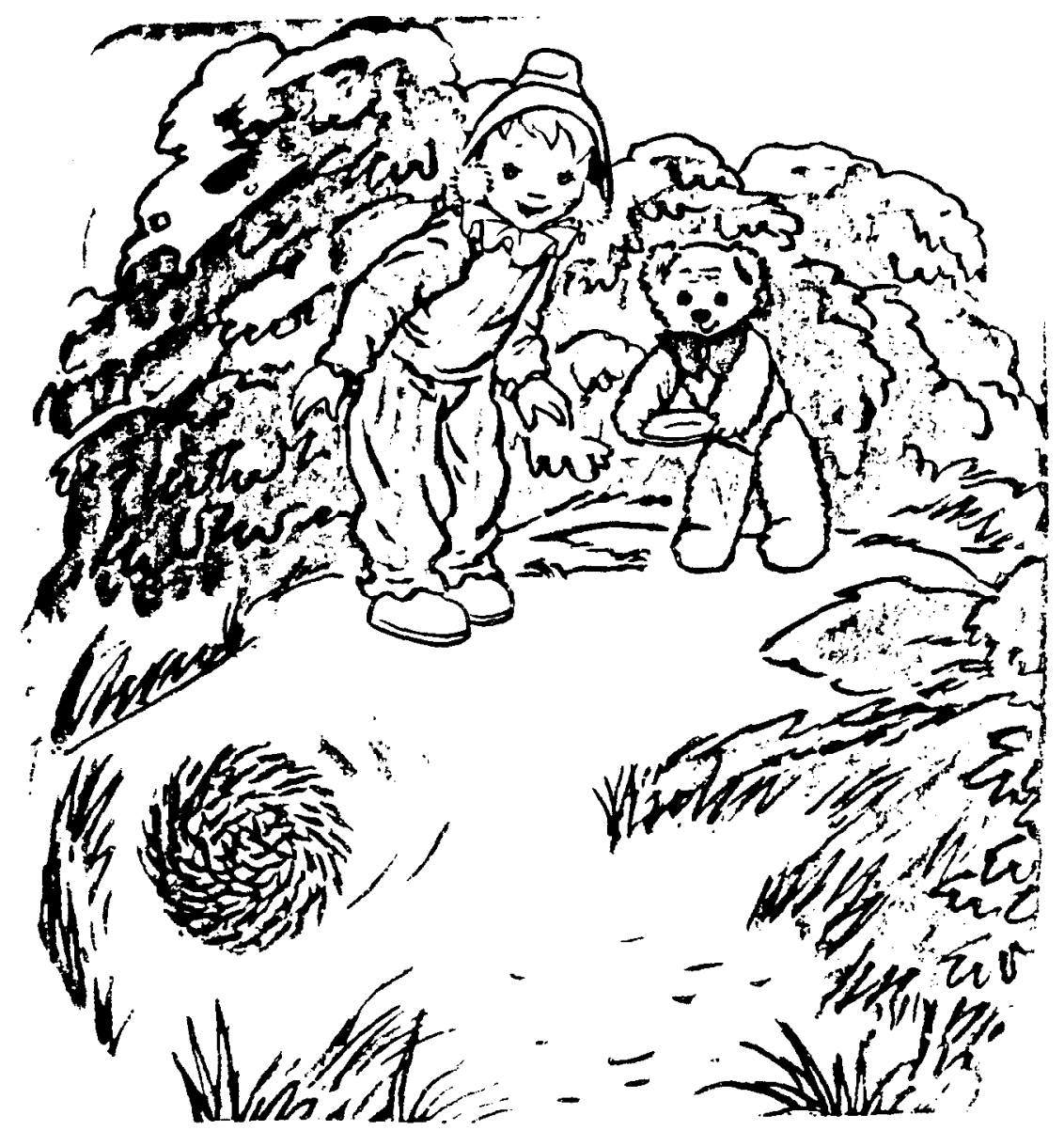

Fig. 28. A rotating hedgehog. 


\begin{abstract}
The $S$-matrix characterizing the scattering of pions from nucleons is calculated in the context of skyrmion models of the nucleon. These are models in which the nucleon is considered a soliton in the field of pions. The spectrum of nucleon and delta resonances in the Skyrme model is calculated and found o be in overall good agreement with Nature. Model-independent sum rules between amplitudes in the same partial wave are derived and examined. An extension of the formalism to the case of three light flavors is presented.
\end{abstract}




\section{ACKNOWLEDGMENTS}

I am indebted to my friend and collaborator Marek Karliner for hundreds of hours of vigorous discussions on all facets of this work. I feel especially privileged to have learned at the hands of a true keyboard virtuoso how useful a servant (and how jealous a mistress) a computer can be.

I have profited from discussions with many people including Larry Biedenharn, Yosef Dothan, Doron Gepner, Fred Gilman, Igor Klebanov, Rick Mackenzie, Mark Soldate, Marvin Weinstein and Shimon Yankielowicz. My understanding of the relevant experimental results has benefited from correspondence with Richard Arndt and G. Höhler, and especially from discussions with David Leith.

I would like to thank Eric D'Hoker for an elegant departmental colloquium on the topic of skyrmions which motivated me to drop whatever I was doing at the time in order to contribute to the renascence of this beautiful, if forgotten, subject.

Many thanks to Donald Knuth for assistance in typing.

I am indebted to my wife Judy and daughter Joanna for everything, most of all keeping me sane and making me happy through it all.

Finally, it has been a joy and privilege these last three years to have benefited from the gentle guidance of Michael Peskin: teacher and collaborator, babysitter and friend. I have come to share, not only his faith in the ultimate simplicity and elegance of Nature at its most basic level, but also-to my own surprisehis deep respect for experiment. The multitude of his flock is testimony to his extraordinary commitment to his students' education; if I am not the educated man I ought to be, it is not for his want of trying. Most of all, I will never forget, and can never hope to repay, his special dedication to his students on a personal level.

Thanks to all. 
To my dear parents, Daniel and Noémi, and in loving memory of my grandfather, Chaim Perelman. 


\section{TABLE OF CONTENTS}

FOREWORD 1

I. INTRODUCTION 5

1. Solitons in Effective Lagrangians 5

2. Large- $N$ QCD 14

II. SYSTEMATICS OF $\pi N$ SCATTERING IN CHIRAL SOLITON MODELS 24

1. Assumptions, Approximations, and Apologies 25

2. Derivation of Linear Relations 32

3. Comparison with Experiment: $\pi N \rightarrow \pi N \quad 36$

4. Comparison with Experiment: $\pi N \rightarrow \pi \Delta \quad 45$

III. THE BARYON SPECTRUM OF THE SKYRME MODEL 51

1. Pion-scattering from unrotated skyrmions 53

2. Pion-rucleon Scattering 60

IV. EXTENSION TO THREE FLAVORS 76

1. Formalism for 3-Flavor Scattering 76

2. The 3-Flavor Skyrme Model 82

3. Model-Independent Results 89

APPENDIX A: DERIVATION OF EQUATION (II.9) 96

APPENDIX B: EXPLICIT FORMULAE FOR $P$-SYMBOLS

APPENDIX C: FURTHER DETAILS ON SKYRME MODEL

EQUATIONS 105

APPENDIX D: EXPLICIT FORMULAE FOR $S U(3)$

SCATTERING COEFFICIENTS 109

REFERENCES 113 


\section{LIST OF FIGURES}

1. Graph of skyrmion profile. 12

2. Gluon correction to quark propagator. 15

3. Typical baryon self-interaction diagrams. $\quad 15$

4. Typical contribution to baryon-baryon scattering. 15

5. Typical contribution to meson-baryon scattering. 15

6. Relation of the six coupled angular momenta in either the initial or the final state of pion-skyrmion scattering. $\quad 31$

7. Experimental $I=\frac{3}{2} \pi N$ amplitudes compared with predicted linear combinations of $I=\frac{1}{2}$ amplitudes. $\quad 37-38$

8. Speed of motion of predicted $P_{33}$ amplitude. $\quad 40$

9. Motion of $\pi N$ amplitudes in the unitarity circle. 44

10. $\pi N \rightarrow \pi \Delta$ processes in which the pion jumps two units of angular momentum. $\quad 47$

11. $\pi N \rightarrow \pi \Delta$ processes in which intial and final pion angular momenta are equal. $\quad 48$

12. Comparison of $\pi N \rightarrow \pi N$ and $\pi N \rightarrow \pi \Delta$ in the same partial wave. 49

13. Phase shifts $\delta_{K K K}$ versus pion energy. $\quad 59$

14. Phase shifts $\delta_{K, K+1, K+1}$ versus pion energy. $\quad 59$

15. Phase shifts $\delta_{K, K-1, K-1}$ versus pion energy. $\quad 59$

16. Absorption parameters $\eta_{K}^{D}$ versus pion energy. $\quad 59$

17. Absorption parameters $\eta_{K}^{O D}$ versus pion energy. $\quad 59$

18. Phase shifts $\delta_{K, K-1, K+1}$ versus pion energy. $\quad 59$

19. Partial-wave $T$-matrix for the Skyrme model versus Nature. $62-66$

20. Possible movement due to $1 / N$ corrections of the poles and zeroes of the $S$-matrix, and the resulting effects on the amplitude near threshold. $\quad 68$

21. Phase shifts $\delta_{K L}^{k a 0 n}$ and $\delta_{K L}^{k-b a r}$ versus kaon momentum. 84

22. Comparison of 2- and 3-flavor Skyrme model amplitudes with experiment. 86 
23. The big-small-small-big pattern in both the 2- and 3-flavor Skyrme models. $\quad 87$

24. Speed of $T$-matrix for the four independent $F$-wave amplitudes in the 2- and 3-flavor Skyrme models. $\quad 87$

25. Elastic $K N$ amplitudes in the Skyrme model versus experiment.

26. Test of Eqs. (IV.14) and (IV.15). 95 


\section{FOREWORD}

This thesis is a compendium of my work on pion-nucleon scattering in the Skyrme model. ${ }^{[1-4]}$ This is a model in which the nucleon is considered, not as a bound state of three quarks, but rather as a soliton in the field of pions ${ }^{[5,6]}$ (henceforth "chiral soliton" or "skyrmion"). Note that we are thereby constructing fermions out of bose fields! Skyrme himself recognized the apparent difficulty of such a task:

This programme is the obverse of the more fashionable endeavour to reduce the truly elementary particles to a set of spinor fields, out of which everything can be built by simple conjunction. It is a priori much less reasonable because, in particular, it is more difficult to construct halfintegral representations of rotation groups out of integral than conversely; indeed it is patently impossible to do this within the limitations of a polynomial expansion. The hope that remains is that the particle-like states will be of a kind that cannot be reached by perturbation theory, and which cannot necessarily be discounted by general arguments. ${ }^{[6]}$

It was Finkelstein and Rubinstein who realized this hope by showing how a soliton could be endowed with fermi statistics. ${ }^{[7]}$ Later, Witten strengthened this result by proving the following remarkable theorem: ${ }^{[8]}$ the skyrmion must be quantized as a fermion if $N$, the number of colors of the underlying gauge theory (assuming that there is one), is odd, and as a boson if $N$ is even. This result (which, strictly speaking, holds only if the number of light flavors is greater than two) is consistent with the standard picture of a nucleon made out of $N$ quarks. Certainly it places Skyrme's bold identification of the nucleon as a soliton on a stronger theoretical footing.

The focus of our investigations will be effective Lagrangians of the form

$$
\mathcal{L}=\frac{f_{\pi}^{2}}{16} \operatorname{Tr}\left(\partial_{\mu} U \partial^{\mu} U^{\dagger}\right)+\cdots, \quad f_{\pi} \cong 186 \mathrm{MeV}
$$

where $U \in S U(2)$. The leading term is the usual 2-flavor nonlinear sigma model, while the dots stand for higher-derivative terms, which are not usually exploited 
in traditional soft-pion physics. The standard identification of the pion field in $(F .1)$ is via:

$$
U(x)=\exp \left(\frac{2 i}{f_{\pi}} \vec{\pi}(x) \cdot \vec{\sigma}\right)
$$

Thus the pions can be thought of as "small fluctuations" about the trivial vacuum $U(x) \equiv 1$.

The Lagrangian $(F .1)$ is invariant under the chiral transformations $S U(2)_{L} \times$ $S U(2)_{R}$ which take $U$ to $A U B^{-1}$. Note that the diagonal $S U(2)$ subgroup, with $B=A$, simply rotates $\vec{\pi}$ like a vector; this subgroup is isospin. In contrast, the "axial" transformations, with $B=A^{-1}$, act to first order like translations on the pion field. These are precisely the criteria necessary ${ }^{[0]}$ for identifying the pions with the pseudo-Goldstone bosons of $S U(2)_{L} \times S U(2)_{R} / S U(2)_{\text {isospin }}$, which describes the approximate flavor symmetries of the strong interactions.

It is a straightforward procedure to introduce additional fields to the model in such a way as to preserve chiral invariance. ${ }^{[0]}$ In particular, we can write down a Lagrangian describing the coupling of pions to the nucleon isodoublet $N$ :

$$
\mathcal{L}=\frac{f_{\pi}^{2}}{16} \operatorname{Tr}\left(\partial_{\mu} U \partial^{\mu} U^{\dagger}\right)+\bar{N}\left(i \gamma^{\mu} D_{\mu}-m\right) N+g_{A} D_{\mu} \vec{\pi} \cdot \bar{N} \vec{\tau} \gamma^{\mu} \gamma^{5} N
$$

Here $D$ is the covariant derivative appropriate to the nonlinear sigma model with variables in the manifold

$$
\frac{S U(2)_{L} \times S U(2)_{R}}{S U(2)_{\text {isospin }}}
$$

From this Lagrangian, all soft-pion theorems pertaining to the pion-nucleon interaction, such as Weinberg's calculation of the $S$-wave $\pi N$ scattering lengths, ${ }^{[10]}$ can be derived.

It is the moral of this thesis that, insofar as the $\pi N$ system is concerned, the Lagrangian (F.1) contains at least as much information as does ( $F .3)$ ! Not only does $(F .1)$ properly encompass soft-pion physics, as Schnitzer has shown, ${ }^{[1]}$ 
but in addition-well beyond the soft-pion regime-it yields surprisingly accurate predictions concerning the spectrum of nucleon and $\Delta$ resonances and the qualitative behavior of the large majority of $\pi N$ partial wave amplitudes.

The organization is as follows. In the first chapter, I shall motivate the Skyrme model from two seemingly orthogonal lines of thought. First, with the help of some elementary topology, I shall review how solitons can arise in a nonlinear field theory, and how they can be quantized as fermions. The second approach is that of large- $N$ QCD. The intent is to demonstrate that these approaches combine to form a pleasingly consistent picture of the nucleon. Apart from details of presentation, none of this work is my own.

Chapter II develops the group-theoretic formalism for $\pi N$ scattering for a broad class of models, Skyrme's included, in which the nucleon is constructed from a "hedgehog" soliton in the field of pions. Energy-independent linear relations between $\pi N \rightarrow \pi N$ and $\pi N \rightarrow \pi \Delta$ partial-wave amplitudes are derived from a leading-order large- $N$ analysis and shown to be in reasonable agreement with experiment. These relations can be considered model-independent tests of both the treatment of the nucleon as a soliton and the $1 / N$ expansion. It is also shown that the skyrmion approach provides a natural framework for understanding some striking qualitative features of the $\pi N$ partial-wave amplitudes. This work was done in collaboration with Michael Peskin. ${ }^{[2]}$

Chapter III, developed in collaboration with Marek Karliner, ${ }^{[1]}$ applies this formalism to the particular case of the Skyrme model. The spectrum of nucleon and $\Delta$ resonances of the model is calculated. The masses obtained are accurate on the average to $8 \%$ of their experimental values up to $3 \mathrm{GeV}$. For most partial waves, the model reproduces many significant features of the experimental Argand diagrams correctly, although there are severe discrepancies in some of the low partial waves.

Finally, Chapter IV presents an extension of the formalism corresponding to the case of three light quark flavors. ${ }^{|s|}$ This enables us to study the scattering 
of the pseudoscalar mesons from the baryon octet. It turns out that the modelindependent linear relations developed in Chapter II need to be modified, due to the presence in the 3 -flavor case of the Wess-Zumino term. The chapter concludes with results, recently obtained with Marek Karliner, ${ }^{[4]}$ for the 3-flavor Skyrme model. 


\section{INTRODUCTION}

\section{Solitons in Effective Lagrangians}

Skyrme recognized that the nonlinear nature of the Lagrangian $(F .1)$ permits a rich structure of field configurations that are topologically unconnected to the trivial vacuum. ${ }^{[s, 6]}$ To see this, consider the class of time-independent configurations $U(\bar{x})$. These define maps from $R^{3}$ into $S U(2)$. We will concentrate on the finite energy configurations. This condition requires $U(\vec{x}) \longrightarrow$ constant as $|\vec{x}| \longrightarrow \infty$. In turn, this allows us to adjoin the point at infinity to $R^{3}$, which yields a space that is topologically equivalent to the 3-sphere $S^{3}$. We are thus led to an examination of $\pi_{3}(S U(2))$, the group of homotopically distinct maps from the 3-sphere into the manifold $S U(2)$.

Now, elements of $S U(2)$ can be conveniently parametrized as

$$
a_{0} \cdot 1+i \vec{a} \cdot \vec{\sigma}
$$

where the $a_{\mu}$ 's are real numbers satisfying $a_{0}^{2}+|\vec{a}|^{2}=1$. So $S U(2)$ is also a 3sphere! Thus $\pi_{3}(S U(2)) \cong \pi_{3}\left(S^{3}\right)=Z$, the group of integers. In short, we have shown that the finite-energy static configurations $U(\vec{x})$ can be characterized by an integer, or winding number, which measures how many times the 3-sphere is "wrapped around" the group $S U(2)$. This number is topologically conserved, in the sense that two finite-energy configurations characterized by different winding numbers cannot be continuously deformed into one another without passing through configurations of infinite energy. Skyrme's bold proposal was the identification of this winding number with baryon number.

It is worth a brief digression to note that the existence of topologically stable nontrivial configurations is due to the nonlinear nature of $(F .1)$. This point becomes clear if we consider the "toy" example in $1+1$ dimensions of a single angular field $\theta(x, t)$, which takes values on $S^{1}$. Let us furthermore compactify 
the spatial variable $x$ into a circle. ${ }^{*}$ Since $\pi_{1}\left(S^{1}\right)=Z$, there are nontrivial configurations corresponding to a change of $\theta$ from (say) 0 to $2 \pi$ as $x$ likewise ranges from 0 to $2 \pi$. Now consider that, in addition to $\theta$, there is a radial field $\rho(x, t)$ in the picture; we also assume a standard "Mexican hat" potential with a circle of vacua at $\rho=\rho_{0}$. Then the nontrivial configuration described above is no longer topologically stable $\left(\pi_{1}\left(R^{2}\right)=1\right)$. To visualize this, imagine a rubber band wrapped tightly around the rim of the hat; it has winding number 1 and a finite energy (due to tension) which is analogous to the finite energy of the skyrmion (the minimum-energy configuration with baryon number 1 ). The rubber band has a non-zero probability of exploding up the cone of the hat, and landing flaccid at an arbitrary point in the rim; it is now in a "trivial vacuum," with zero energy, and has winding number zero. The moral for $3+1$ dimensions is clear: Had we started with a linear $\sigma$ model, characterized by the addition of a fourth field, the " $\sigma$ field", then our skyrmion would have been unstable against decay $\left(\pi_{3}\left(R^{4}\right)=1\right)$.

Returning to Eq. ( F.1), how do we actually construct a nontrivial finiteenergy static configuration? By chiral invariance, it suffices to consider the situation $U(\vec{x}) \longrightarrow 1$ as $|\vec{x}| \longrightarrow \infty$. Following Skyrme, we further restrict our attention to maximally symmetric "hedgehog" configurations

$$
U(\vec{x})=\exp (i F(r) \widehat{\mathbf{r}} \cdot \vec{\sigma})=\cos F(r) \cdot 1+i \sin F(r) \widehat{\mathbf{r}} \cdot \vec{\sigma}
$$

(We note, for future reference, that $U$ is annihilated by the vectorial sum $\vec{K}=$ $\vec{I}+\vec{J}$ of isospin and angular momentum; this turns out to be the linchpin around which all the results of Chapts. II-IV revolve!) Our boundary condition implies $F(r) \longrightarrow 2 n \pi$ as $r \longrightarrow \infty$; without loss of generality we choose $n=0$. Furthermore, continuity at the origin requires $F(0)=m \pi$. Although $U$ is then continuous, note that, for nonzero $m$, its "logarithm" $\vec{\pi}(x)$ has a monopole-like

* Unfortunately, the requirement of finite energy does not accomplish this for us in $1+1$ dimensions. 
singularity at the origin. We will focus on the choice $m=1$, with $F(r)$ monotonically decreasing. Comparing (I.1) with (I.2), it is clear that we have succeeded in constructing a bijective mapping from $S^{3}$ onto $S U(2)$, with winding number \pm 1 . Let us fix our orientation convention henceforth so that this configuration is assigned a winding number of +1 . We have a baryon! It ought to be emphasized that any configuration of winding number one is homotopic to the hedgehog configuration we have constructed.

As a quantitative measure of baryon number density, consider the quantity

$$
\rho_{B}(x)=\frac{-1}{24 \pi^{2}} \epsilon_{i j k} \operatorname{Tr} T_{i} T_{j} T_{k}, \quad T_{i} \equiv U^{\dagger}(x) \partial_{i} U(x) .
$$

$\rho_{B}$ can be thought of as the time-component of the baryon current

$$
B^{\mu}=\frac{-1}{24 \pi^{2}} \epsilon^{\mu \nu \alpha \beta} \operatorname{Tr} T_{\nu} T_{\alpha} T_{\beta}
$$

This quantity has the following desirable properties:

1) It is automatically conserved:

$$
\partial_{\mu} B^{\mu}=0
$$

This follows from the identity

$$
\partial_{\mu} T_{\nu}=-T_{\mu} T_{\nu}+U^{\dagger} \partial_{\mu} \partial_{\nu} U
$$

and the cyclic invariance of the trace.

2) For the hedgehog configuration (I.2),

$$
\rho_{B}(\vec{x})=-\frac{1}{2 \pi^{2} r^{2}} F^{\prime} \sin ^{2} F=-\frac{1}{4 \pi^{2} r^{2}} \frac{d}{d r}\left(F-\frac{1}{2} \sin 2 F\right) .
$$

Integrating over space yields a baryon number $B=F(0) / \pi=+1$ as desired. 
3) Baryon number so defined is invariant under deformations; this prevents a baryon from decaying by emitting pions. A small perturbation on a configuration can be parametrized by $U \longrightarrow U W$ with $W=1+i \vec{\epsilon} \cdot \vec{\sigma}+O\left(\epsilon^{2}\right)$. This change induces

$$
T_{\mu} \longrightarrow W^{\dagger}\left(T_{\mu}+\widetilde{T}_{\mu}\right) W, \quad \widetilde{T}_{\mu}=\partial_{\mu} W \cdot W^{\dagger}=i \partial_{\mu} \vec{\epsilon} \cdot \vec{\sigma}+O\left(\epsilon^{2}\right)
$$

Thus

$$
\rho_{B} \longrightarrow \frac{-1}{24 \pi^{2}} \epsilon_{i j k} \operatorname{Tr}\left(T_{i}+\tilde{T}_{i}\right)\left(T_{j}+\widetilde{T}_{j}\right)\left(T_{k}+\widetilde{T}_{k}\right)=\rho_{B}-\frac{i}{8 \pi^{2}} \epsilon_{i j k} \operatorname{Tr}\left(\partial_{i} \vec{\epsilon} \cdot \vec{\sigma} T_{j} T_{k}\right)+O\left(\epsilon^{2}\right) .
$$

Dropping the $O\left(\epsilon^{2}\right)$ contribution and using (I.6), we can rewrite this as

$$
\Delta \rho_{B}=\frac{-i}{8 \pi^{2}} \epsilon_{i j k} \partial_{i} \operatorname{Tr}\left(\vec{\epsilon} \cdot \vec{\sigma} T_{j} T_{k}\right)
$$

which is a total derivative, vanishing upon integration. This proves the claim.

4) The baryon number of any configuration that can be continuously deformed to the trivial vacuum $U \equiv 1$ is zero. This follows immediately from the previous observation. In particular, configurations built from the usual pion fields as given in ( $F .2)$, which are continuous, singularity-free and vanish at infinity, are purely "mesonic," with $B=0$.

5) The baryon number of a product $U_{1} U_{2}$ is just the sum of the individual baryon numbers:

$$
\int d^{3} x \rho_{B}\left(U_{1} U_{2}\right)=\int d^{3} x\left(\rho_{B}\left(U_{1}\right)+\rho_{B}\left(U_{2}\right)\right)
$$

To see this, note that

$$
\rho_{B}\left(U_{1} U_{2}\right)=\frac{-1}{24 \pi^{2}} \epsilon_{i j k} \operatorname{Tr}\left(T_{i}+\tilde{T}_{i}\right)\left(T_{j}+\tilde{T}_{j}\right)\left(T_{k}+\tilde{T}_{k}\right), \quad T_{i}=U_{1}^{\dagger} \partial_{i} U_{1}, \quad \tilde{T}_{i}=\partial_{i} U_{2} \cdot U_{2}^{\dagger}
$$


This can be written

$$
\rho_{B}\left(U_{1} U_{2}\right)=\rho_{B}\left(U_{1}\right)+\rho_{B}\left(U_{2}\right)-\frac{1}{8 \pi^{2}} \epsilon_{i j k} \operatorname{Tr} T_{i}\left(T_{j}+\tilde{T}_{j}\right) \tilde{T}_{k}
$$

But the last term can be expressed as a total derivative

$$
\frac{-1}{8 \pi^{2}} \epsilon_{i j k} \partial_{j} \operatorname{Tr} T_{i} \tilde{T}_{k}
$$

which follows from (I.6) together with the identity

$$
\partial_{j} \widetilde{T}_{k}=-\widetilde{T}_{k} \widetilde{T}_{j}+\partial_{j} \partial_{k} U_{2} \cdot U_{2}^{\dagger}
$$

This establishes the claim.

6) The hermitian conjugate $U^{\dagger}$ of a baryon-number-one configuration has baryon number -1 ; this is an immediate consequence of the previous claim. So the baryons have antiparticles.

7) The superposition of two pion field configurations of baryon number one is a configuration of baryon number two. To see this, consider deforming each configuration to a tightly localized hedgehog and then separating the centers sufficiently so that the regions of nonzero field do not overlap. Then $U$ factors into a product $U_{1} U_{2}$, and the claim is established. By similar arguments it follows that the baryon number of a superposition of $n$ configurations of baryon number 1 and $m$ configurations of baryon number -1 is just $n-m$.

8) Finally, we would like to be able to say something about the location of the baryon. ${ }^{[6]}$ In particular, for a field configuration of the type (I.2), our intuition tells us that the baryon is concentrated about the center of the hedgehog, where $U=-1$. Typically $\rho_{B}$ will be maximized here. In addition, it is suggestive that 
the "velocity" of this particle is proportional to the baryon number flux, defined in Eq. (I.4). To see this, let us assume that $U\left(x_{0}, t_{0}\right)=U\left(x_{0}+\delta x, t_{0}+\delta t\right)=-1$. This implies that $v^{\mu} \partial_{\mu} U=0$. Multiplying by $U^{\dagger}$ and tentatively equating $v^{\mu}$ with $B^{\mu}$, this becomes $B^{\mu} T_{\mu}=0$. To verify immediately that this is so, it suffices to rewrite $T_{\mu}$ as $\frac{1}{2} \sum_{l} \sigma^{l} \operatorname{Tr}\left(\sigma^{l} T_{\mu}\right)$ and $B^{\mu}$ as $\frac{-i}{96 \pi^{2}} \epsilon^{\mu \nu \alpha \beta} \epsilon_{i j k} \operatorname{Tr}\left(\sigma^{i} T_{\nu}\right) \operatorname{Tr}\left(\sigma^{j} T_{\alpha}\right) \operatorname{Tr}\left(\sigma^{k} T_{\beta}\right)$. Since the index $l$ must equal one of the indices $i, j, k$, the product vanishes by antisymmetry. We have established $v_{\mu} \propto B_{\mu}$.

Thus far we have succeeded in concocting a plausible facsimile of a baryon. In addition, we have constructed a quantity that has many of the preperties one would desire from a baryon number current. However, we have not yet come up with a nucleon. Such an object ought to be identified with a minimum energy configuration in the $B=1$ sector. More importantly, it should have spin and isospin $\frac{1}{2}$. Let us deal with these two considerations in turn.

It is easy to show that the leading term in the Lagrangian ( $F .1)$ is not in itself able to support a soliton, i.e., a nontrivial stable static classical solution. To see this, assume the existence of such a soliton $U_{0}(\vec{x})$. Its mass (i.e., energy in its rest frame) would be given by

$$
E_{0}=\frac{f_{\pi}^{2}}{16} \int d^{3} x \operatorname{Tr} \partial_{i} U_{0}^{\dagger}(\bar{x}) \partial_{i} U_{0}(\bar{x})
$$

Now consider the family of configurations $U_{0}(\vec{x} ; \lambda)=U_{0}(\lambda \vec{x})$; these have $E_{0}(\lambda)=$ $\lambda^{-1} \cdot E_{0}$. This is minimized, not for $\lambda=1$ as we assumed, but rather for $\lambda \longrightarrow \infty$. Put another way, the soliton shrinks to zero size. This is, of course, Derrick's Theorem. ${ }^{[12]}$

The situation is not improved by adding a chiral symmetry-breaking mass term $\operatorname{Tr}\left(U M+M^{\dagger} U^{\dagger}\right)$ to the Lagrangian; such a term would give a contribution to the energy that scaled like $\lambda^{-3}$, and the soliton would still shrink away. Instead, it is necessary to add a term with four or more derivatives. Skyrme proposed the 
Lagrangian

$$
\mathcal{L}=\frac{f_{\pi}^{2}}{16} \operatorname{Tr} \partial_{\mu} U \partial^{\mu} U^{\dagger}+\frac{1}{32 e^{2}} \operatorname{Tr}\left[\left(\partial_{\mu} U\right) U^{\dagger},\left(\partial_{\nu} U\right) U^{\dagger}\right]^{2}
$$

The second term, or "Skyrme term," is the unique chiral- and Lorentz-invariant four-derivative term containing no more than two time derivatives; it is thus amenable to a standard Hamiltonian treatment. For our present purposes, what is important is that its contribution to the energy scales like $\lambda$. Thus it permits a stable classical configuration of nonzero extent, for which the energy contributions of the first and second terms in (I.17) will be precisely equal.

The Euler-Lagrange equation obtained from (I.17) is extremely complicated. Skyrme's simplifying ansatz was that the minimum-energy solutions of nonzero baryon number could be written in the hedgehog form (I.2). The resulting equation for $F(r)$ is reproduced as Eq. (III.4) below; the solution with boundary conditions appropriate for $B=1$ is graphed in Fig. 1 (drawn from Ref. 13). Although it follows from the numerical analysis presented in Chapter III that this configuration is locally stable, the question of whether it is in fact a global minimum in the $B=1$ sector has never been fully established. Nevertheless, this is universally believed, and we will assume in what follows that it is.

The second crucial link in the identification of the skyrmion with the nucleon is the problem of endowing the soliton with the quantum numbers $I=J=\frac{1}{2}$. The possibility of quantizing a soliton as a fermion was established long ago by Finkelstein and Rubinstein. ${ }^{[7]}$ We will now review an explicit construction given by Adkins, Nappi and Witten. ${ }^{[13]}$

This construction begins with the realization that a hedgehog soliton $U_{0}$ picks out only one of an infinite class of possible orientations between spin and isospin axes; equally acceptable solutions to $(F .1)$ are furnished by

$$
U_{A} \equiv A U_{0} A^{-1} \equiv e^{i F(r)\left(D^{1}(A) \widehat{r}\right) \cdot \vec{\sigma}}
$$

where $A$ is any (constant) $S U(2)$ group element with spin-1 representation $D^{1}(A)$. 


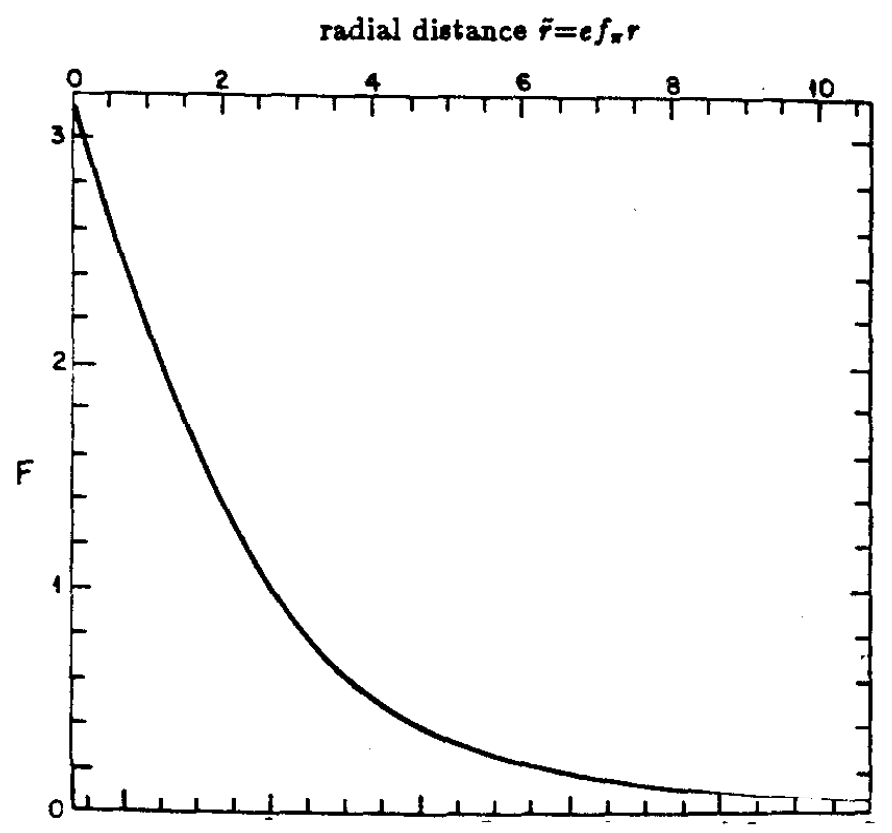

Fig. 1. Graph of skyrmion profile

The natural action of isospin and angular momentum on this rotated soliton is given by:

$$
\text { Isospin : } \quad U_{A} \rightarrow U_{I} A U_{0} A^{-1} U_{I}^{-1}=U_{\left(U_{I} A\right)}
$$$$
\text { Angular Momentum: } \quad U_{A} \rightarrow A U_{J}^{-1} U_{0} U_{J} A^{-1}=U_{\left(A U_{J}^{-1}\right)}
$$

It is fruitful at this point to think of $A$, the so-called "rotational collective coordinate" of the soliton, as a quantum-mechanical variable that takes values on the group manifold $S U(2)$. We will let $\left|U_{A}\right\rangle$ denote the state containing a chiral soliton in the orientation $A$; it satisfies

$$
<U_{A^{\prime}} \mid U_{A}>=\delta\left(A-A^{\prime}\right)
$$

A suitable candidate for a physical baryon is then a coherent superposition

$$
|\Psi\rangle=\int d A \chi(A)\left|U_{A}\right\rangle
$$

Here $d A$ represents the group-invariant measure, normalized so that $\int d A=$ $2 \pi^{2}$, and the wavefunction $\chi(A)$ is chosen appropriately to make this expression 
an eigenstate of both isospin $\vec{i}$ and spin $\vec{s}$. One may think of this expression as describing a rotating soliton. Under the simultaneous action of isospin and angular momentum,

$$
|\Psi\rangle \longrightarrow \int d A \chi(A)\left|U_{\left(U_{I} A U_{J}^{-1}\right)}\right\rangle=\int d A \chi\left(U_{I}^{-1} A U_{J}\right)\left|U_{A}\right\rangle
$$

In other words, the transformations induce a change

$$
\chi(A) \longrightarrow \chi\left(U_{I}^{-1} A U_{J}\right)
$$

in the soliton's wavefunction.

The construction of the wavefunctions $\chi(A)$ corresponding to definite states of $\left(i, i_{z}\right)$ and $\left(s, s_{z}\right)$ is now an easy exercise. ${ }^{[13]}$ In fact (for two flavors) one has the choice of quantizing the collective coordinates either as fermions or as bosons. For nucleons (with $i=s=\frac{1}{2}$ ) one finds

$$
\chi_{i_{x} s_{x}}^{\left(\frac{1}{2}\right)}(A)=\frac{i}{\pi}\left(\epsilon A^{\dagger}\right)_{s_{x} i_{z}} ; \quad \epsilon=\left(\begin{array}{cc}
0 & 1 \\
-1 & 0
\end{array}\right) .
$$

Indeed, this transforms as

$$
\chi \rightarrow \frac{i}{\pi}\left(\epsilon U_{J}^{\dagger} A^{\dagger} U_{I}\right)_{8 i_{z}}=\frac{i}{\pi}\left(U_{J}^{T} \epsilon A^{\dagger} U_{I}\right)_{s_{x} i_{z}}
$$

which is just what one wants. Likewise, a general rotational eigenstate, with $i=s=R$, has as its properly normalized wavefunction ${ }^{\star}$ :

$$
\chi_{i_{x} \delta_{x}}^{R}(A)=\frac{i}{\pi} \sqrt{\frac{1}{2}(2 R+1)}\left(\epsilon^{R} D^{R}(A)^{\dagger}\right)_{B_{x} i_{z}}
$$

Since the $\chi$ 's form a complete set of functions on $S U(2)$, the result $i=s$ holds in general. Those states with $i=s \geq \frac{5}{2}$ are unphysical artifacts of the model,

* Of course, for each $R$, we have the freedom to redefine the wavefunctions $\chi_{i_{z}, z}^{R}$ by a common phase; our choice agrees with Ref. 13 for the nucleons but differs by a sign for the deltas, in order to conform in this case to Ref. 14. A different phase convention modifies the linear relation (II.21) below. 
although they would be present in a world with more than three colors. The key result obtained from quantizing the collective coordinates is that the quantization energy is proportional to $i(i+1) .^{[13]}$ Thus, in these models, the lowest-lying fermionic states in the $B=1$ sector of the theory correctly correspond to the nucleon, while the first excited states have the quantum numbers of the $\Delta$. We will see in Chapters II and III that states with $i \neq s$, corresponding to the observed nucleon and $\Delta$ resonances, emerge naturally from a consideration of pion-skyrmion scattering.

Equations (I.20), (I.21) and (I.26) allow one to calculate expectation values of various currents between nucleon states. As a result, a host of static properties of the nucleon and $\Delta$, such as magnetic moments and charge radii, can be obtained. ${ }^{[13]}$ It is the semiquantitative success of these calculations $(20-30 \%$ typically) that is largely responsible for the resurgence of interest in the Skyrme model after a nearly twenty year hibernation.

\section{Large- $N$ QCD}

We will now discuss another approach to the depiction of baryons as solitons, that of large- $N$ QCD. This is the scheme in which the gauge group $S U(3)_{\text {color }}$ of the strong interactions is replaced by $S U(N)$, with $N$ taken to be large. The qualitative phenomenological successes of large- $N$ in describing meson physics are well-known. ${ }^{[15]}$ Mesons are stable in this limit, with decay rates and twobody scattering amplitudes vanishing like $1 / \sqrt{N}$ and $1 / N$, respectively. They have masses of order $N^{0}$, as they should, since regardless of $N$ they are always composed of one quark and one antiquark. (Admixtures of $q \bar{q} q \bar{q}$ etc., already suppressed experimentally when $N=3$, become more and more negligible as $N$ gets large.) As $N \longrightarrow \infty$, phenomenological rules of thumb such as Zweig's rule and the dominance of 2-body decays become exact; indeed, the very existence of mesons in each $J^{P C}$ channel allowed by the quark model is guaranteed. We will now review what large- $N$ has to say about baryons. This approach was developed by Witten, ${ }^{[16]}$ whom we shall follow closely. 


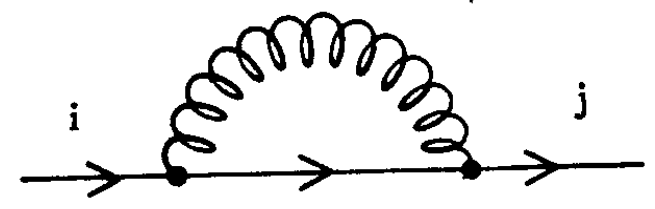

Fig. 2. Gluon correction to quark propagator

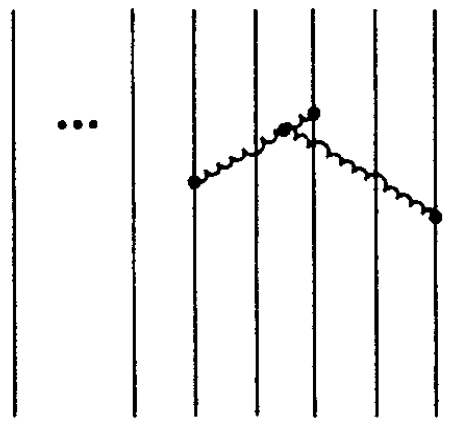

(a)

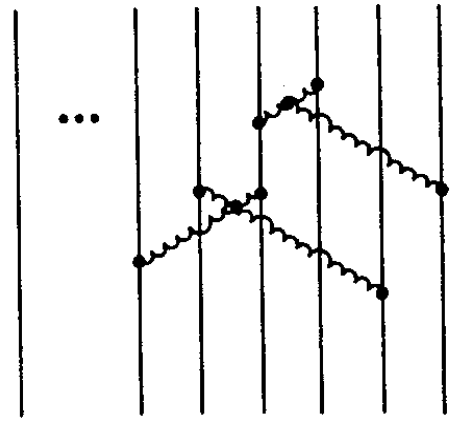

(b)

Fig. 3. Typical baryon eelf-interaction diagrams
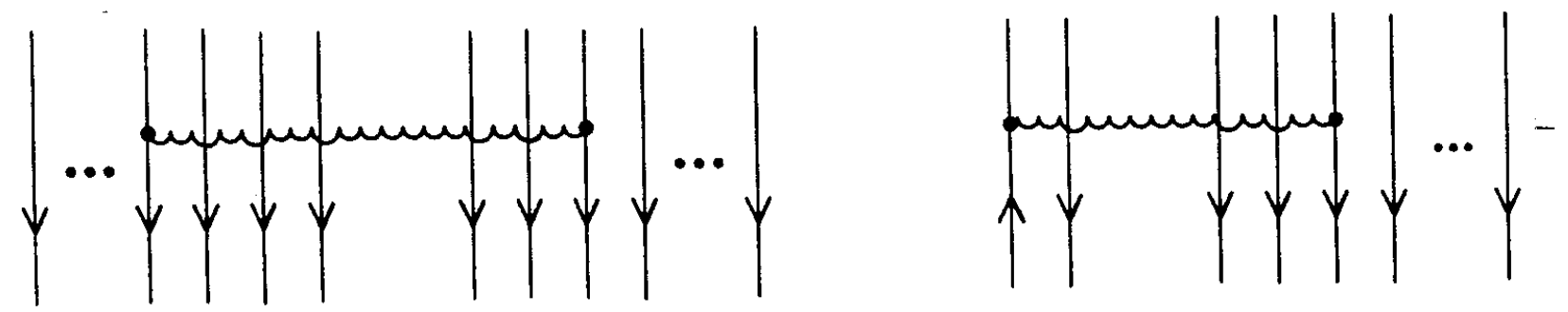

Fig. 4. Typical contribution to baryon-baryon scattering

Fig. 5. Typical contribution to meson-baryon acattering 
In large- $N$, there are $N$ quark degrees of freedom but $N^{2}-1 \approx N^{2}$ gluonic degrees of freedom. Consider the simple gluonic correction to the quark propagator depicted in Fig. 2. Even after we specify the color index of the external quark, this diagram receives a combinatoric factor of $N$ corresponding to the $N$ possible values for the index of the internal quark. Thus, if we want the theory to have a smooth-but nontrivial-limit as $N \longrightarrow \infty$, we must require that the $\bar{q} q g$ vertex scale like $1 / \sqrt{N}$. By similar arguments, the 3- and 4-gluon vertices must scale like $1 / \sqrt{N}$ and $1 / N$, respectively.

With this knowledge, it is easy to see that the mass of a baryon is of order $N$, as one naively expects for an object built from $N$ quarks. Figure 3 shows two typical connected diagrams which contribute to the energy of a baryon. Figure $3 a$ should be multiplied by a combinatoric factor proportional to $N^{3}$, which comes from choosing the three quark lines that couple to gluons. In addition, it has four $\bar{q} q g$ or $g g g$ vertices. Thus it scales like $N$, as asserted. Figure $3 b$ has a combinatoric factor of $N^{6}$ and a suppression of $N^{-5}$ from the vertices, so it too is proportional to $N$.

By the same naive methods we can arrive at the correct $N$-dependence of a variety of scattering processes. For example, Fig. 4 shows a typical contribution to baryon-baryon scattering. It is easy to verify that this diagram contributes a factor of $N^{2} /(\sqrt{N})^{2}=N$. Thus the interaction terms in baryon-baryon scattering are of the same order of magnitude as the kinetic energy and mass terms of the two-baryon system, and baryon-baryon scattering has a smooth, nontrivial large$N$ limit. The same holds for baryon-antibaryon scattering.

Figure 5 displays a typical graph contributing to meson-baryon scattering, which is the topic of this thesis. It is clear that this diagram is of order 1. Thus, in large- $N$, the baryon doesn't "feel" the meson at all, while the meson, whose free Hamiltonian is itself of order 1, scatters in a nontrivial way from the baryon. This jibes with our intuition of a baryon as a heavy object in large- $N$ which would be unperturbed by the influence of a meson, which is just a $\bar{q} q$ boundstate; the 
meson, in contrast, sees the baryon as a fixed source and scatters accordingly.

We can gain additional insight by considering the idealized example of $N$ very heavy quarks. Since, in a baryon, the quarks are already antisymmetric in color indices, they must necessarily be symmetric under the simultaneous exchange of spin, isospin and spatial wavefunctions. Following Witten, we will restrict ourselves to the particularly simple case in which they have been placed in identical states of spin and isospin. The $N$-quark state must then be symmetric under exchange of spatial wavefunctions; we have, in effect, a system of bosons.

Now, for any $N$, a heavy quark system can reasonably be modeled by a nonrelativistic Schrödinger equation:

$$
H=N m+\sum_{i=1}^{N} \frac{-\nabla_{i}^{2}}{2 m}+\frac{\alpha_{2}}{N} \sum_{i<j} V_{2}\left(x_{i}, x_{j}\right)+\frac{\alpha_{3}}{N^{2}} \sum_{i<j<k} V_{3}\left(x_{i}, x_{j}, x_{k}\right)+\cdots
$$

Here $V_{2}$ is the ordinary 2-body force, $V_{3}$ is the three-body force resulting from diagrams such as Fig. $3 a$, etc., while the $\alpha_{i}$ 's are coupling constants which scale like $N^{0}$. The $N$ dependence of the various terms follows from the naive diagrammatic analysis presented above.

The crucial point is the following. As $N$ gets very large, each quark feels, in effect, the same average potential obtained from summing the $O(1 / N)$ contributions from each of the other $N-1$ quarks, the $O\left(1 / N^{2}\right)$ contributions from each of the other $(N-1)(N-2) / 2$ quark pairs, etc. Fluctuations about this mean-field potential become increasingly negligible as $N \rightarrow \infty$. Thus in this limit the $N$-quark spatial wavefunction $\Psi\left(x_{1}, \ldots, x_{N}\right)$ describing the ground state of the system should be written simply as a product

$$
\Psi\left(x_{1}, \ldots, x_{N}\right)=\prod_{i=1}^{N} \phi\left(x_{i}\right)
$$

of $N$ identical one-particle wavefunctions, each of which is in the ground state of the mean potential; this is the Hartree approximation. 
Inserting this product ansatz into the Hamiltonian yields

$$
\begin{aligned}
& <\Psi|H| \Psi>=N m-N \int d^{3} x \frac{1}{2 m} \phi^{*} \nabla^{2} \phi \\
& +\frac{1}{2} N^{2}\left(\frac{\alpha_{2}}{N}\right) \int d^{3} x \int d^{3} y \phi^{*}(x) \phi(x) \phi^{*}(y) \phi(y) V_{2}(x, y) \\
& +\frac{1}{3 !} N^{3}\left(\frac{\alpha_{3}}{N^{2}}\right) \int d^{3} x \int d^{3} y \int d^{3} z \phi^{*}(x) \phi(x) \phi^{*}(y) \phi(y) \phi^{*}(z) \phi(z) V_{3}(x, y, z) \\
& +\cdots+O\left(N^{0}\right)
\end{aligned}
$$

The resulting eigenstate equation for $\phi$ is found by varying with respect to $\phi^{*}(x)$ :

$$
\begin{aligned}
& -\frac{1}{2 m} \nabla^{2} \phi(x)+\frac{1}{2} \alpha_{2} \phi(x) \int d^{3} y \phi^{*}(y) \phi(y) V_{2}(x, y) \\
& +\frac{1}{3 !} \alpha_{3} \phi(x) \int d^{3} y \int d^{3} z \phi^{*}(y) \phi(y) \phi^{*}(z) \phi(z) V_{3}(x, y, z)+\cdots=\epsilon_{0} \phi(x)
\end{aligned}
$$

with $\epsilon_{0}$ the average quark ground state energy. This self-consistent equation can be solved, in principle, by iteration, with the simplifying radial ansatz $\phi(x)=$ $\phi(|x|)$ which is surely true for the ground state wavefunction. For our purposes, the important feature of (I.30) is the simple observation that $N$ has factored smoothly out of this equation, implying that the size and shape of the baryon are quantities of $O\left(N^{0}\right)$, in contrast to its mass.

With this back-of-the-envelope apparatus we can "calculate" various scattering processes as well. For example, baryon-antibaryon scattering can be described by a wavefunction

$$
\Psi\left(x_{1}, \ldots, x_{N}, y_{1}, \ldots, y_{N}\right)=\prod_{i=1}^{N} \phi\left(x_{i}\right) \prod_{j=1}^{N} \omega\left(y_{j}\right)
$$

which results in two coupled equations similar to (I.30) for $\phi$ and $\omega, N$ factoring out as before; likewise for baryon-baryon scattering. 
Meson-baryon scattering can be described by introducing a meson wavefunction $u(x, y)$, with $x$ the quark position and $y$ the antiquark position. Let us place the quark in the same spin-isospin state as the quarks of the baryon. Then, since these exhaust the available color degrees of freedom, the meson quark must be orthogonalized in position space:

$$
\int d x \phi^{*}(x) u(x, y)=0
$$

We consider the meson-baryon wavefunction:

$$
\Psi_{M B}\left(x_{1}, \ldots, x_{N+1}, y\right)=\text { const. } \quad \times \quad \sum(-1)^{\pi} \prod_{i=1}^{N} \phi\left(x_{\pi(i)}\right) u\left(x_{\pi(N+1)}, y\right)
$$

where we have antisymmetrized the meson's quark with respect to the others by summing over permutations as indicated. (Color indices are suppressed as always.) Inserting $\Psi_{M B}$ into the Hamiltonian (I.27) yields

$$
\begin{aligned}
& <\Psi_{M B}|H| \Psi_{M B}>=(N+2) m-N \int d^{3} x \frac{1}{2 m} \phi^{*} \nabla^{2} \phi \\
& +\frac{1}{2} N^{2}\left(\frac{\alpha_{2}}{N}\right) \int d^{3} x \int d^{3} y \phi^{*}(x) \phi(x) \phi^{*}(y) \phi(y) V_{2}(x, y) \\
& -\int d^{3} x \int d^{3} y \frac{1}{2 m} u^{*}(x, y)\left(\nabla_{x}^{2}+\nabla_{y}^{2}\right) u(x, y) \\
& +\frac{\alpha_{2}}{N} \int d^{3} x \int d^{3} y u^{*}(x, y) u(x, y) V_{2}(x, y) \\
& +\frac{\alpha_{2}}{N} \cdot N \int d^{3} x \int d^{3} y \int d^{3} z \phi^{*}(z) \phi(x) u^{*}(x, y) u(z, y)\left[V_{2}(x, z)+V_{2}(y, z)\right]+\cdots
\end{aligned}
$$

Here we have dropped the 3-body terms, and assumed the same force for $q q, \bar{q} q$ and $\bar{q} \bar{q}$.

From (1.34) one obtains the Schrödinger equations for the baryon and meson by varying with respect to $\phi^{*}(x)$ and $u^{*}(x, y)$, respectively. The key point is 
the following. It is clear that for the baryon equation of motion, the leading terms, which are $O(N)$, are completely independent of the meson field $u(x, y)$. In fact, we obtain just the "unperturbed" equation as given by (I.30). In contrast, for the meson, the last line of (I.34) gives a nontrivial baryonic contribution to the equations of motion. We have confirmed the naive picture given earlier, according to which, for large $N$, the baryon is impervious to the meson, while the meson scatters nontrivially from the baryon. What we have discovered is that the leading-order equation of motion for the meson is a linear one.

At this point, we should pause to compare the qualitative picture of the large$N$ baryon with that obtained in the previous section. $N$ enters the Lagrangian (F.1) through $f_{\pi}$, which $\sim \sqrt{N}$. We make the following points:

1) The size and shape of the baryon in both pictures is independent of $N$ as $N \rightarrow \infty$. Indeed, from the chiral soliton point of view, the shape of the skyrmion emerges from the classical equations of motion obtained from $(F .1)$,

$$
\partial_{i}\left(U^{\dagger} \partial_{i} U\right)+\cdots=0
$$

which is manifestly $N$-independent, since $f_{\pi}$ has been factored out. In the quark picture, the baryon is given as the self-consistent solution to an $N$-independent Hartree equation as typified by (I.30). Note that both kinds of equation are completely nonlinear.

2) In contrast, the mass of the baryon is proportional to $N$. We have confirmed this in the quark picture by considering typical connected diagrams such as Fig. 3. In the soliton picture, the same conclusion emerges from Eq. (I.16).

3) Baryon-baryon and baryon-antibaryon scattering in both pictures is determined by complicated coupled nonlinear equations. We have convinced ourselves of this in the quark picture. In a soliton approach, one starts by considering initial conditions in which the two solitons (or the soliton and antisoliton) are widely separated, and evolves them toward one another via Eq. (I.35). As is well 
known, this is, in general, a highly nonlinear procedure which poses an enormous numerical challenge, even in $1+1$ dimensions.

4) In contrast, meson-baryon scattering, to leading order in $1 / N$, is characterized by linear equations of motion for the meson in the baryon background. We have seen this explicitly in the context of the "heavy quark" model considered above. This characteristic is implicit in the soliton approach as well, as discussed in Section II.1.

The two seemingly orthogonal approaches to the baryon which we have considered have converged into a remarkably self-consistent picture! It is known from 't Hooft's analysis that, as $N \rightarrow \infty$, QCD becomes better and better described as a theory, not of quarks and gluons, but of hadrons: mesons and glueballs. ${ }^{[15]}$ Accordingly, $\mathcal{L}_{Q C D}$ can be replaced in this limit by an effective meson Lagrangian with an infinite number of fields, to which the Skyrme Lagrangian (I.17) can be thought of as a low-energy approximation. What we have discovered is that, on the one hand, baryons in large- $N$ have many of the properties one usually associates with solitons, and on the other hand, that effective Lagrangians such as (I.17) do in fact possess soliton solutions whose lowest-lying fermionic excitations have $I=J=\left\{\frac{1}{2}, \frac{3}{2}\right\}$ : precisely the quantum numbers of the nucleon and $\Delta$.

Another connection between the two approaches is due to Manohar, ${ }^{[17]}$ who showed that all purely group-theoretic results (e.g., $F / D$ ratios) obtained in the chiral soliton model are exactly the same as one would obtain in the naive large- $N$ quark model. The essence of his argument is easy to give for the case of two flavors. Consider the matrix element between initial and final soliton states, as given by Eqs. (I.21) and (I.26), of an irreducible tensor operator $\hat{0}$ which transforms like $\mid J, J_{z}>$ and $\mid I, I_{z}>$ under angular momentum and isospin, respectively. By the Wigner-Eckart theorem, the matrix element

$$
\left.<\Psi^{\prime}|\hat{O}| \Psi\right\rangle \equiv \int d A \chi_{i_{s}^{\prime} s_{s}^{\prime}}^{R^{\prime} \dagger}(A) \hat{O}(A) \chi_{i_{s} s_{s}}^{R}(A)
$$


is proportional to the product of Clebsch-Gordan coefficients

$$
<R^{\prime} i_{z}^{\prime}\left|I R I_{z} i_{z}><R^{\prime} s_{z}^{\prime}\right| J R J_{z} s_{z}>
$$

where the constant of proportionality, the "reduced matrix element," contains the relevant dynamical information about $\hat{O}$ but is independent of $I_{z}$ and $J_{z}$. By a "purely group-theoretic result" we mean one that does not depend on the details of this reduced matrix element, but only on the structure of (I.37).

We would now like to repeat this little exercise in the naive large- $N$ quark model. Following Manohar, let us consider the $N$-quark state

$$
\Psi_{0}=2^{-N / 2} \cdot|u \downarrow-d \uparrow>| u \downarrow-d \uparrow>\mid u \downarrow-d \uparrow>\cdots
$$

This state is annihilated by $\vec{I}+\vec{J}$, so it is in fact the proper quark-model analog of the hedgehog configuration (I.2)! As in Eq. (I.18), we can likewise construct the states $\Psi_{A}$ in which the isodoublet $\left(\frac{u}{d}\right)$ is rotated by an $S U(2)$ matrix $A$ relative to the spin doublet. Then, just as in the chiral soliton case, we can form "large- $N$ " nucleons and $\Delta$ 's by considering a linear superposition of the $\Psi_{A}$ 's, weighted by the very same wavefunctions $\chi(A)$ defined in (I.26). The matrix element of the quark-model analog of $\hat{O}$ is then given by:

$$
\left\langle\Psi^{\prime}|\hat{O}| \Psi\right\rangle=\int d A \int d A^{\prime} \chi_{i_{z}^{\prime} s_{s}^{\prime}}^{R^{\prime} \dagger}\left(A^{\prime}\right) \chi_{i_{x} s_{s}}^{R}(A)<\Psi\left(A^{\prime}\right)|\hat{O}| \Psi(A)>
$$

Comparing (I.39) with (I.36) makes it clear what the difference is between the quark model and chiral soliton group theoretic approaches. The discrepancy is due to the fact that, in general,

$$
<\Psi\left(A^{\prime}\right)|\hat{O}| \Psi(A)>\not \subset \delta\left(A-A^{\prime}\right)
$$

in contradistinction to (I.20). What Manohar observed is the following. A typical operator $\hat{O}$ will be a sum of operators, each of which acts on only a finite number 
of quarks at a time. As $N \rightarrow \infty$, the matrix element in (I.40) will thus contain a single-quark matrix element in which $\hat{O}$ doesn't enter, raised to a very large power. For $A^{\prime} \neq A$, this single-quark matrix element will be given by some number $\cos \theta$ less than unity, whose $N$ th power tends to zero. Hence, as $N \rightarrow \infty$, we find

$$
<\Psi\left(A^{\prime}\right)|\hat{O}| \Psi(A)>\longrightarrow \delta\left(A-A^{\prime}\right)
$$

and the chiral soliton and quark model results (so far as group theory goes) coincide!

In summary, we have good reason to believe that-insofar as a large- $N$ analysis is sensible-the physics obtained from a soliton picture of the baryon will be surprisingly close to what one would obtain from the quark model. 


\section{SYSTEMATICS OF $\pi N$ SCATTERING IN CHIRAL SOLITON MODELS}

In this chapter we shall examine some implications of the chiral soliton picture of nucleons for the real world. At leading order in $1 / N$, this approach will be shown to imply a set of energy-independent linear relations between pion-nucleon scattering amplitudes in various channels of isospin and angular momentum, as recently noted by Hayashi et al. ${ }^{[18]}$ in their analysis of $\pi N$ scattering in the Skyrme model. These relations can be used, for example, to express the isospin- $\frac{3}{2} \pi N$ elastic scattering amplitudes as linear combinations of the isospin- $\frac{1}{2}$ amplitudes in the same partial wave. Similar relations will be shown to hold for the process $\pi N \rightarrow \pi \Delta$. We shall find that in Nature these relations seem to be satisfied fairly well for most partial waves. Furthermore we shall argue that the handful of channels for which the relations are grossly disobeyed are precisely the ones most sensitive to higher-order corrections, which we have not attempted to calculate.

In addition, we shall see that the soliton interpretation of baryons provides a coherent framework for understanding some general features of the $\pi N \rightarrow \pi N$ partial wave amplitudes. In particular it offers a simple explanation for a surprisingly consistent pattern that emerges for when the four independent amplitudes in a given partial wave $L$ are compared: namely, the amplitudes corresponding to $(I, J)=\left(\frac{1}{2}, L-\frac{1}{2}\right)$ or $\left(\frac{3}{2}, L+\frac{1}{2}\right)$ are characterized by much bigger excursions through the unitarity circle than those with $(I, J)=\left(\frac{1}{2}, L+\frac{1}{2}\right)$ or $\left(\frac{3}{2}, L-\frac{1}{2}\right)$. (Here $I$ and $J$ denote the total isospin and angular momentum of the pion-nucleon system.) Furthermore, the chiral soliton picture gives an intuitive understanding for why the $S$-, $P$ - and $D$-waves are characterized both by enormous, low-lying resonances in some channels and marked repulsive behavior near threshold in others (this point will be made clear in Chapter III).

We begin our exposition in Section II.1 with a general discussion of $\pi N$ scattering in the large- $N$ limit. Here we set out our approximations and justify them as appropriate to a systematic analysis to leading order in $1 / N$. In Section 
II.2, we employ these approximations to derive the general structure of the spinand isospin-dependence of $\pi N$ amplitudes implied by the $1 / N$ expansion. Our results agree with those obtained in Ref. 18 by somewhat different means; the lengthier derivation given here has the advantage of highlighting the various points at which we invoke the large- $N$ approximation, which we hope will help pave the way for a higher-order calculation. We recover the linear relations of Ref. 18 for elastic scattering and present new relations among partial wave amplitudes for the process $\pi N \rightarrow \pi \Delta$. Finally, in Sections II.3 and II.4, we apply these relations to the experimentally determined partial wave amplitudes, considering $\pi N \rightarrow \pi N$ and $\pi N \rightarrow \pi \Delta$, respectively.

\section{Assumptions, Approximations, and Apologies}

Most of the approximations we will make relate in one way or another to the large- $N$ expansion. Our major approximation will be that of deriving the $\pi N \rightarrow \pi N$ and $\pi N \rightarrow \pi \Delta$ amplitudes from the lowest-order pion propagator in the (appropriately rotated) soliton background, ignoring all loop contributions to the two-point function. Loop diagrams necessarily contain 3-meson, 4-meson or higher-order vertices which are damped by increasingly higher powers of $f_{\pi}^{-1} \sim$ $N^{-\frac{1}{2}}$. Consequently, all loop contributions to the propagator are suppressed by at least one power of $N$ and can therefore be disregarded in our lowest-order treatment. The resulting Euler-Lagrange equations of motion for the pion field will be linear, in agreement with Witten's result from large- $N \mathrm{QCD}$, as discussed in Section I.2.

The fact that the bare pion propagator has enough structure to lead to nontrivial scattering is noteworthy and deserves some comment. On the one hand, this should be expected from the soliton picture, since meson-soliton scattering normally appears at zeroth order in a weak-coupling expansion. On the other hand, this fact implies that, in large- $N$, baryon resonances are not at all the counterparts of excited mesons. As noted in Chapter I, the widths of all excited mesons vanish like $N^{-1}$ as $N \rightarrow \infty$. Among the baryons found in Nature, how- 
ever, only the nucleon and delta (and perhaps a handful of others: see Section III.2) appear as sharply defined states in this limit. Higher-mass baryons cannot be identified with narrow states; they appear only as resonances above threshold in the various channels of pion-nucleon scattering. The widths of these baryons are determined by the motion of the $\pi N$ phase shifts in the relevant partial waves; since the equations for $\pi N$ scattering have a definite, finite large- $N$ limit, both the widths and the excitation energies of these resonances will be of order $N^{0}$.

This picture contrasts sharply with the quark model description of baryon resonances. One may think of the quark model as representing the leading term in a nonrelativistic approximation to the baryon and meson states. In this limit, unlike that of large $N$, the baryon resonances appear as eigenstates of a Hamiltonian and hence are stable to lowest order. The first nonzero contribution to their widths arises from the corrections to this approximation involving the creation of extra quark-antiquark pairs.

The major limitation of our lowest-order large- $N$ analysis is that it is appropriate only to elastic or quasielastic $\pi N$ scattering. Multiple production of pions is formally suppressed by powers of $1 / N$; nevertheless, in Nature it becomes the dominant feature of $\pi N$ scattering at high energies. Our analysis, on the other hand, allows a pion to scatter inelastically from a nucleon only by producing a rotationally excited state of the soliton. This may be a delta, with $I=J=\frac{3}{2}$, or a specific higher excitation, peculiar to chiral soliton models, with $I=J=\frac{5}{2}$, as discussed further in Section II.2.

Our second approximation will consist of ignoring the rotation of the soliton during the scattering process. As we reviewed in Chapter I, nucleons and deltas correspond in the chiral soliton models to rotating solitons ${ }^{[18]}$ of angular momentum $\mathbf{J}^{2}=i(i+1)$, with $i=\frac{1}{2}$ and $i=\frac{3}{2}$, respectively. The nucleon-delta mass-difference is then simply due to to the rotational kinetic energy term $\mathrm{J}^{2} / 2 I$, where $I$ denotes the moment of inertia of the soliton. Since $I \sim N$ this mass splitting is a $1 / N$ effect. The rotational frequency of the soliton is then given by 
$\omega=J / I$, which likewise vanishes like $1 / N$ for large $N$, thereby justifying our approximation in this limit.

This argument might not appear particularly compelling when applied to the real world, where $N=3$. However one can reverse the above relations and solve for $\omega$ in terms of $m_{N}$ and $m_{\Delta}$; the result is $\omega=\frac{2}{3} J\left(m_{\Delta}-m_{N}\right)$. The ratio of the time it takes a pion of velocity $v$ to cross the charge radius $R$ of a nucleon to the period of rotation of the nucleon viewed as a soliton is then $(v / c)^{-1}(\omega R / 2 \pi) \approx \frac{1}{16}(v / c)^{-1}$, while the corresponding ratio in the case of the delta is roughly $\frac{1}{5}(v / c)^{-1}$. Thus our approximation appears to be a reasonable one for $\pi N \rightarrow \pi N$ except near threshold, whereas for $\pi N \rightarrow \pi \Delta$ it is somewhat more severe.

Finally, we will ignore both the deformation and the recoil of the soliton. This, too, is formally justified for large $N$, since in this limit the baryon is much more massive than the pion. But in Nature this would seem to be a drastic assumption if one wants to go up to typical resonance energies, say $1700 \mathrm{MeV}$. Curiously, the linear relations among $\pi N \rightarrow \pi N$ scattering amplitudes work better and better for higher energies for a reason that has nothing to do with the validity of the large $N$ limit; we will discuss this point in Section II.3. But it is remarkable that in the Skyrme-model calculation of Chapter III, in which the same approximations are invoked, locations of resonances of very high mass (up to $3 \mathrm{GeV}$ ) are, if anything, obtained more accurately than for the lighter ones.

For $\pi N \rightarrow \pi \Delta$ this last approximation is even harder to justify. In that case, the linear relations derived in Section II.2 are only valid on an unphysical line in momentum space for which, not only are the initial and final baryons both at rest, but in addition the momenta of the incoming and outgoing pions are equal. When the final baryon is a nucleon we can get arbitrarily close to this line by considering pions sufficiently near threshold, but this is obviously not the case when the final baryon is a delta. 


\section{Derivation of Linear Relations}

We begin our analysis of pion-nucleon scattering from Skyrme's assumption that the solitons associated with the Lagrangian F.1 have the hedgehog form:

$$
U_{\circ}(\vec{x})=e^{i F(r) \widehat{r} \cdot \vec{\sigma}}
$$

As discussed in Chapter I, if $F(r)$ tends to 0 as $r \rightarrow \infty$ and to $\pi$ as $r \rightarrow 0$, this defines a configuration with topological charge equal to unity-a baryon. This configuration is maximally symmetric in the sense that, although it is not invariant with respect to isospin or spatial rotations separately, it is invariant under a combination of space and isospace rotations.

We shall use the symbols $\vec{i}$ and $\vec{s}$ to denote the isospin and angular momentum of the baryon alone, and reserve $\vec{I}$ and $\vec{J}$ for the pion-baryon system, i.e., $\vec{I}=$ $\vec{i}+\vec{I}($ pion) and $\vec{J}=\vec{s}+\vec{L}$ (pion). In this language, the soliton is transformed both by $\vec{i}$ and by $\vec{s}$, but is preserved by $\vec{i}+\vec{s}$. We shall see the significance of this peculiar symmetry in a little while, when we consider the expansion of $\mathcal{L}$ about the chiral soliton and identify the fluctuations with pions; the result is that the pion wavefunctions decompose into eigenstates of the operator $\vec{K} \equiv$ $\vec{I}($ pion $)+\vec{L}($ pion $)$.

Of course, $U_{0}$ as it stands is not a suitable candidate for a nucleon or delta; this is because physical baryons are characterized by definite values of $\vec{i}$ and $\vec{s}$ individually. One obtains a state with the correct quantum numbers by introducing collective coordinates $A(t)$, as reviewed in Section I.1. Note that the wavefunctions $\chi(A)$ given in Eq. (I.26) are really only appropriate for baryons at rest; however, as we have discussed in Section II.1, we plan in any event to neglect the baryon's recoil in the scattering process.

We shall represent pion field fluctuations about the classical soliton by letting

$$
F(r) \hat{\mathbf{r}} \rightarrow F(r) \hat{\mathbf{r}}+\frac{2}{f_{\pi}} \vec{\pi}(\vec{x}, t)
$$

in the exponent of (II.1). Expanding Eq. $F .1$ in powers of the pion field yields 
an action

$$
S=-\int d t m_{\circ}+\int d^{4} x \pi^{i *}(x) \hat{\mathrm{L}}_{i j} \pi^{j}(x)+O\left(\pi^{3} / f_{\pi}\right)
$$

where $m_{0}$ is the mass of the soliton and $i$ and $j$ are isospin indices that we shall think of as running over $(1,2,3)$ or $(+, 0,-)$ as convenient. $\widehat{\mathbf{L}}$ is accordingly a $3 \times 3$ matrix of differential operators formed from various products of $\partial_{i}, \partial_{t}^{2}, \widehat{\mathbf{r}}_{i}$ and $\delta_{i j}$. That is,

$$
\hat{\mathbf{L}}_{i j}=G_{1}(r) \delta_{i j}+G_{2}(r) \delta_{i j} \partial_{t}^{2}+G_{3}(r) \hat{r}_{i} \hat{r}_{j} \partial_{t}^{2}+G_{4}(r) \hat{r}_{j} \partial_{i}+G_{5}(r) \hat{r}_{i} \partial_{j}+\cdots
$$

with the $G_{k}(r)$ being, in general, horrible, model-dependent functions of the soliton profile $F(r)$ and its derivatives. We note that, with no loss of generality, $\hat{\mathbf{L}}$ can be chosen uniquely to be self-adjoint. As discussed in Section II.1, we shall henceforth neglect all $O\left(\pi^{3} / f_{\pi}\right)$ terms in keeping with our lowest-order approach.

We can now imagine constructing the pion propagator $\left\langle\pi^{i}\left(\vec{x}^{\prime}, t^{\prime}\right) \pi^{j}(\vec{x}, t)\right\rangle_{\circ}$ in the soliton background by the following procedure: The pion field is expanded in terms of a complete set of appropriately normalized eigenfunctions $\psi_{\lambda}^{j}(\vec{x}, t)$ satisfying

$$
\widehat{\mathbf{L}}_{i j} \psi_{\lambda}^{j}=a(\lambda) \psi_{\lambda}^{i}
$$

The propagator is then given, somewhat schematically, by

$$
\left\langle\pi^{i}\left(x^{\prime}\right) \pi^{j}(x)\right\rangle_{\circ}=\sum_{\lambda} \frac{\psi_{\lambda}^{i}\left(x^{\prime}\right) \psi_{\lambda}^{j}(x)}{a(\lambda)}
$$

The 'nought' on the propagator will serve to remind us that (II.6) represents pion-scattering from a fixed soliton in its standard orientation (II.1).

It is obvious how to generalize this formula to the case of a rotated soliton $U_{A}$ as defined in Eq. (I.18). Pion fluctuations are naturally incorporated by 
letting $F(r) D^{1}(A) \hat{\mathbf{r}} \rightarrow F(r) D^{1}(A) \widehat{\mathbf{r}}+\frac{2}{f_{\pi}} \vec{\pi}(\vec{x}, t)$ as before. This results in $\widehat{\mathbf{L}} \rightarrow$ $D^{1}(A) \hat{\mathbf{L}} D^{1}(A)^{-1}, \psi_{\lambda}^{j} \rightarrow D^{1}(A)_{j k} \psi_{\lambda}^{k}$, and thus

$$
\left\langle\pi^{i} \pi^{j}\right\rangle_{\circ} \rightarrow\left\langle\pi^{i} \pi^{j}\right\rangle_{A}=D^{1}(A)_{i m}\left\langle\pi^{m} \pi^{n}\right\rangle_{0} D^{1}(A)_{n j}^{-1}
$$

We should point out that this step assumes the rotation $A$ to be time-independent; thus, it is valid only in the limit in which the soliton does not rotate significantly during the scattering process. This approximation was justified in Section II.1 as arising from the $1 / N$ expansion.

It is now a simple matter to express in this framework the Green's functions for the "real-world" processes $\pi N \rightarrow \pi N$ and $\pi N \rightarrow \pi \Delta$. If we represent the initial and final baryons by the wavefunctions $\chi_{i_{x} s_{x}}^{R}(A)$ and $\chi_{i_{x}^{\prime} s_{x}^{\prime}}^{R^{\prime}}(A)$, respectively, then the Green's functions are given by

$$
\begin{gathered}
\left\langle\pi^{i} \pi^{j}\right\rangle=\int d A \chi_{i_{z}^{\prime} s_{x}^{\prime}}^{R^{\prime} *}(A) D^{1}(A)_{i m}\left\langle\pi^{m} \pi^{n}\right\rangle_{\circ} D^{1}(A)_{n j}^{-1} \chi_{i_{x} s_{x}}^{R}(A) \\
=\frac{1}{2 \pi^{2}} \sqrt{(2 R+1)\left(2 R^{\prime}+1\right)} \sum_{m n}\left\langle\pi^{m} \pi^{n}\right\rangle_{0} \\
\times \int d A\left(D^{R^{\prime}}(A) \epsilon^{R^{\prime}}\right)_{i_{s}^{\prime} s_{s}^{\prime}} D^{1}(A)_{i m} D^{1}(A)_{n j}^{-1}\left(\epsilon^{R} D^{R}(A)^{-1}\right)_{s_{x} i_{x}}
\end{gathered}
$$

where we have substituted the explicit expressions for the baryon wavefunctions given in Eq. (1.26).

It turns out that the $A$-integration in Eq. (II.8) can be carried out explicitly. The most compact expression for the Green's function is then obtained by projecting the initial and final pion-baryon states onto states of definite total isospin and angular momentum $\left|I I_{z} J J_{z}\right\rangle .(\vec{I}$ and $\vec{J}$ are of course conserved in the scattering process.) Following convention, we further restrict the incoming and outgoing pions to partial waves $L$ and $L^{\prime}$, respectively, while the initial and final baryons are characterized by spin (and isospin) $R$ and $R^{\prime}$ as before. These steps 


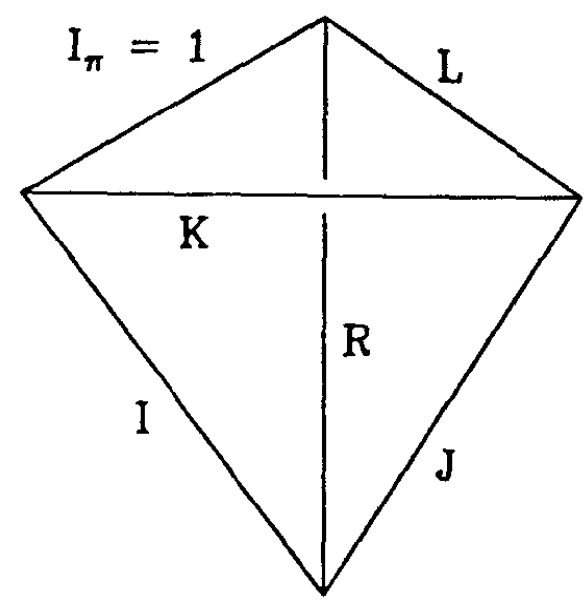

Fig. 6. Relation of the six coupled angular momenta in either the initial or the final state of pion-skyrmion scattering

are carried out in detail in Appendix A. The appropriately projected pion Green's functions, which we label $\mathbf{G}_{L L^{\prime} R R^{\prime} \bar{I} J^{J}}\left(r t ; r^{\prime} t^{\prime}\right)$, are then given by an expression of the form:

$$
\mathrm{G}_{L L^{\prime} R R^{\prime} \vec{I} \vec{J}}=\sum_{K} P_{L L^{\prime} R R^{\prime} I J K} \cdot \mathbf{g}_{K L^{\prime} L}\left(r t ; r^{\prime} t^{\prime}\right)
$$

The meanings of the terms on the right-hand side of this expression are as follows: $\mathrm{g}_{K L^{\prime} L}\left(r t ; r^{\prime} t^{\prime}\right)$ is the "reduced" radial Green's function describing elastic pion-scattering from the "elementary" soliton (II.1), where the incoming and outgoing pions are restricted to partial waves $L$ and $L^{\prime}$, respectively; this restriction leaves a purely radial scattering problem. The index $K$ denotes the conserved quantum number of this "elementary" process, which is the vectorial sum $\vec{K}=\vec{I}($ pion $)+\vec{L}$ (pion). Note that, by the triangle inequality, $K$ is restricted to the values $\max \left(|L-1|,\left|L^{\prime}-1\right|\right) \leq K \leq \min \left(L+1, L^{\prime}+1\right)$. Finally, the $P$ symbols are group-theoretic coefficients calculated in Appendix A:

$$
P_{L L^{\prime} R R^{\prime} I J K}=(-1)^{R^{\prime}-R} \sqrt{(2 R+1)\left(2 R^{\prime}+1\right)}(2 K+1)\left\{\begin{array}{c}
K I J \\
R^{\prime} L^{\prime} 1
\end{array}\right\}\left\{\begin{array}{c}
K I J \\
R L 1
\end{array}\right\} .
$$


The appearance of $6 j$-symbols in this expression is quite natural, since, as indicated in Fig. 6, the problem in both the entering and the exiting $\pi N$ channels is characterized by six intertwined angular momenta. Explicit formulas for the $P$-symbols relevant to $\pi N \rightarrow \pi N$ and $\pi N \rightarrow \pi \Delta$ are presented in Appendix B. Note that all the model dependence arising from the details of the Lagrangian $F .1$ is subsumed in the quantities $\mathbf{g}_{K L^{\prime} L}$; the $P$-symbols, in contrast, depend only on the hedgehog nature of the chiral soliton.

Since $6 j$-symbols embody various triangle inequalities, the same is true for the $P$-symbols. Specifically, the seven triads $(R 1 I),\left(R^{\prime} 1 I\right),(R L J),\left(R^{\prime} L^{\prime} J\right)$, $(L 1 K),\left(L^{\prime} 1 K\right)$ and $(I J K)$ must each satisfy the triangle inequality in order for the $P$-symbol not to vanish (cf. Fig. 6). Of these triads, the first four merely express the obvious bounds on the total isospin and angular momentum formed from a baryon of spin and isospin $R$ (or $R^{\prime}$ ) and a pion with orbital angular momentum $L$ (or $L^{\prime}$ ).

As for $(L 1 K)$ and $\left(L^{\prime} 1 K\right)$, these reflect the existence of the conserved vector $\vec{K}$ in processes in which a pion scatters off an elementary soliton. Indeed Eq. (II.9) can best be regarded as an expansion of the physical processes $\pi N \rightarrow \pi N$ or $\pi N \rightarrow \pi \Delta$ in terms of these elementary channels, each labeled by its own value of $K$. The emergence of this new quantum number as a quantity of physical import is of course peculiar to models that admit solitons of the form given in Eq. (II.1). The final triad ( $I J K)$ is something of a surprise. (In fact it is the only one of the seven triangle inequalities not already manifest in the Clebsch-Gordon coefficients of Eq. (A.9), which is the penultimate formula in the derivation of (II.9).) In practice, it frequently serves to eliminate one of the (typically three) elementary channels associated with fixed $K$ which would normally be expected to contribute to a given $(I, J, L)$ channel of physical pion-baryon scattering.

Despite these restrictions it turns out that Eq. (II.10) does not prohibit any $\pi N$ or $\pi \Delta$ processes otherwise allowed by parity, isospin, and angular momentum. For example, $(L 1 K)$ and $\left(L^{\prime} 1 K\right)$ taken together forbid jumps in pion angular 
momentum greater than two; but $\Delta L \geq 3$ is in any event excluded by parity and/or angular momentum conservation.

The relation (II.9) for Green's functions can be immediately converted to a relation for $S$-matrix elements by moving onto the pion mass-shell and extracting the pole term on each side of this equation. Modulo the extrapolation from an unphysical region in momentum-space as discussed in Sec. II.1, these manipulations do not change the form of the relation, and we have:

$$
\mathrm{S}_{L L^{\prime} R R^{\prime} \vec{I} \vec{J}} \equiv^{+}\left\langle R^{\prime} \vec{I} \vec{J} L^{\prime} \mid R \vec{I} \vec{J} L\right\rangle^{-}=\sum_{K} P_{L L^{\prime} R R^{\prime} I J K} \cdot \mathbf{s}_{K L^{\prime} L}
$$

We will refer to the $\mathbf{s}_{K L^{\prime} L}$ as the reduced $S$-matrix. Notice that a resonance in some elementary channel $\left(K L L^{\prime}\right)$ manifests itself in $\pi N$ scattering as a family of resonances coupling to that value of $K$. It is thus appropriate in the chiral soliton picture to classify resonances according to $K$. This scheme replaces the conventional $S U(6)$ (or, in the 2-flavor case, $S U(4)$ ) classification of baryon resonances. We should recall, though, that these resonances should not be considered narrow, so that one may not ignore background contributions from other values of $K$ coupling to the same physical partial wave amplitude.

As a check on (II.11) let us verify the unitarity condition

$$
+\langle f \mid i\rangle^{-}=\sum_{\psi}+\langle f \mid \psi\rangle^{\circ \circ}\langle\psi \mid i\rangle^{-}
$$

where $|\psi\rangle^{\circ}$ runs over a complete set of states at an intermediate time. Accordingly we rewrite the reduced $S$-matrix as

$$
\mathbf{s}_{K L^{\prime} L} \equiv \mathbf{s}_{K L^{\prime} L}^{+-} \equiv^{+}\left\langle K L^{\prime} \mid K L\right\rangle^{-}
$$

and insert

$$
\sum_{R^{\prime \prime} L^{\prime \prime}}\left|R^{\prime \prime} \vec{I} \vec{J} L^{\prime \prime}\right\rangle^{\circ}{ }^{\circ}\left\langle R^{\prime \prime} \vec{I} \vec{J} L^{\prime \prime}\right|
$$


into (II.11). Taking advantage of the fact that

$$
\sum_{R^{\prime \prime}} P_{L L^{\prime \prime} R R^{\prime \prime} I J K^{\prime}} P_{L^{\prime \prime} L^{\prime} R^{\prime \prime} R^{\prime} I J K}=\delta_{K K^{\prime}} P_{L L^{\prime} R R^{\prime} I J K}
$$

(which holds so long as the triad $\left(L^{\prime \prime} 1 K\right)$ satisfies the triangle inequality), we obtain the constraint

$$
\sum_{K} P_{L L^{\prime} R R^{\prime} I J K} \cdot\left(\mathrm{s}_{K L^{\prime} L}^{+-}-\sum_{L^{\prime \prime}} \mathrm{s}_{K L^{\prime} L^{\prime \prime}}^{+\circ} \mathrm{s}_{K L^{\prime \prime} L}^{\circ-}\right)=0
$$

where we have set $+\left\langle K L^{\prime} \mid K L^{\prime \prime}\right\rangle^{\circ}=\mathrm{s}_{K L^{\prime} L^{\prime \prime}}^{+\circ}$, etc. From this we can draw the reassuring conclusion that if the reduced $S$-matrix obeys (II.12) (as surely it must), then the physical amplitudes are guaranteed to do so as well.

Note, however, that it is generally necessary to include the "exotic" baryons with $R^{\prime \prime}>3 / 2$ called for by the model among the states of the complete set (II.14). This should not be surprising; the chiral soliton model contains a state of spin and isospin $\frac{5}{2}$ as the second rotational excitation of the nucleon, and there is no selection rule forbidding the production of this baryon in isospin- $\frac{3}{2} \pi N$ scattering, via the process $\pi N \rightarrow \pi B_{\frac{5}{2}}$. By analogy with the rigid rotor, the mass of this baryon would satisfy $\left(m_{\frac{5}{2}}-m_{N}\right) /\left(m_{\Delta}-m_{N}\right)=\frac{8}{3}$, or $m_{\frac{5}{2}} \simeq 1720 \mathrm{MeV}$. Of course, in Nature there is no such state narrow enough to be distinguished. It is nevertheless conceivable that a very broad $i=s=\frac{5}{2}$ resonance exists. Presumably it would decay mostly into $\Delta \pi$ and would therefore show up obliquely in Nature as an enhancement of the $\Delta \pi \pi$ final state in pion-nucleon scattering.

The obvious benefit of Eq. (II.11) is that it decomposes a large number of physical scattering amplitudes in terms of a substantially smaller set of reduced amplitudes. Consequently it is possible to eliminate the latter and be left with nontrivial energy-independent linear relations between physical amplitudes. Before doing so, however, we pause briefly to take note of two general constraints on the the reduced amplitude $s_{K} L^{\prime} L$. First of all, parity conservation together 
with the triangle inequalities discussed above for $(L 1 K)$ and $\left(L^{\prime} 1 K\right)$ imply that either $L=L^{\prime}$ or $L=L^{\prime} \pm 2$. Secondly, it follows from time-reversal invariance and unitarity that the $S$-matrix is symmetric: ${ }^{[23]}$

$$
\mathbf{8}_{K L^{\prime} L}=\mathbf{8}_{K L L^{\prime}}
$$

Bearing these constraints in mind, we can now straightforwardly find linear combinations of the left-hand side of (II.11) so that the model-dependent right-hand side cancels out.

We focus first on the case $\pi N \rightarrow \pi N$; in our notation this implies $L=L^{\prime}$ and $R=R^{\prime}=\frac{1}{2}$. We choose to solve for the $I=\frac{3}{2}$ amplitudes in terms of those with $I=\frac{1}{2}$. From the explicit formulas for the $P$-symbols as given in Appendix B one obtains:

$$
\begin{gathered}
\mathbf{S}_{L L \frac{1}{2} \frac{1}{2} \frac{3}{2}, L-\frac{1}{2}}=\frac{L-1}{4 L+2} \cdot \mathbf{S}_{L L \frac{1}{2} \frac{1}{2} \frac{1}{2}, L-\frac{1}{2}} \\
+\frac{3 L+3}{4 L+2} \cdot \mathbf{S}_{L L \frac{1}{2} \frac{1}{2} \frac{1}{2}, L+\frac{1}{2}}
\end{gathered}
$$

and

$$
\begin{gathered}
\mathbf{S}_{L L \frac{1}{2} \frac{1}{2} \frac{3}{2}, L+\frac{1}{2}}=\frac{3 L}{4 L+2} \cdot \mathbf{S}_{L L \frac{1}{2} \frac{1}{2} \frac{1}{2}, L-\frac{1}{2}} \\
+\frac{L+2}{4 L+2} \cdot \mathbf{S}_{L L \frac{1}{2} \frac{1}{2} \frac{1}{2}, L+\frac{1}{2}}
\end{gathered}
$$

These relations were also derived in Ref. 18 .

For $\pi N \rightarrow \pi \Delta$ we can have either $L=L^{\prime}$ or $L=L^{\prime} \pm 2$ consistent with angular momentum conservation. For $L=L^{\prime}$ we find:

$$
\begin{aligned}
\mathbf{S}_{L L \frac{1}{2} \frac{3}{2} \frac{3}{2}, L-\frac{1}{2}} & =\frac{4(L-1)}{\sqrt{10}(2 L+1)} \cdot \mathbf{S}_{L L \frac{1}{2} \frac{1}{2}, L-\frac{1}{2}} \\
& +\frac{3}{2 L+1} \sqrt{\frac{(L+1)(2 L+3)(2 L-1)}{10 L}} \cdot \mathbf{S}_{L L \frac{1}{2} \frac{1}{2} \frac{1}{2}, L+\frac{1}{2}}
\end{aligned}
$$


and likewise

$$
\begin{aligned}
S_{L L \frac{1}{2} \frac{8}{2} \frac{3}{2}, L+\frac{1}{2}}= & \frac{3}{2 L+1} \sqrt{\frac{L(2 L+3)(2 L-1)}{10(L+1)}} \cdot S_{L L \frac{1}{2} \frac{8}{2} \frac{1}{2}, L-\frac{1}{2}} \\
& +\frac{4(L+2)}{\sqrt{10}(2 L+1)} \cdot \mathbf{S}_{L L \frac{1}{2} \frac{8}{2} \frac{1}{2}, L+\frac{1}{2}}
\end{aligned}
$$

while for $L=L^{\prime} \pm 2$ we obtain the simple proportionality relations

$$
\begin{aligned}
& \sqrt{L+1} \cdot \mathbf{S}_{L, L+2, \frac{1}{2} \frac{8}{2} \frac{1}{2}, L+\frac{1}{2}}=-\sqrt{10(L+1)} \cdot \mathbf{S}_{L, L+2, \frac{1}{2} \frac{8}{2} \frac{8}{2}, L+\frac{1}{2}} \\
& =-\sqrt{L+2} \cdot \mathbf{S}_{L+2, L \frac{1}{2} \frac{8}{2} \frac{1}{2}, L+\frac{3}{2}}=\sqrt{10(L+2)} \cdot \mathbf{S}_{L+2, L \frac{1}{2} \frac{3}{2} \frac{8}{2}, L+\frac{8}{2}} \cdot
\end{aligned}
$$

Finally, for each $L$ there is one additional linear relation which serves to relate $\pi N \rightarrow \pi N$ to $\pi N \rightarrow \pi \Delta:$

$$
\begin{aligned}
\mathbf{S}_{L L \frac{1}{2} \frac{1}{2} \frac{1}{2}, L-\frac{1}{2}} & -\mathbf{S}_{L L \frac{1}{2} \frac{1}{2} \frac{1}{2}, L+\frac{1}{2}} \\
& =\sqrt{\frac{2 L-1}{L+1}} \cdot \mathbf{S}_{L L \frac{1}{2} \frac{3}{2} \frac{1}{2}, L-\frac{1}{2}}+\sqrt{\frac{2 L+3}{L}} \cdot \mathbf{S}_{L L \frac{1}{2} \frac{3}{2} \frac{1}{2}, L+\frac{1}{2}}
\end{aligned}
$$

(Note that this relation depends on the phase convention of the delta wavefunctions vis-a-vis the nucleons.)

We turn now to an examination of how well these relations are obeyed in Nature.

\section{Comparison with Experiment: $\pi N \rightarrow \pi N$}

We focus first on the process $\pi N \rightarrow \pi N$. Elastic $\pi N$ scattering in the lowenergy regime has been the subject of thorough experimental investigation. Our analysis in this section relies on the data compilation of Höhler, et al., ${ }^{[24]}$ in which a complete partial-wave analysis of elastic $\pi N$ scattering is presented for center-of-mass energies $W$ up to $4.5 \mathrm{GeV}$. For elastic scattering the relevant linear relations are given by Eq. (II.18), which expresses the two isospin- $\frac{3}{2}$ amplitudes 

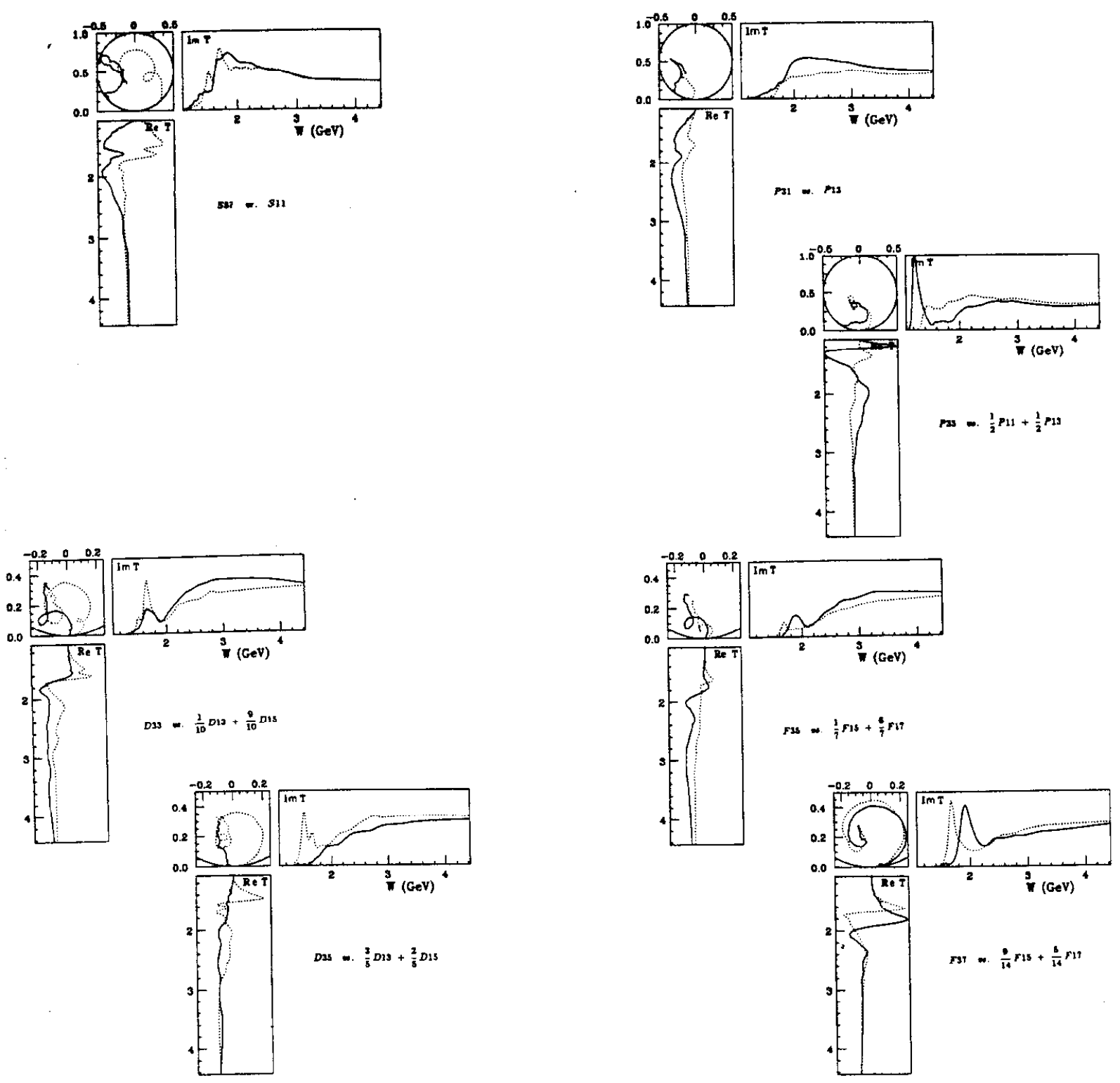

Fig. 7. Experimentally determined $I=\frac{3}{2}$ partial-wave amplitudes for $\pi N$ elastic scattering, plotted together with the linear combinations of $I=\frac{1}{2}$ amplitudes which should reproduce them if Eq. II.18 is valid. The $I=\frac{8}{2}$ amplitudes are indicated by solid lines, the $I=\frac{1}{2}$ combinations by dotted lines. We have used the values of these amplitudes presented in Ref. 24.

as linear combinations of the two isospin- $\frac{1}{2}$ amplitudes in the same partial wave. We now examine the experimental validity of these relations.

In Fig. 7, we display the experimental isospin- $\frac{3}{2} \pi N$ scattering amplitudes for 

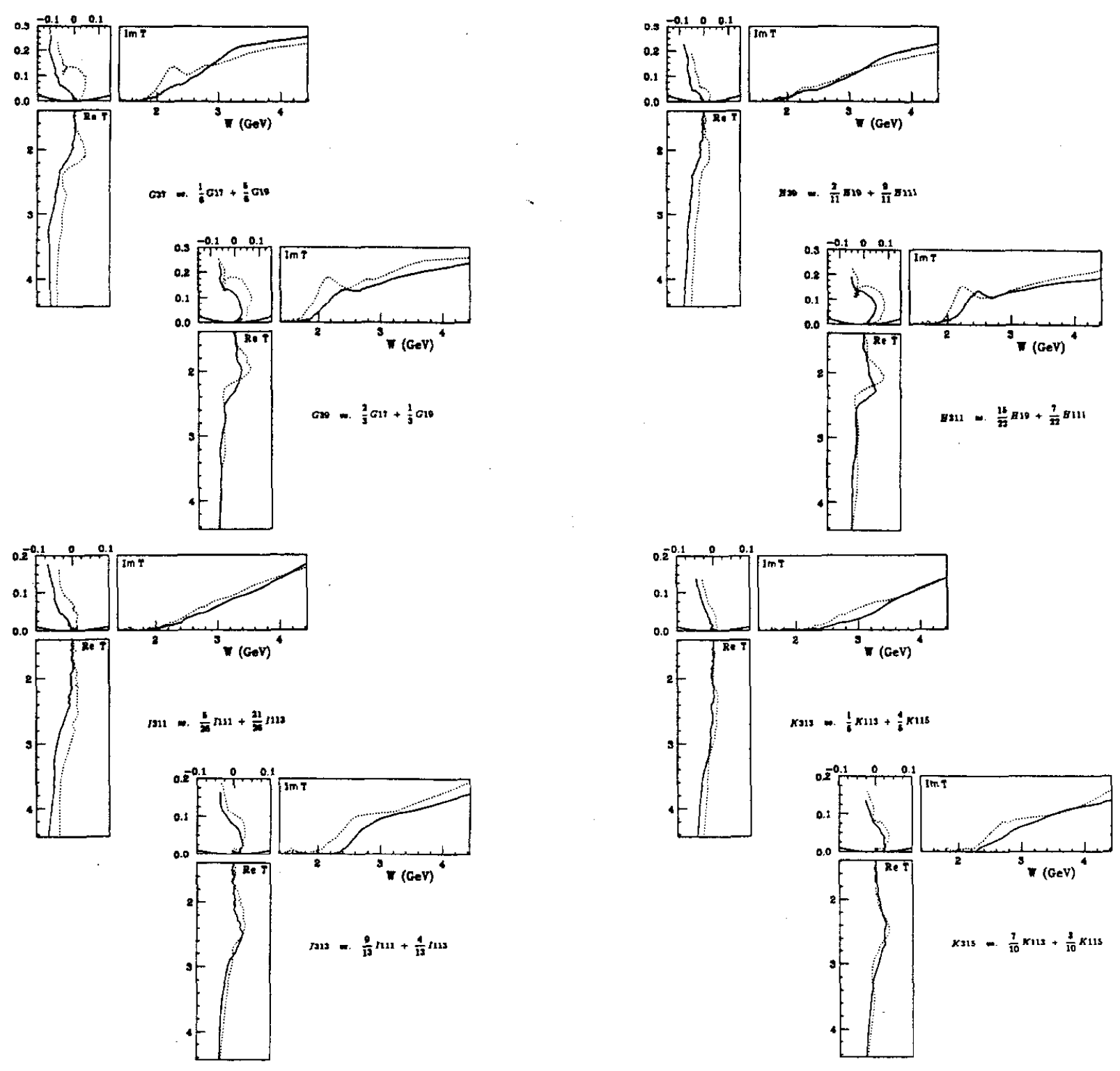

Fig. 7, cont.

$L \leq 7$ juxtaposed with those particular linear combinations of isospin- $\frac{1}{2}$ amplitudes to which they are predicted to correspond; these are indicated by solid and dashed lines, respectively. The closeness of these comparisons can be considered a model-independent test, not only of the chiral-soliton description of baryons, but also of the extent to which a lowest-order analysis in the $1 / N$ expansion can be trusted to give a reasonable description of Nature. We should point out that, 
in keeping with tradition, our Argand plots depict the $T$-matrix; this is related to the $S$-matrix via $\mathbf{T}=\frac{1}{2 i}(\mathbf{S}-\mathbf{1})$, with 1 denoting the identity operator on the Hilbert space (which vanishes for inelastic scattering). The most striking feature of the graphs taken as a whole is the substantial qualitative agreement that one finds between theory and experiment, particularly for $L \geq 3$ ( $F$-waves and higher). On a quantitative level, it turns out that, with few exceptions, the actual $I=\frac{3}{2}$ resonances are typically more massive by $150-300 \mathrm{MeV}$ than predicted by the superposed $I=\frac{1}{2}$ amplitudes. This systematic splitting is presumably caused by the same rotational energy contribution that is responsible for the nucleon-delta mass difference; since this is a $1 / N$ effect, it does not, indeed cannot, emerge in our lowest-order analysis. In contrast, it is apparent on the whole that the shapes of the resonances are correctly predicted by Eq. (II.18), and that the form of the backgrounds are reproduced quite satisfactorily. The correlation between the detailed structure of the $F_{37}$ resonance and the corresponding linear combination $\frac{9}{14} F_{15}+\frac{5}{14} F_{17}$ is particularly remarkable. Note that the background contributions tend to be given correctly even in those low- $L$ channels such as $P_{33}$ for which the structure of the resonances is not reproduced well.

Having noted the generally high degree of agreement, it is of course important to confront the disappointing results in the $S_{31}, P_{33}$ and $D_{35}$ channels. It turns out that the poor agreement in these channels is not necessarily fatal to the chiral soliton ansatz. To see this, note that in each case the discrepancies are clearly the greatest near threshold. Now, it is a property of all chiral soliton models in which the soliton is of the hedgehog form that the threshold behavior of the $S-, P$ - and $D$-wave amplitudes (and only these amplitudes) is extremely sensitive to small perturbations. This point will be argued at length in Chapter III; the upshot is that it is completely unrealistic to expect a lowest-order calculation in $1 / N$ such as ours to yield good agreement near threshold for the $S$-, $P$ - and $D$-wave amplitudes. We find it encouraging that these are the only partial waves which are in serious disaccord with Eq. (II.18) at low energies, and furthermore, that 
at higher energies (albeit still in the resonance region) the agreement markedly improves.

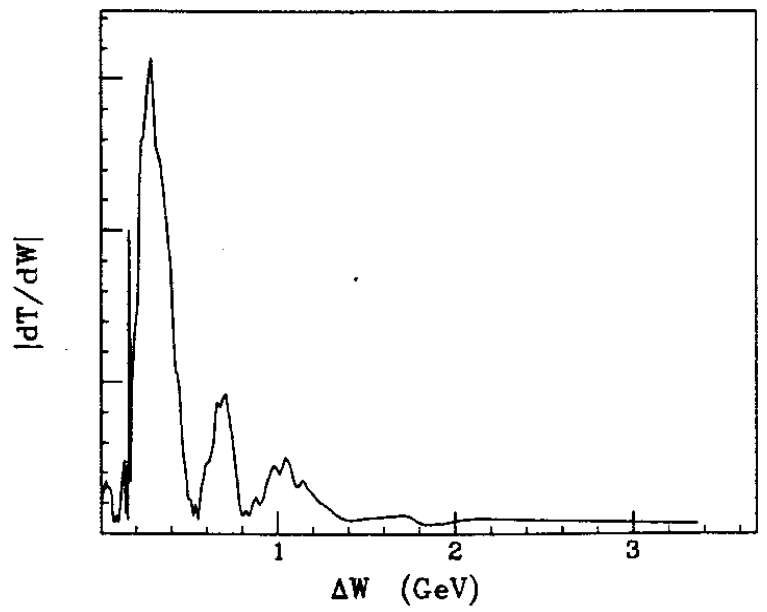

Fig. 8. Speed of motion $|d T / d W|$ of the predicted $P_{8 s} \pi N$ elastic scattering amplitude, $P_{83}^{\text {pred }}=\frac{1}{2} P_{11}+$ $\frac{1}{2} P_{23} . \Delta W$ is the energy above threshold $(1077 \mathrm{MeV})$.

As evidence for this latter claim, consider Fig. 8, where we have plotted the speed of motion $|d \mathbf{T} / d W|$ of our prediction for the $P_{33}$ partial wave amplitude as a function of the excitation energy $\Delta W$; the maxima of the speed should give the positions of resonances. In Nature, this channel is characterized by three resonances, at 1232, 1522 and $1868 \mathrm{MeV}$. Our prediction likewise gives evidence of three resonances, at 1360,1780 and $2140 \mathrm{MeV}$; the shifts from Nature are of the order of $1 / N$ corrections. The discrepancies at low energies evident in the juxtaposed graphs in this channel can be traced to the large difference in elasticity between the $\Delta(1232)$, in the true $P_{33}$ channel, and the 'Roper resonance' $P_{11}(1410)$, which contributes to the comparison curve. Now, elasticity factors are measures of the phase-space of available decay modes; consequently, near thresholds they are extremely sensitive to small shifts in mass, and hence, to higher-order $1 / N$ effects. We conclude that the agreement obtained in the $P_{33}$ channel is as good as can reasonably be expected in a lowest-order treatment. 
Before leaving the discussion of discrepancies in the low-lying partial waves, we should address the subject of the apparent violation of Weinberg's well-known calculation $^{[10]}$ of the $S$-wave scattering-lengths ${ }^{\star} a_{I=\frac{1}{2}}$ and $a_{I=\frac{8}{2}}$. The prediction is

$$
a_{I=\frac{1}{2}}=-2 a_{I=\frac{8}{2}}=\frac{g_{\mathrm{V}}^{2}}{\pi f_{\pi}^{2}} \cdot \frac{m_{\pi} m_{N}}{m_{\pi}+m_{N}}
$$

which, in particular, correctly implies that the isospin- $\frac{1}{2}$ and isospin- $\frac{3}{2} S$-wave amplitudes should exhibit attractive and repulsive behavior, respectively, near threshold. In contrast, the chiral-soliton prediction emerging from Eq. (II.18)b is that these amplitudes should be identically equal to one another! This is all the more puzzling in light of the result ${ }^{[11]}$ that chiral soliton models must necessarily obey all soft-pion theorems, of which Weinberg's is a notable example.

Actually, there is no contradiction. To see this, recall that the equality of the amplitudes implied by (II.18)b is only valid to order $N^{0}$. To this order, as we shall discuss in Chapter III, there are $S$-channel poles that sit precisely on the real axis at the nucleon mass (where they have actually coalesced with zeroes of the $S$-matrix). Now, by elementary trigonometry, a phase-shift evaluated at a given value of energy is roughly proportional to the imaginary part of the nearby pole; this implies that, to lowest order, both $a_{I=\frac{1}{2}}$ and $a_{I=\frac{3}{2}}$ must vanish identically. And indeed, the right-hand side of Eq. (II.22) is manifestly of order $1 / N$, with the result that Weinberg's prediction is trivially satisfied to order $N^{0}$. A nontrivial consistency check, then, must await a higher-order calculation.

We should comment further on the striking agreement apparent in all the channels depicted in Fig. 7 in the high-energy limit. Actually this agreement is something of an accident: in Nature, the four independent amplitudes in each partial wave become virtually degenerate at high energies (typically, $W \gtrsim 2800$ $\mathrm{MeV}$ ), with the result that Eq. (II.18) is satisfied automatically. What we

* Recall that the $S$-wave $T$-matrix is related to the scattering length $a$ near threshold via $\mathbf{T}=\frac{1}{2 i}(\exp (2 i a k)-1)$, where $k$ is the pion momentum. 
have, then, is in essence no more than a pleasing consistency check on our linear relations ${ }^{\dagger}$.

In fact, one can argue that the $1 / N$ expansion is no longer appropriate at high energies. In this regime, the $\pi N$ total cross section is dominated by multiple production of pions. The elastic amplitude (which becomes characterized more and more by forward scattering) then arises as the shadow of this multiple production via the optical theorem. In the language of Regge theory, the elastic amplitude is dominated by Pomeron exchange. The couplings of the Pomeron are independent of spin and isospin orientation; this accounts for the degeneracy mentioned above. In any case, it is clear that these couplings, arising as they do from multiple pion production, depend simultaneously on many orders in $1 / N$. Consequently, where the Pomeron dominates, a leading-order $1 / N$ analysis is necessarily inadequate. It is therefore not surprising that explicit calculations of the partial-wave amplitudes in the specific case of the Skyrme model grossly underestimate the inelasticity of $\pi N$ scattering in all partial waves at high energy, as we shall see in the following chapter.

We turn, finally, to what we consider the most compelling argument in favor of the chiral soliton ansatz that can be gleaned from an analysis of pion-nucleon scattering. We have just seen that, as the center-of-mass energy $W \rightarrow \infty$, the four independent amplitudes corresponding to each partial wave approach a common limit. However, for intermediate ranges of energy this is decidedly not the case. In fact, when one restricts $W$ to be $\leq 2.5 \mathrm{GeV}$ the experimental Argand plots exhibit a strikingly consistent pattern: for each value of $L$, the excursion of the amplitude into the unitarity circle is nearly always much larger for the $(I, J)=\left(\frac{1}{2}, L-\frac{1}{2}\right)$ or $\left(\frac{3}{2}, L+\frac{1}{2}\right)$ channels than for $\left(\frac{1}{2}, L+\frac{1}{2}\right)$ or $\left(\frac{3}{2}, L-\frac{1}{2}\right)$. We will refer to this as the "big-small-small-big" pattern. This pattern is even more pronounced if, for each $L$, one considers energies ranging up the 'natural' scale characterizing the

† It should be mentioned, however, that for the majority of channels the agreement in this region is closer by $30-50 \%$ than what one would expect from comparing to a "random" (convex) linear combination. 
resonance region of that partial wave (a precise determination of these 'natural' scales is unimportant). Certainly in a case such as this a picture is worth a thousand words; we present the relevant pictures in Fig. 9. (Not surprisingly, the only exception to the rule is in the recalcitrant $D_{35}$ channel; also, the $G_{19}$ amplitude is as large as the $G_{39}$.)

Clearly, this pattern of size alternation is consistent with Eqs. (II.18)a and (II.18) $\mathrm{b}$, since, in these equations, the $\left(\frac{3}{2}, L-\frac{1}{2}\right)$ and $\left(\frac{3}{2}, L+\frac{1}{2}\right)$ amplitudes are linked by large coefficients to the $\left(\frac{1}{2}, L+\frac{1}{2}\right)$ and $\left(\frac{1}{2}, L-\frac{1}{2}\right)$ amplitudes, respectively. But of course, the reversed pattern, with the 'small' and 'big' channels interchanged, would have been equally consistent. For a more compelling argument, one must necessarily go beyond the purely group-theoretic reasoning that led to (II.18) and add a single plausible dynamical assumption.

To this end, let us return to Eq. (II.11), in which the physical $\pi N \rightarrow \pi N$ amplitudes in the $L$ th partial wave are expressed as linear combinations of the 'reduced' amplitudes $8_{K L L}$ with $K=L-1, L, L+1$. Now, in the specific case of the Skyrme model, it turns out that the variation of $\mathbf{s}_{L+1, L L}$ away from unity is essentially negligible compared to that of $\mathbf{s}_{L-1, L L}$ and $\mathbf{s}_{L L L}$ for energies less than $2.5 \mathrm{MeV}$, as we shall see in Chapter III. Certainly it is not unreasonable to assume that this continues to be true for the 'optimal' two-flavor effective Lagrangian, especially in light of the relatively high degree of success with which the Skyrme model will be shown to reproduce the full spectrum of nucleon and delta resonances in Nature. If accordingly we make the dynamical assumption that $\mathrm{s}_{L+1, L L} \approx 1$ throughout the relevant energy ranges (ignoring inelasticities for the sake of simplicity) and represent the physical amplitude $\mathbf{S}_{L L \frac{1}{2} \frac{1}{2} I J}$ more compactly as $\mathbf{S}_{L I J}$, then Eq. (II.11) becomes: 


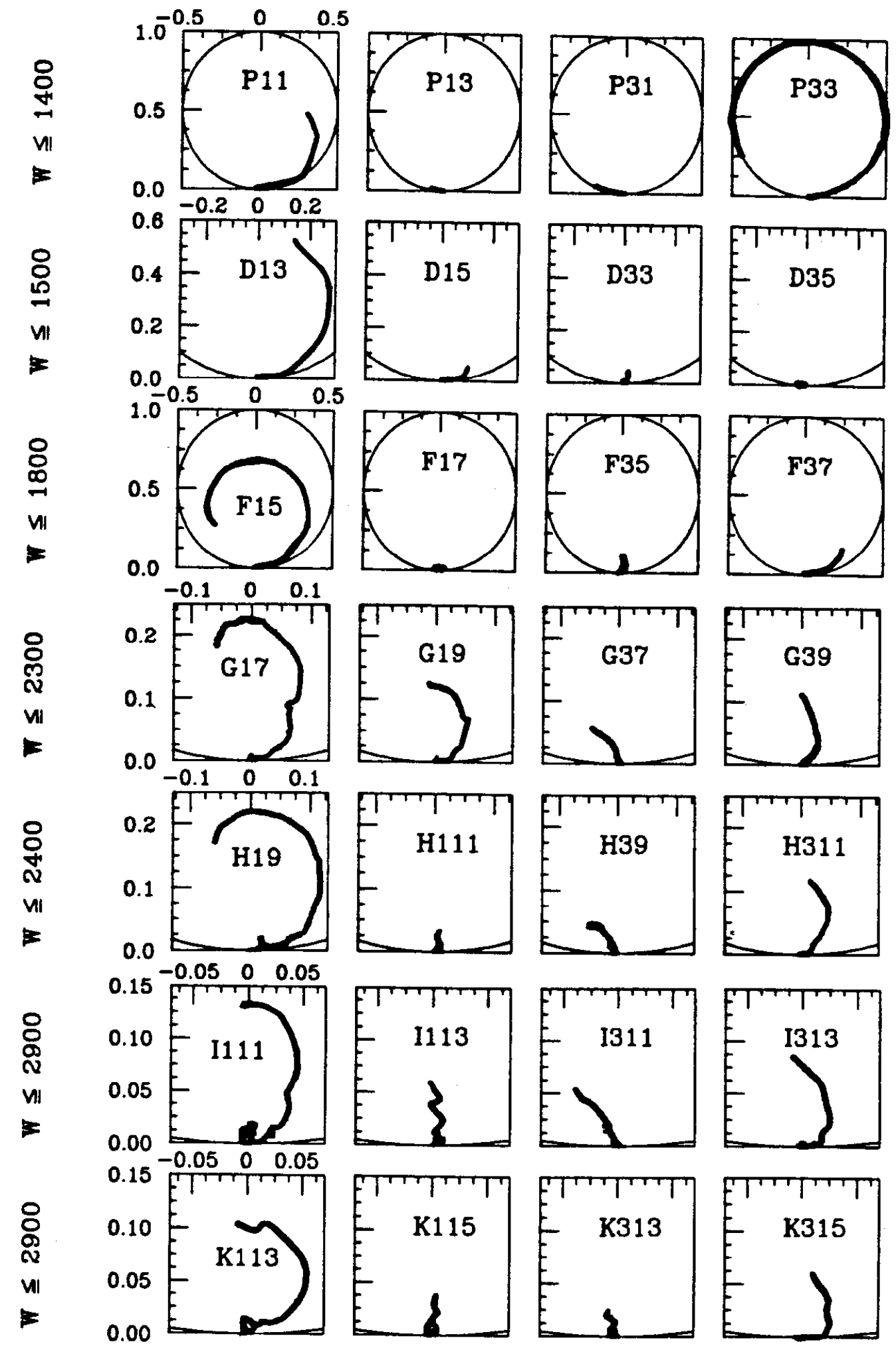

Fig. 9. Motion of the various $\pi N$ ecattering amplitndes in the unitarity circle, over a range of $W$ from threshold into the resonance region in that channel. 


$$
\begin{aligned}
& \mathrm{S}_{L \frac{1}{2}, L-\frac{1}{2}}-1=\frac{2 L-1}{3 L} \cdot\left(\mathrm{s}_{L-1, L L}-1\right)+\frac{L+1}{3 L} \cdot\left(\mathrm{s}_{L L L}-1\right), \\
& \mathrm{S}_{L \frac{1}{2}, L+\frac{1}{2}}-1=\frac{L}{3 L+3} \cdot\left(\mathrm{s}_{L L L}-1\right), \\
& \mathrm{S}_{L \frac{3}{2}, L-\frac{1}{2}}-1=\frac{(2 L-1)(L-1)}{6 L(2 L+1)} \cdot\left(\mathrm{s}_{L-1, L L}-1\right)+\frac{2 L-1}{6 L} \cdot\left(\mathrm{s}_{L L L}-1\right), \\
& \mathrm{S}_{L \frac{3}{2}, L+\frac{1}{2}}-1=\frac{2 L-1}{4 L+2} \cdot\left(\mathrm{s}_{L-1, L L}-1\right)+\frac{2 L+3}{6 L+6} \cdot\left(\mathrm{s}_{L L L}-1\right) .
\end{aligned}
$$

The pattern of alternating size now emerges as an automatic consequence of the group theory: it is simply due to the relatively small coefficients in the middle two equations of (II.23) as compared to the outer two! A further prediction of these expressions is that, of the two 'big' amplitudes, $\mathbf{S}_{L \frac{1}{2}, L-\frac{1}{2}}$ should dominate $\mathbf{S}_{L \frac{s}{2}, L+\frac{1}{2}}$-and, with the single exception of the $P$-channels, this is also apparent in Fig. 9.

\section{Comparison with Experiment: $\pi N \rightarrow \pi \Delta$}

We conclude this chapter with a brief examination of the inelastic process $\pi N \rightarrow \pi \Delta$ in the chiral soliton framework. We should remark at the outset that this process constitutes a much more tenuous proving ground for the chiral soliton ansatz than the elastic case: On the one hand, the extraction of partial-wave amplitudes from experiment requires a nontrivial and model-dependent analysis to disentangle $\pi \Delta$ from a variety of other final states such as $\rho N, \epsilon N$ and $\pi N^{*}$. On the other hand, from a theoretical point of view, several of the approximations we have invoked in the derivation of the linear relations become substantially more drastic in the inelastic case, as we have discussed in Section II.1. Throughout this section we draw from the recent partial-wave analysis of Manley et al. ${ }^{[14]}$; the $\pi \Delta$ data presented there is restricted to $W \leq 2 \mathrm{GeV}$ and $L \leq 3$.

* The analysis of ref. 14 presents the values of the partial-wave amplitudes derived from an energy-independent analysis and a unitary, energy-dependent fit to this values. Because the directly extracted amplitudes are often sparse and erratic, we have chosen to use the fit in making our comparison. This fit is generally a good representation of the elementary 
We begin by looking at processes in which the pion jumps two units of angular momentum. From Eq. (II.20) we predict simple proportionality relations between partial-wave amplitudes: ${ }^{\dagger}$

$$
S D_{11}=-\sqrt{2} \cdot D S_{13}=-\sqrt{10} \cdot S D_{31}=\sqrt{20} \cdot D S_{33}
$$

and

$$
F P_{15}=-\sqrt{10} \cdot F P_{35}
$$

These relations are checked in Fig. 10. For the $S D$ and $D S$ waves, the agreement is not impressive. The relative signs of the four amplitudes are predicted correctly, but there is no evidence for the factor of $\sqrt{10}$ which connects the first and second pairs of terms in Eq. (II.24)a. One should note, of course, that these channels all couple to the translation zero-modes. For the $F P$ waves, which do not, the agreement is quite satisfactory, up to the customary $150-200 \mathrm{MeV}$ energy shift between the isospin $-\frac{1}{2}$ and the isospin $-\frac{3}{2}$ amplitudes.

We turn next to processes for which the initial and final pion angular momenta are equal. In both the $D$ - and $F$-waves, the partial-wave amplitudes for three out of the four possible channels could be resolved from the data in the analysis of Ref. 14. These triplets of amplitudes are predicted to obey the relations:

$$
D D_{33}=\frac{4}{5 \sqrt{10}} \cdot D D_{13}+\frac{9}{10} \sqrt{\frac{7}{5}} \cdot D D_{15}
$$

and

$$
F F_{37}=\frac{7}{6 \sqrt{6}} \cdot F F_{15}+\frac{2}{3} \sqrt{\frac{5}{3}} \cdot F F_{35} .
$$

In Fig. 11 we have displayed the experimental $D D_{33}$ and $F F_{37}$ amplitudes (indicated by solid lines) juxtaposed with the appropriate linear combinations dic-

data, but one should note that there are some large deviations, for example, in the $S D_{31}$ and $P P_{33}$ partial waves.

$\dagger$ The notation is $L L_{2 I, 2 J}^{\prime}$, with $L$ and $L^{\prime}$ the incoming and outgoing pion angular momenta, respectively. 

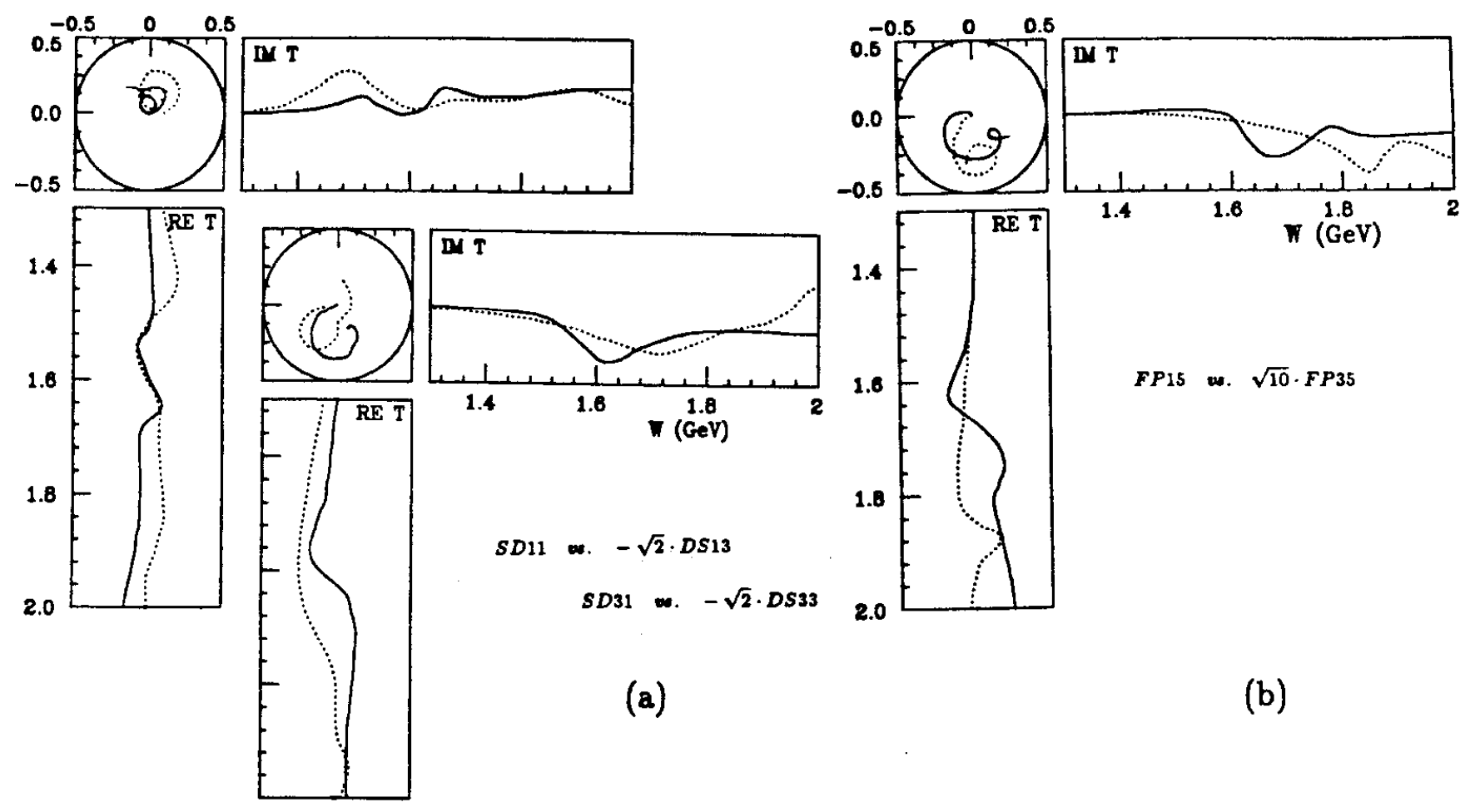

(b)

Fig. 10. $\pi N \rightarrow \pi \Delta$ processes in which the pion jumpe two units of angular momentum: (a). Test of Eq. (II.24a) by comparison of the various $S D$ and $D S \pi N \rightarrow \pi \Delta$ partial-wave amplitudes. The upper graph plots $S D_{11}$ against $-\sqrt{2} \cdot D S_{13}$; the lower graph plots $S D_{31}$ against $-\sqrt{2} \cdot D S_{33}$. (b). Test of Eq. (II.24b) by comparison of $F P_{18}$ to $-\sqrt{10} \cdot F P_{35}$. In each case, the first-named amplitude is represented by the solid curve. Here and in Figs. 11 and 12, we have used the values of these amplitudes corresponding to the fits presented by Ref. 14 .

tated by (II.25) (dotted lines). Although in the first instance (where again there is mixing with the translational mode) the shape of the Argand plot is reasonably rendered, the predicted curve is obviously too big by roughly a factor of four. In the second case, however, as for $\boldsymbol{F}$-waves in general, the agreement is quite respectable.

Unfortunately, out of the four possible $P P$ processes, only $P P_{11}$ and $P P_{33}$ were considered by Manley, et al., to be adequately determined by the data. This makes it impossible for us to test the validity of Eq. (II.19) for this case. 


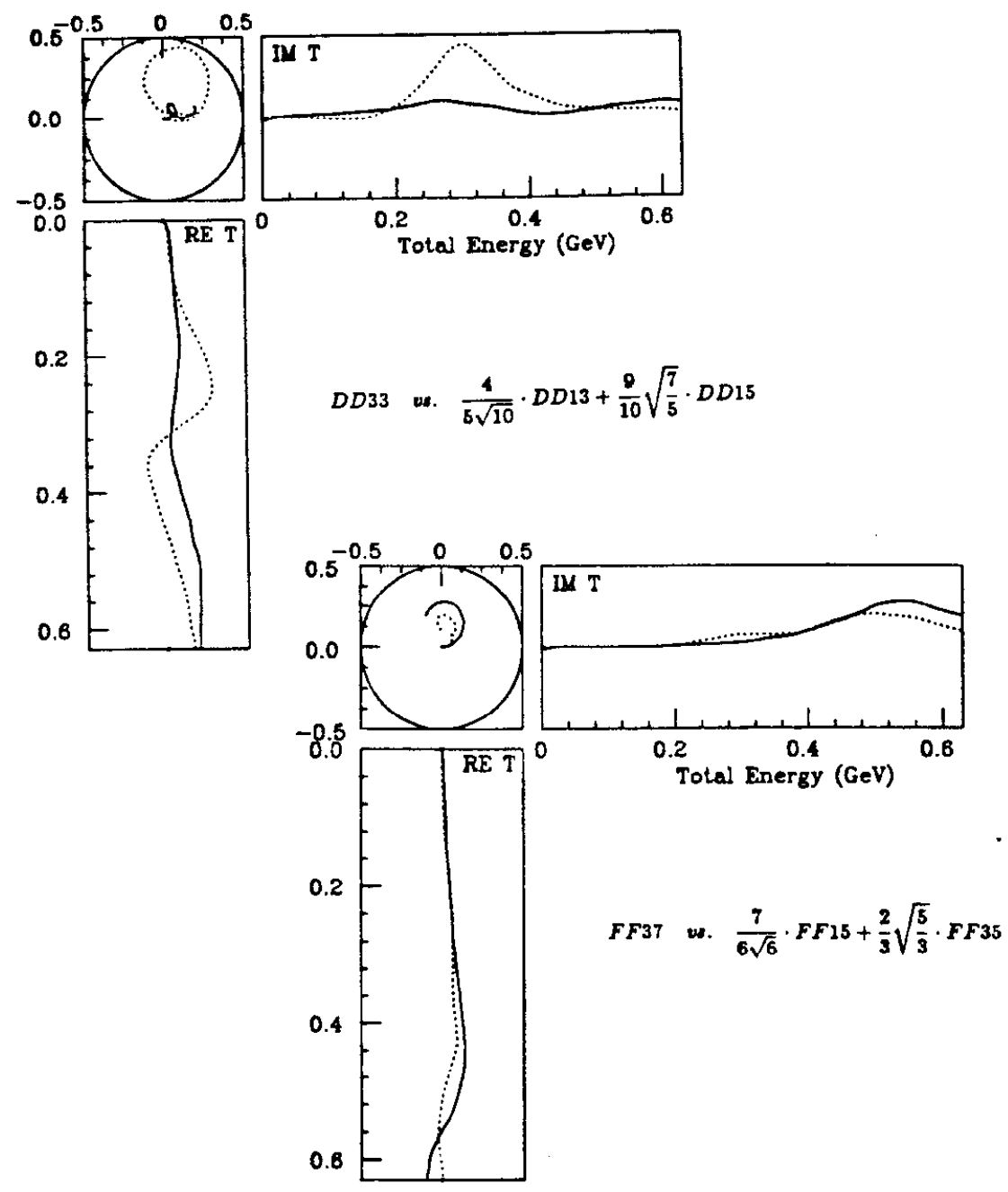

Fig. 11. $\pi N \rightarrow \pi \Delta$ processes in which the initial and final pion angular momenta are equal. The $D D_{33}$ and $F F_{37}$ amplitudes (solid lines) are juxtaposed with the linear combinations to which they are predicted to correspond via Eq. (II.25) (dotted lines).

If, however, we assume the $P P_{13}$ amplitude to be small, Eq. (II.19)b suggests that the $P P_{11}$ and $P P_{33}$ amplitudes will have the same sign; this is indeed what is observed experimentally.

We turn, finally, to Eq. (II.21), which links the processes $\pi N \rightarrow \pi N$ and 

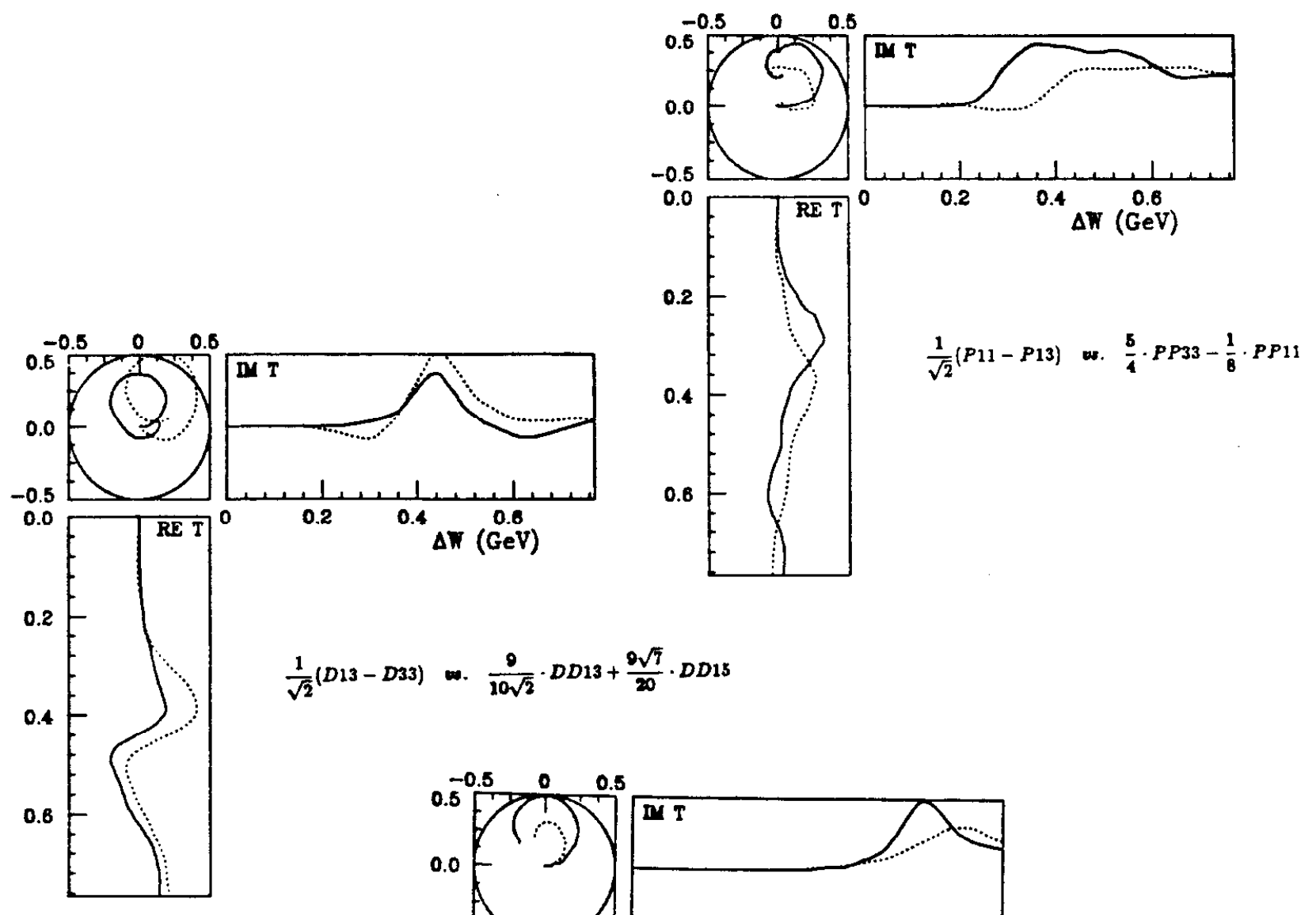

$$
\frac{1}{\sqrt{2}}(D 13-D 33) \text { w. } \frac{9}{10 \sqrt{2}} \cdot D D 13+\frac{9 \sqrt{7}}{20} \cdot D D 13
$$

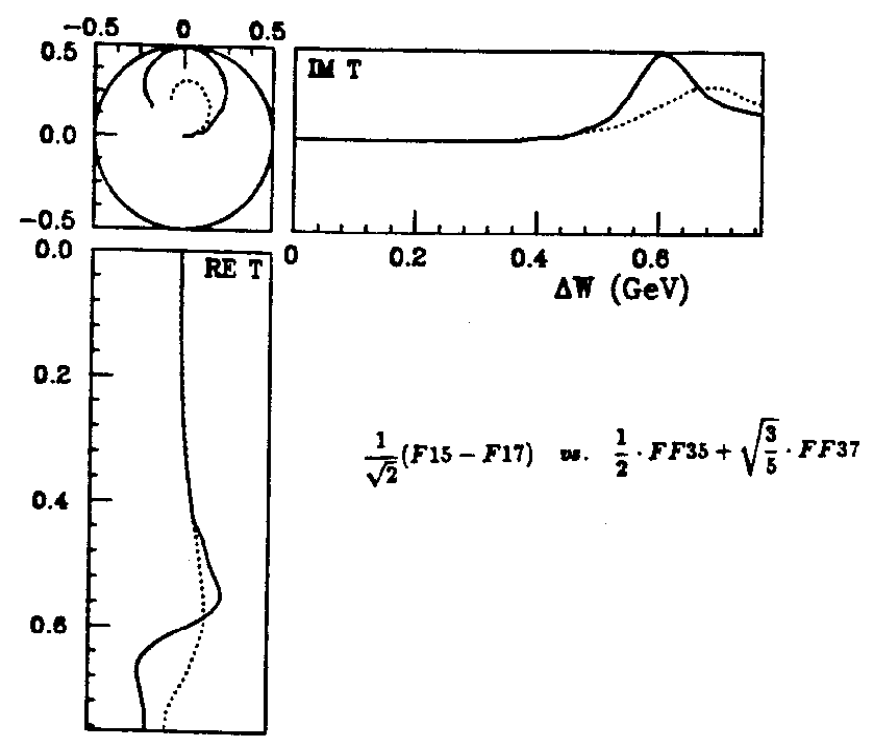

Fig. 12. Comparison of $\pi N \rightarrow \pi N$ and $\pi N \rightarrow \pi \Delta$ scattering in the same partial wave. The linear combinations of elastic amplitudes (solid lines) are juxtaposed with the appropriate combinations of inelastic amplitudes (dotted lines) as dictated by Eq. (II.26). Excitation energy $\Delta W$ is measured from the $\pi N$ threshold in the elastic case, and from the average of the $\pi N$ and $\pi \Delta$ threshold in the inelastic case.

$\pi N \rightarrow \pi \Delta$. The relations which follow from (II.21) may be expressed in several different ways, by combining this equation with the relations (II.18) and (II.19) already discussed. For example, one may obtain: 


$$
\begin{gathered}
\frac{1}{\sqrt{2}}\left(P_{11}-P_{13}\right)=\frac{5}{4} \cdot P P_{33}-\frac{1}{8} \cdot P P_{11} \\
\frac{1}{\sqrt{2}}\left(D_{13}-D_{33}\right)=\frac{9}{10 \sqrt{2}} \cdot D D_{13}+\frac{9 \sqrt{7}}{20} \cdot D D_{15} \\
\frac{1}{\sqrt{2}}\left(F_{15}-F_{17}\right)=\frac{1}{2} \cdot F F_{35}+\sqrt{\frac{3}{5}} \cdot F F_{37} .
\end{gathered}
$$

The left- and right-hand sides of these equations are compared in Fig. 12; they are indicated by solid and dotted lines, respectively. The comparisons are typical among the choices which we have examined, though different choices yield curves of very different size. ${ }^{\star}$ In making this comparison, it is also necessary to choose a convention for relating energies in $\pi N \rightarrow \pi \Delta$ to those in $\pi N \rightarrow \pi N$. It is not clear to us to us whether it is best to define the excitation energy in $\pi N \rightarrow \pi \Delta$ as starting from the $\pi N$ or the $\pi \Delta$ threshold; as a compromise we have taken the average of the two (for these graphs only). Once again, although the sizes of the amplitudes are not in especially close agreement, the signs are correctly given and the general shapes are similar.

All in all, we can conclude that the limited $\pi N \rightarrow \pi \Delta$ data, while not particularly compelling in and of itself, is certainly consistent with the elegant interpretation of the baryon as a soliton in the field of pions.

* We should note, though, that in (II.26) we have avoided combinations which require cancelations among large amplitudes, or which involve the exceptional channels $P_{39}$ and $D_{35}$. 


\section{THE BARYON SPECTRUM OF THE SKYRME MODEL}

In this chapter we shall apply the formalism developed in Chapter II to the particular case of the Skyrme model..$^{[5]}$ A pleasing feature of this model is that one can readily calculate many static properties of baryons such as magnetic moments and charge-radii. ${ }^{[13]}$ With the glaring exception of $g_{A}$, these typically agree with experiment to within $30 \%$, when the adjustable parameters of the model are chosen to give the nucleon and delta masses correctly.

Much less attention, however, has been focused on the dynamical properties of skyrmions. Important progress in this direction was made independently by Zahed, Meissner and Kaulfuss ${ }^{[20]}$ and Breit and Nappi. ${ }^{[2]}$ Interpreting fluctuations around the soliton as pion-nucleon scattering, these authors calculated the phase-shifts in the "breathing-mode" of the skyrmion and looked for a resonance in this channel by seeing if and when the phase-shift crossed $90^{\circ}$. (We shall adopt a different criterion for the existence of a resonance below.) With this criterion there is no resonance for the case of massless pions, and a marginal resonance at $1270 \mathrm{MeV}$ for massive pions which Breit and Nappi tentatively identified with the real-world Roper resonance $P_{11}(1440) .{ }^{\dagger}$ We shall see that this identification needs to be modified.

In this spirit we shall examine the process $\pi N \rightarrow \pi N$ in all channels of isospin and angular momentum for which experimental data was available for comparison. As in the previous chapter, we will restrict ourselves to a lowestorder calculation in $1 / N$.

We are not motivated by the belief that there is anything especially fundamental about the Skyrme Lagrangian. Rather, we find it instructive to see how well the actual spectrum of nucleon and delta resonances can be fit starting

$\dagger$ We shall follow the standard notation $L_{2 I, 2 J}$ for resonances, where $L=S, P, D, F \ldots$ denotes the partial wave in which the resonance is formed, and $I$ and $J$ give the total isospin and angular momentum. Nucleon and delta resonances are characterised by $I=1 / 2$ and $I=3 / 2$, respectively. 
from a model that contains no explicit baryon fields and only three adjustable parameters: $\left\{f_{\pi}, e, m_{\pi}\right\}$. Indeed, we shall specialize to a two-parameter fit (oneparameter if the proton mass is fixed) by working in the chiral limit $m_{\pi}=0$. Nevertheless our findings are in generally good agreement with the real world for energies up to $3 \mathrm{GeV}$, with masses predicted on the average to within $8 \%$ of their actual values. (Our baryon-mass predictions are presented in Table I of Section III.2.) This is all the more surprising given the rather drastic nature of our approximations, such as completely neglecting baryon recoil (cf. Section II.1). Another noteworthy result of the Skyrme model is that it correctly reproduces the big-small-small-big pattern found in Nature, as discussed in Chapter II. And finally, as a bonus, we shall find that our 'best-fit' values for the Skyrme parameters substantially improve some of the static properties of the model as calculated by Adkins, Nappi and Witten. ${ }^{[23]}$

We should at the outset mention some of our disappointments as well. The most obvious of these is our failure to find in pion-skyrmion scattering what in the real world is the most spectacular baryon resonance of all, the delta itself; likewise the $P_{11}$ and $S_{31}$ channels at low energies are manifestly in poor agreement with experiment. It is not clear to us whether these represent failures of the Skyrme model or merely of our approximations near threshold. Either way, we shall argue that these discrepancies are not necessarily fatal to the model by showing that small perturbations can easily restore the correct low-energy behavior in these channels. In particular we can expect the delta to reappear in the next order in $1 / N$. One can even take the optimistic view that these chiral soliton models provide precisely the right framework for understanding why some of the $S-, P$ - and $D$-wave channels contain clear, low-lying resonances, while others, in stark contrast, are marked by repulsive behavior near threshold. We will return to a full discussion of these matters in Sec. III.2, where we take up pion-nucleon scattering, but first, in Sec. III.1, we lay the groundwork by examining the "reduced" processes in which a pion scatters elastically off an unrotated skyrmion (recall that nucleons and deltas should properly be identified 
with rotating solitons).

Much of the development of this chapter is similar to work carried out at Siegen University. ${ }^{[22,18]}$ In particular, the Argand plots presented in Ref. 18 (for $F$-wave $\pi N$ scattering only) appear to be in good numerical agreement with our own.

\section{Pion-Scattering from Unrotated Skyrmions}

The Skyrme Lagrangian with a chiral-symmetry-breaking mass-term is given by

$$
\mathcal{L}=\frac{f_{\pi}^{2}}{16} \operatorname{Tr} \partial_{\mu} U \partial_{\nu} U^{\dagger}+\frac{1}{32 e^{2}} \operatorname{Tr}\left[\left(\partial_{\mu} U\right) U^{\dagger},\left(\partial_{\nu} U\right) U^{\dagger}\right]^{2}+\frac{f_{\pi}^{2} m_{\pi}^{2}}{8}(\operatorname{Tr} U-2) .
$$

Here $f_{\pi}$ is the pion decay constant (186 MeV in the real world), $m_{\pi}$ is the pion mass, and $e$ is a new, dimensionless coupling constant peculiar to the model. The "small parameter" $1 / N$ enters the Lagrangian through $f_{\pi}$ and $e$, which behave like $N^{\frac{1}{2}}$ and $N^{-\frac{1}{2}}$ in the the large- $N$ limit, respectively.

As before, we shall focus on the hedgehog solution

$$
U_{0}=e^{i F(r) \widehat{\mathbf{r}} \cdot \vec{\sigma}} .
$$

Indeed, if we plug this ansatz into (III.1) and look at small fluctuations about the soliton

$$
F(r) \widehat{\mathbf{r}} \longrightarrow F(r) \widehat{\mathbf{r}}+\frac{2}{f_{\pi}} \vec{\pi}(\vec{x}, t)
$$

we obtain the Euler equation

$$
\begin{aligned}
(\hat{\mathbf{r}} \cdot \vec{\pi})\left[\left(\tilde{r}^{2}+8 \sin ^{2} F\right) F^{\prime \prime}+2 \tilde{r} F^{\prime}+4 \sin 2 F\left(F^{\prime}\right)^{2}\right. \\
\\
\left.\quad-\sin 2 F-\frac{4}{\widetilde{r}^{2}} \sin ^{2} F \sin 2 F-\tilde{m}_{\pi}^{2} \widetilde{r}^{2} \sin F\right]=0
\end{aligned}
$$

where the derivatives are taken with respect to the dimensionless variable $\tilde{r}=$ $e f_{\pi} r$, and $\tilde{m}_{\pi}=m_{\pi} / e f_{\pi}$. Field configurations of the form (III.2) are thus automatically stable against angular fluctuations $\vec{\pi}=a \hat{\theta}+b \hat{\phi}$. To render them 
stable against radial fluctuations as well, one requires the expression in square brackets to vanish, which gives the defining equation for $F(r)$. Straightforward Hamiltonian quantum mechanics in the collective coordinates $A$ then yields for the nucleon and delta masses ${ }^{[18]}$

$$
m_{N}=m_{0}+\gamma e^{3} f_{\pi}\left(\frac{1}{2} \times \frac{3}{2}\right) ; \quad m_{\Delta}=m_{0}+\gamma e^{3} f_{\pi}\left(\frac{3}{2} \times \frac{5}{2}\right)
$$

where $m_{0}$ is the mass of the unrotated skyrmion (approximately $36.5 f_{x} / e$ ) and $\gamma \approx 4.7 \times 10^{-3}$.

This concludes our brief review of the Skyrme model; in the remainder of this section we put aside the issue of collective coordinates and focus purely on the question of pion-scattering from unrotated skyrmions of the form (III.2). Explicit forms of the rather unwieldy differential operators involved are presented in Appendix C. The results for the $S$-matrix that we obtain in this section will be reassembled in the next to yield the amplitudes for the physical processes $\pi N \rightarrow \pi N$.

Following Chapter II, we incorporate pions into the Skyrme Lagrangian (III.1) by substituting (III.3) into the exponent of (III.2). After integration by parts one obtains

$$
S=-\int d t m_{0}+\int d^{4} x \pi^{i *}(\vec{x}, t) \widehat{L}_{i j} \pi^{j}(\vec{x}, t)+O\left(\pi^{3} / f_{\pi}\right)
$$

with $\widehat{L}$ a complicated $3 \times 3$ matrix of second-order differential operators. (We are allowing complex pion fields as a convenience; this way we are spared from having to take real parts of spherical harmonics and of $e^{i \omega t}$ throughout.) Consistent with the large- $N$ spirit that motivates the model we will henceforth drop all terms of cubic or higher order in the pion fields; these are damped by powers of $f_{\pi} \sim N^{\frac{1}{2}}$. As a result, the equations of motion for the pion fields that we will derive will be linear ones. 
We can make substantial progress by realizing that $\widehat{L}$, complicated though it may be, preserves the symmetry $\mathbf{K}=\mathbf{I}$ (pion) $+\mathbf{L}$ (pion). Explicitly,

$$
\left(-i \epsilon_{i j k} r^{i} \partial_{j} \delta_{b c}+i \epsilon_{k b c}\right) \widehat{L}_{b a}-\widehat{L}_{c b}\left(-i \epsilon_{i j k} r^{i} \partial_{j} \delta_{b a}-i \epsilon_{k b a}\right)=0
$$

Following Appendix A, we can take advantage of this fact by expanding the pion field in terms of the vector spherical harmonics

$$
\Pi_{L}^{K K_{z}}=\left(\begin{array}{c}
\left\langle L 1 K_{z}-1,1 \mid K, K_{z}\right\rangle Y_{L, K_{z}-1}(\Omega) \\
\left\langle L 1 K_{z} 0 \mid K, K_{z}\right\rangle Y_{L, K_{z}}(\Omega) \\
\left\langle L 1 K_{z}+1,-1 \mid K, K_{z}\right\rangle Y_{L, K_{z}+1}(\Omega)
\end{array}\right)
$$

which are states of definite $K^{2}$ and $K_{z}$. Accordingly we plug

$$
\begin{aligned}
\vec{\pi}(\vec{x}, t)=\sum_{K, K_{z}}\left\{\psi_{-}^{K K_{z}}(r, t) \Pi_{K-1}^{K K_{z}}(\Omega)\right. & +\psi_{0}^{K K_{z}}(r, t) \Pi_{K}^{K K_{x}}(\Omega) \\
& \left.+\psi_{+}^{K K_{x}}(r, t) \Pi_{K+1}^{K K_{x}}(\Omega)\right\}
\end{aligned}
$$

into (III.6). Parity precludes the $\psi_{0}$ 's from mixing with the $\psi_{ \pm}$'s; $\psi_{+}$and $\psi_{-}$can mix in this model, however, as they do in Nature, where jumps of two units of pion angular momentum are allowed in the process $\pi N \rightarrow \pi \Delta$.

The angular integration can be performed, thanks to the identities

$$
\begin{aligned}
& \nabla \cdot \Pi_{K-1}^{K K_{z}}=-\frac{K-1}{r} \widehat{\mathbf{r}} \cdot \Pi_{K-1}^{K K_{z}}=-\frac{K-1}{r} \sqrt{\frac{K}{2 K+1}} Y_{K K_{x}} \\
& \nabla \cdot \Pi_{K+1}^{K K_{z}}=\frac{K+2}{r} \hat{\mathbf{r}} \cdot \Pi_{K+1}^{K K_{z}}=-\frac{K+2}{r} \sqrt{\frac{K+1}{2 K+1}} Y_{K K_{z}} \\
& \nabla \cdot \Pi_{K}^{K K_{z}}=\hat{\mathbf{r}} \cdot \Pi_{K}^{K K_{z}}=0
\end{aligned}
$$


We are left with

$$
\begin{aligned}
S & =-\int d t m_{0}+\sum_{K, K_{z}} \int r^{2} d r \psi_{0}^{K K_{z} *}(r, t) \widehat{L}_{0}^{K} \psi_{0}^{K K_{z}}(r, t) \\
& +\sum_{K, K_{z}} \int r^{2} d r\left(\psi_{-}^{K K_{z} *}(r, t), \psi_{+}^{K K_{z}^{*}}(r, t)\right)\left(\begin{array}{ll}
\widehat{L}_{-}^{K} & \widehat{L}_{-+}^{K} \\
\hat{L}_{+-}^{K} & \widehat{L}_{++}^{K}
\end{array}\right)\left(\begin{array}{l}
\psi_{-}^{K K_{x}}(r, t) \\
\psi_{+}^{K K_{*}}(r, t)
\end{array}\right)
\end{aligned}
$$

where the $\widehat{L}^{K}$ 's are complicated second-order differential operators in $r$ and $t$ alone. We will refer to the $2 \times 2$ matrix of operators here as $\widehat{\mathbf{L}}^{K}$ and the 2component column-vector of wavefunctions as $\Psi^{K}$.

The determination of phase-shifts now proceeds in a completely straightforward manner, exactly as in Ref. 21. The "normal-mode" equations to be solved are

$$
\widehat{L}_{0}^{K}\left[\psi_{0}^{K}(r) e^{i \omega t}\right]=0
$$

and

$$
\widehat{\mathbf{L}}^{K}\left[\Psi^{K}(r) e^{i \omega t}\right]=0
$$

for all $\omega$; here we are assuming that $\widehat{L}$ and $\widehat{\mathbf{L}}$ have been chosen with care to be self-adjoint. By time-reversal invariance $\widehat{L}$ and $\hat{\mathbf{L}}$ are real operators, so it suffices to consider the real radial wavefunctions that are regular (i.e., square-integrable) at the origin and integrate out past the point where the skyrmion profile $F(r)$ is negligible. In this regime the theory is one of free pions, so $\psi_{0}^{K}$ can be fit to $A(\omega) j_{K}(k r)+B(\omega) n_{K}(k r)$ with $k=\sqrt{\omega^{2}-m_{\pi}^{2}}$. (We follow Messiah ${ }^{[25]}$ in our definitions of the spherical Bessel functions.) The $S$-matrix in this channel is extracted by rewriting this as

$$
\text { constant } \times\left(h_{K}^{-}-s_{K K K}(\omega) h_{K}^{+}\right)
$$

yielding

$$
\mathbf{8}_{K K K}(\omega)=-(B+i A)^{-1}(B-i A)
$$


which lies on the unit circle. (Following Chapter II, we shall represent the reduced amplitudes by $8_{K L^{\prime} L}$, where $L$ and $L^{\prime}$ refer to the incoming and outgoing angular momentum of the pion, respectively.)

The ' $2 \times 2$ ' case (III.12b) proceeds analogously. Near the origin for each $K \geq 1$ there are two independent regular solutions $\Psi_{1}^{K}$ and $\Psi_{2}^{K}$, which behave asymptotically as

$$
\Psi_{i}^{K} \sim\left(\begin{array}{l}
A_{i}(\omega) j_{K-1}(k r)+B_{i}(\omega) n_{K-1}(k r) \\
C_{i}(\omega) j_{K+1}(k r)+D_{i}(\omega) n_{K+1}(k r)
\end{array}\right) \quad i=1,2
$$

(The exception is the translational zero-mode (III.19b) below; the second zeroenergy solution which is well-behaved at the origin blows up for large $r$.) If we work in the convenient basis in which the incoming pions are in pure $(K-1)$ waves or $(K+1)$-waves of orbital angular momentum, the $2 \times 2 S$-matrices are given by

$$
\begin{aligned}
\mathbf{S}_{K} & =\left(\begin{array}{ll}
\mathbf{s}_{K, K-1, K-1} & \mathbf{s}_{K, K-1, K+1} \\
\mathbf{s}_{K, K+1, K-1} & \mathbf{s}_{K, K+1, K+1}
\end{array}\right) \\
& =-\left(\begin{array}{ll}
B_{1}+i A_{1} & D_{1}+i C_{1} \\
B_{2}+i A_{2} & D_{2}+i C_{2}
\end{array}\right)^{-1}\left(\begin{array}{ll}
B_{1}-i A_{1} & D_{1}-i C_{1} \\
B_{2}-i A_{2} & D_{2}-i C_{2}
\end{array}\right)
\end{aligned}
$$

Note that $\mathbf{S}_{K}$ as given is correctly invariant under different choices of regular solutions $\Psi_{1}^{\prime}=\alpha \Psi_{1}+\beta \Psi_{2}$ and $\Psi_{2}^{\prime}=\gamma \Psi_{1}+\delta \Psi_{2}$. Furthermore it is trivial to prove that a matrix of the form $-M^{-1} M^{*}$ can be unitary if and only if it is also complex-symmetric, so that $\mathbf{s}_{213}=\mathbf{s}_{231}$, etc. This result, which follows generally from time-reversal invariance, ${ }^{[23]}$ provides a useful check on one's numerical calculations. Accordingly we can parametrize $S_{K}$ as

$$
\left(\begin{array}{cc}
\eta_{K}^{D} e^{2 i \delta_{K, K-1, K-1}} & \eta_{K}^{O D} e^{2 i \delta_{K, K+1, K-1}} \\
\eta_{K}^{O D} e^{2 i \delta_{K, K+1, K-1}} & \eta_{K}^{D} e^{2 i \delta_{K, K+1, K+1}}
\end{array}\right)
$$

where the phase-shifts and absorption parameters are constrained by unitarity 
to obey

$$
\delta_{K, K-1, K-1}(\omega)+\delta_{K, K+1, K+1}(\omega)-2 \delta_{K, K+1, K-1}(\omega)=\left(n+\frac{1}{2}\right) \pi
$$

and

$$
\left(\eta_{K}^{D}(\omega)\right)^{2}+\left(\eta_{K}^{O D}(\omega)\right)^{2}=1
$$

(The superscripts $D$ and $O D$ here stand for diagonal and off-diagonal.)

We should mention the special case $K=0$, for which only the right-most term in (III.9) exists; this is the breathing-mode $\vec{\pi} \propto \widehat{r}$. In our notation the only nonvanishing component of the $S$-matrix when $K=0$ is $s_{011}=e^{2 i \delta_{011}}$. We further note that the zero-modes corresponding to rotations and translations of the skyrmion,

$$
\Pi_{1}^{1 m}(\Omega) F(r)
$$

and

$$
\left(F^{\prime}+\frac{2 F}{r}\right) \Pi_{0}^{1 m}(\Omega)+\sqrt{2}\left(\frac{F}{r}-F^{\prime}\right) \Pi_{2}^{1 m}(\Omega)
$$

respectively, appear in the model as threshold bound-states. These will play a crucial role in our later discussion.

Some further details of the above procedure, including explicit expressions for $\widehat{L}^{K}$ and $\widehat{\mathbf{L}}^{K}$, are given in App. C. The results of our phase-shift analysis for the various $S$-matrix components $\mathrm{s}_{K L L^{\prime}}$ with $L, L^{\prime} \leq 7$ are plotted in Figures 13-18. We have restricted our numerical analysis here to the case $m_{\pi}=0$.

Fig. 13 depicts the phase-shifts $\delta_{K K K}$ graphed against pion-energy $\omega$, measured in units of $e f_{\pi}$. (This number should be thought of as lying somewhere between 700 and $900 \mathrm{MeV}$; we will take up this matter in Sec. III.2.) The absence of a resonance for $K=1$ is of course due to the presence in this channel of the rotational zero-mode (III.19a). For $K>1$ the obvious trend is for the resonances to become broader and more massive with increasing $K$. 


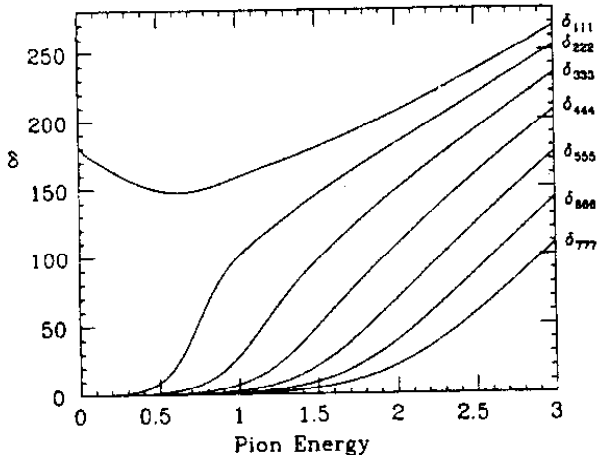

Fig. 13. Phase shifts $\delta_{K K K}$ plotted vs pion energy $\omega$, measured in units of $e f$.

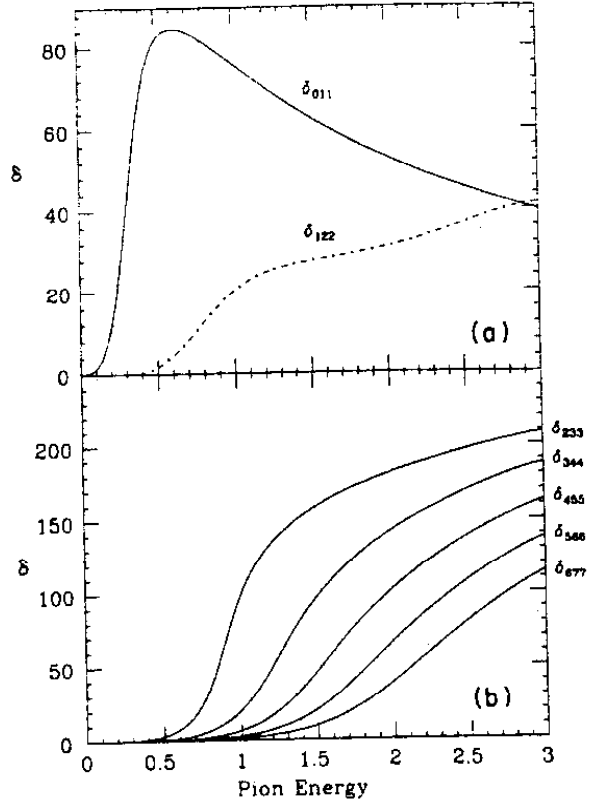

Fig. 14. Phase shifts $\delta_{K, K+1, K+1}$ plotted vs pion energy $\omega$, measured in units of $e f_{\pi}$.

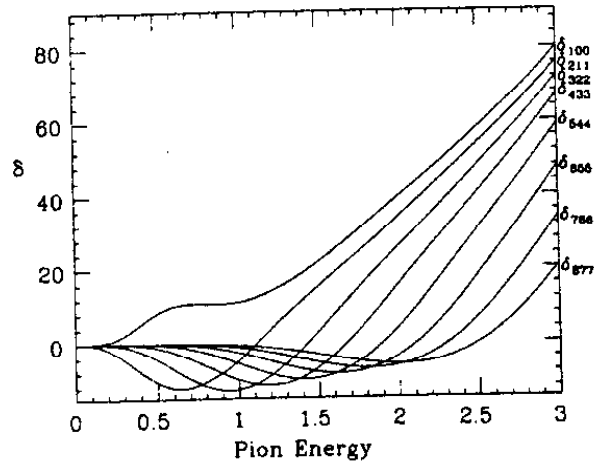

Fig. 15. Phase shifts $\delta_{K, K-1, K-1}$ plotted vs pion energy $\omega$, measured in units of $e f_{*}$.

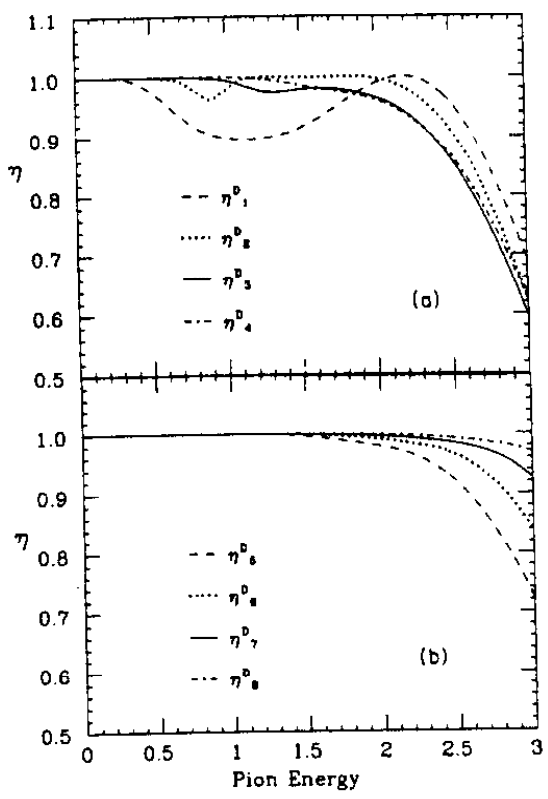

Fig. 16. Absorption parameters $\eta_{K}^{\mathrm{D}}$ plotted vs pion energy $\omega$, measured in units of $e f_{\pi}$.

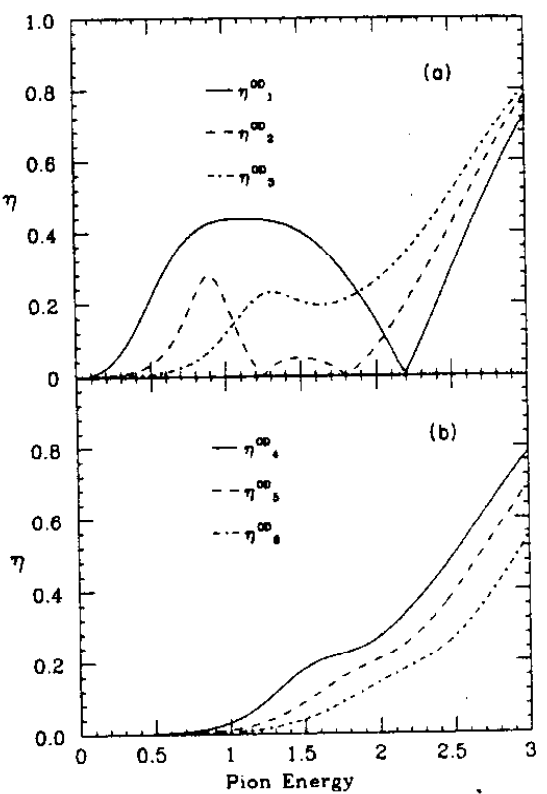

Fig. 17. Absorption parameters $\eta_{K}^{\mathrm{OD}}$ plotted vs pion eñergy $\omega$, measured in units of $e f_{\mathbf{x}}$.

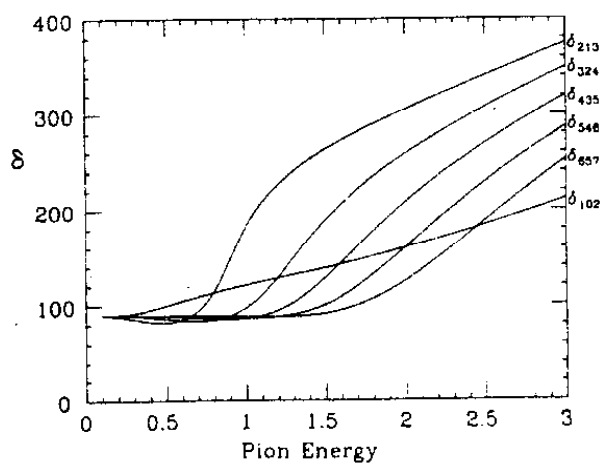

Fig. 18. Phase shifts $\delta_{K, K-1, K+1}$ plotted vs pion energy $\omega$, measured in units of $e f_{\mathrm{m}}$. 
Figures 14, 15 and 16 present our results for the diagonal components of $\mathbf{S}_{K}$. Clearly, for $L \geq 3$, the phase-shifts $\delta_{L-1, L, L}$ rise sooner than $\delta_{L L L}$, and certainly much more dramatically than $\delta_{L+1, L, L}$. As a consequence, the location of the resonances in the corresponding channels of $\pi N$ scattering can essentially be read off from Fig. 14b. In contrast to $L \geq 3$ note the tepid behavior of $\delta_{011}$ and $\delta_{122}$ (Fig. 14a). The former is the breathing-mode; as for the latter, the weak rise, like that of $\delta_{111}$, is due to mixing with the translational mode (III.19b).

Figures 17 and 18 present the corresponding graphs for the off-diagonal elements $\mathbf{s}_{K, K+1, K-1}$. These describe processes in which the orbital angular momentum of the pion jumps by two units. By conservation of angular momentum these processes are $\pi N \rightarrow \pi \Delta$ necessarily, and so we defer discussion to the future.

At this point we should make a technical comment about how we determined the location of resonances. In principle it is unsatisfactory merely to find where the phase-shift crosses $90^{\circ}$, due to the often significant effects of background. A much more reliable criterion is to look for a well-defined peak in the speed $|d S / d \omega|$ in those regions where the amplitude is curving counter-clockwise in the Argand diagram. Surprisingly, for $\mathbf{s}_{K, K+1, K+1}$ this occurs when the phase-shifts are approximately $45^{\circ}$. We turn now to our main topic of pion-nucleon scattering in the Skyrme model.

\section{Pion-Nucleon Scattering}

So far we have discussed the (linearized) equations of motion for pions moving in a fixed external skyrmion background. To relate this to $\pi N$ scattering requires the use of Eq. (II.11). We find:

$$
\begin{aligned}
& \mathbf{S}_{L \frac{1}{2}, L-\frac{1}{2}}=\frac{2 L-1}{3 L} \mathrm{~s}_{L-1, L, L}+\frac{L+1}{3 L} \mathbf{s}_{L L L} \\
& \mathbf{S}_{L \frac{1}{2}, L+\frac{1}{2}}=\frac{L}{3 L+3} \mathrm{~s}_{L L L}+\frac{2 L+3}{3 L+3} \mathrm{~s}_{L+1, L, L}
\end{aligned}
$$

